\title{
Derived functor modules arising as large irreducible constituents of degenerate principal series
}

\author{
Hisayosi Matumoto and Peter E. Trapa
}

\begin{abstract}
For the groups $G=\mathrm{Sp}(p, q), \mathrm{SO}^{*}(2 n)$, and $\mathrm{U}(m, n)$, we consider degenerate principal series whose infinitesimal character coincides with a finite-dimensional representation of $G$. We prove that each irreducible constituent of maximal Gelfand-Kirillov dimension is a derived functor module. We also show that at an appropriate 'most singular' parameter, each irreducible constituent is weakly unipotent and unitarizable. Conversely we show that any weakly unipotent representation associated to a real form of the corresponding Richardson orbit is unique up to isomorphism and can be embedded into a degenerate principal series at the most singular integral parameter (apart from a handful of very even cases in type D). We also discuss edge-of-wedge-type embeddings of derived functor modules into degenerate principal series.
\end{abstract}

\section{Introduction}

In the representation theory of real reductive Lie groups, there are two fundamental constructions of representations: parabolic induction and cohomological induction. For the purposes of this introduction, we call a representation parabolically induced (respectively, cohomologically induced) from a one-dimensional representation a degenerate principal series (respectively, derived functor module). Each construction has a natural geometric interpretation and the geometry involved often suggests a relationship between the two kinds of representations. The situation can become quite complicated (in the case of a split group such as $\operatorname{Sp}(2 n, \mathbb{R})$, for instance) and it is difficult to extract simple, clean statements. However, for groups whose Cartan subgroups are always connected, the situation turns out to be rather more simple. In this article, we study the relation between degenerate principal series and derived functor modules for such groups. Our first main theorem is as follows. (We call a weight $G$-integral if it appears as a weight of a finite-dimensional representation of $G$.)

Theorem A (Corollary 6.13, Theorem 7.3). Let $G$ be $\operatorname{Sp}(p, q), \mathrm{SO}^{*}(2 n)$, or $\mathrm{U}(m, n)$. Let $P$ be a parabolic subgroup of $G$ and let $X$ be a representation of $G$ parabolically induced from a onedimensional representation of $P$. Assume that $X$ has a $G$-integral infinitesimal character. Then any irreducible constituent $V$ of $X$ of maximal Gelfand-Kirillov dimension is a derived functor module in the weakly fair range (in the sense of [ $\operatorname{Vog} 88]$ ). Moreover, the multiplicity of $V$ in $X$ is one.

Among the degenerate principal series representations induced from $P$, those with the smallest possible integral infinitesimal character (among all degenerate principal series induced from $P$ ) are particularly interesting, and so we turn our attention to them. We call such representations integrally weakly unipotent degenerate principal series. This is a completely naive definition, but

Received 11 February 2005, accepted in final form 16 June 2006.

2000 Mathematics Subject Classification 22E46, $22 \mathrm{E} 47$.

Keywords: unitary representation, unipotent representation, parabolic induction, derived functor module.

This journal is (c) Foundation Compositio Mathematica 2007. 


\section{LARGE IRREDUCIBLE CONSTITUENTS OF DEGENERATE PRINCIPAL SERIES}

the terminology is potentially dangerous since the more sophisticated notion of weakly unipotent representations has already been defined in [Vog87] (see Definition 5.1 below). According to the results below, the definitions are consonant.

For $G=\mathrm{U}(m, n)$, an integrally weakly unipotent degenerate principal series representation is unitarily induced and its precise structure is known from [Mat96] and [Tra01]. On the other hand, for $G=\operatorname{Sp}(p, q)$ and $\mathrm{SO}^{*}(2 n)$, the situation is a little more complicated. Such representations are not unitarily induced, for instance. Moreover, an integrally weakly unipotent degenerate principal series representation for a given parabolic subgroup need not be unique. However, the distribution characters of two such representations coincide, and thus they have the same composition factors. We have the following result.

Theorem B (Theorem 6.10). Let $G$ be $\operatorname{Sp}(p, q)$ or $\mathrm{SO}^{*}(2 n)$. Let $P$ be a parabolic subgroup of $G$ and let $X$ be an integrally weakly unipotent degenerate principal series representation induced from $P$. Then each irreducible constituent of $X$ is unitarizable. Moreover, if the Richardson orbit corresponding to $P$ is not very even, then each irreducible constituent of $X$ is weakly unipotent in the sense of [ $\operatorname{Vog} 87]$.

Here 'the Richardson orbit corresponding to $P$ ' means the complex nilpotent orbit induced from the zero orbit of the Levi factor of the complexification of the Lie algebra of $P$. In particular, the Richardson orbit corresponding to $P$ is very even if and only if $G=\mathrm{SO}^{*}(4 n)$ and the Levi factor of $P$ is isomorphic to a direct product of general linear groups over the quaternionic field $\mathbb{H}$.

For $G=\operatorname{Sp}(p, q)$ and $\mathrm{SO}^{*}(2 n)$, we characterize the integrally weakly unipotent representations associated to a Richardson orbit corresponding to a parabolic subgroup as follows.

Theorem C (Theorem 6.12). Retain the setting of Theorem B. Let $\mathcal{O}$ denote the complex Richardson orbit associated to $P$. Suppose that $X$ is a weakly unipotent representation attached to $\mathcal{O}$ (Definition 5.1). In addition, suppose that $X$ has integral infinitesimal character. Then $X$ is isomorphic to a derived functor module which arises as an irreducible constituent of an integrally weakly unipotent degenerate principal series representation induced from $P$. In particular, the associated variety of $X$ is the closure of a unique $K_{\mathbb{C}}$ orbit $\mathcal{O}_{K}$ on $\mathcal{O} \cap \mathfrak{s}$ (where $\mathfrak{g}=\mathfrak{k} \oplus \mathfrak{s}$ denotes the complexified Cartan decomposition).

Conversely, for any $K_{\mathbb{C}}$ orbit $\mathcal{O}_{K}$ on $\mathcal{O} \cap \mathfrak{s}$, there exists a unique (up to isomorphism) weakly unipotent representation with an integral infinitesimal character whose associated variety is the closure of $\mathcal{O}_{K}$.

We now turn to the details of the interaction between the geometric interpretations of parabolic and cohomological induction. Let $G$ be a real linear reductive Lie group and let $G_{\mathbb{C}}$ denote its complexification. Write $\mathfrak{g}_{0}$ (respectively, $\mathfrak{g}$ ) for the Lie algebra of $G$ (respectively, $G_{\mathbb{C}}$ ) and let $\sigma$ denote the complex conjugation on $\mathfrak{g}$ with respect to $\mathfrak{g}_{0}$. We fix a maximal compact subgroup $K$ of $G$ and let $\theta$ denote the corresponding Cartan involution. We write $\mathfrak{k}$ for the complexified Lie algebra of $K$.

We fix a parabolic subgroup $P$ of $G$ with a $\theta$-stable Levi part $M$ and nilradical $N$. We let $\mathfrak{p}, \mathfrak{m}$, and $\mathfrak{n}$ denote the complexified Lie algebras of $P, M$, and $N$, respectively, and denote the corresponding analytic subgroups of $G_{\mathbb{C}}$ by $P_{\mathbb{C}}, M_{\mathbb{C}}$, and $N_{\mathbb{C}}$. For $Y \in \mathfrak{m}$, we define

$$
\delta_{P}(Y)=\frac{1}{2} \operatorname{tr}\left(\left.\operatorname{ad}_{\mathfrak{g}}(Y)\right|_{\mathfrak{n}}\right) .
$$

Then $\delta_{P}$ is a one-dimensional representation of $\mathfrak{m}$ and $2 \delta_{P}$ lifts to a holomorphic group homomorphism $\xi_{2 \delta}: M_{\mathbb{C}} \rightarrow \mathbb{C}^{\times}$. Defining $\left.\xi_{2 \delta_{P}}\right|_{N_{\mathbb{C}}}$ to be trivial, we may extend $\xi_{2 \delta_{P}}$ to $P_{\mathbb{C}}$. Let $\mathcal{L}_{\chi}$ be the holomorphic line bundle on $G_{\mathbb{C}} / P_{\mathbb{C}}$ corresponding to a holomorphic character $\chi$ of $M_{\mathbb{C}}$. For a character $\eta: P \rightarrow \mathbb{C}^{\times}$, we consider the unnormalized parabolically induced representation ${ }^{u} \operatorname{Ind}_{P}^{G}(\eta)$. 


\section{H. Matumoto and P. E. Trapa}

Namely, ${ }^{u} \operatorname{Ind}{ }_{P}^{G}(\eta)$ is the $K$-finite part of the space of the $C^{\infty}$-sections of the $G$-homogeneous line bundle on $G / P$ associated to $\eta$. We know that ${ }^{u} \operatorname{Ind}_{P}^{G}(\eta)$ is a Harish-Chandra $(\mathfrak{g}, K)$-module.

If $G / P$ is orientable, then the trivial representation of $G$ is the unique irreducible quotient of ${ }^{u} \operatorname{Ind}_{P}^{G}\left(\xi_{2 \delta}\right)$. If $G / P$ is not orientable, there is a character $\omega$ on $P$ such that $\omega$ is trivial on the identity component of $P$ and the trivial representation of $G$ is the unique irreducible quotient of ${ }^{u} \operatorname{Ind}_{P}^{G}\left(\xi_{2 \delta} \otimes \omega\right)$. This motivates the first of the following definitions.

Definition 1.1 (Definitions 4.2 and 4.6 below). (a) We call a holomorphic character $\chi$ of $M_{\mathbb{C}}$ good if ${ }^{u} \operatorname{Ind}_{P}^{G}\left(\xi_{2 \delta} \otimes \chi\right)$ has a finite-dimensional representation of $G$ as a quotient. In particular, if $G / P$ is orientable, the trivial character is good.

(b) Let $\mathcal{V}$ be an open $G$-orbit on $G_{\mathbb{C}} / P_{\mathbb{C}}$. We say that $\mathcal{V}$ is fine if there is a $\theta$-stable parabolic subalgebra $\mathfrak{q}$ of $\mathfrak{g}$ such that $\mathfrak{q} \in \mathcal{V}$. For example, if $G$ has a compact Cartan subgroup, any open $G$-orbit on $G_{\mathbb{C}} / P_{\mathbb{C}}$ is fine.

(c) For each fine open $G$-orbit $\mathcal{V}$ on $G_{\mathbb{C}} / P_{\mathbb{C}}$, we put

$$
A_{\mathcal{V}}(\chi)=\mathrm{H}^{\operatorname{dim} u \cap \mathfrak{k}}\left(\mathcal{V}, \mathcal{L}_{\chi} \otimes \mathcal{L}_{2 \delta_{P}}\right)_{K \text {-finite }}
$$

From [Won93], $A_{\mathcal{V}}(\chi)$ is a derived functor module [VZ84]. If $\chi$ is good, $A_{\mathcal{V}}(\chi)$ is in the good range in the sense of [Vog88]. We call an open $G$-orbit $\mathcal{V}$ in $G_{\mathbb{C}} / P_{\mathbb{C}}$ good if the Gelfand-Kirillov dimension of $A_{\mathcal{V}}(\mathbb{1})$ is equal to that of ${ }^{u} \operatorname{Ind}_{P}^{G}\left(\xi_{2 \delta}\right)$.

Recall that if $G$ is of Hermitian type and $P$ is a Siegel parabolic subgroup of $G$, there are two open $G$-orbits in $G_{\mathbb{C}} / P_{\mathbb{C}}$ each isomorphic to $G / K$ (the Siegel upper and lower half planes). For such orbits, $A_{\mathcal{V}}(\chi)$ are holomorphic or anti-holomorphic discrete series for a good character $\chi$, and it is well known that we can embed $A_{\mathcal{V}}(\chi)$ into ${ }^{u} \operatorname{Ind}_{P}^{G}\left(\xi_{2 \delta} \otimes \chi\right)$ by taking boundary values at the Shilov boundary.

Our purpose here is to consider other orbits. In this case, the corresponding embedding should be a higher cohomological analog of boundary value maps. We begin with an example.

Example 1.2. Let $G=\mathrm{SO}_{0}(n, 2)$ with $n \geqslant 3$ and let $P$ be a parabolic subgroup whose Levi factor has semisimple part isomorphic to $\mathrm{SO}_{0}(n-1,1)$. For simplicity set $\mathcal{L}=\mathcal{L}_{2 \delta_{P}}$. In this case $X=G_{\mathbb{C}} / P_{\mathbb{C}}$ has three open $G$-orbits. Two of them (say $\mathcal{O}_{+}$and $\mathcal{O}_{-}$) are Hermitian symmetric spaces (symmetric domains of type IV). The remaining open $G$-orbit (say $\mathcal{O}_{0}$ ) is non-Stein and isomorphic to $G /\left(\mathrm{SO}(2) \times \mathrm{SO}_{0}(n-2,2)\right)$. Let $\overline{\mathcal{O}}_{+}$and $\overline{\mathcal{O}}_{-}$be the closures of $\mathcal{O}_{+}$and $\mathcal{O}_{-}$, respectively. In this case, we have $\left(X-\overline{\mathcal{O}}_{+}\right) \cap\left(X-\overline{\mathcal{O}}_{-}\right)=\mathcal{O}_{0}$ and $\left(X-\overline{\mathcal{O}}_{+}\right) \cup\left(X-\overline{\mathcal{O}}_{-}\right)=X-X_{\mathbb{R}}$ where $X_{\mathbb{R}}=G / P$. Hence, we have the following Mayer-Vietoris exact sequence:

$$
H^{2 n-2}\left(X-\overline{\mathcal{O}}_{+}, \mathcal{L}\right) \oplus H^{2 n-2}\left(X-\overline{\mathcal{O}}_{-}, \mathcal{L}\right) \rightarrow H^{2 n-2}\left(\mathcal{O}_{0}, \mathcal{L}\right) \rightarrow H^{2 n-1}\left(X-X_{\mathbb{R}}, \mathcal{L}\right) .
$$

We also have the following exact sequences:

$$
\begin{aligned}
& H^{2 n-2}(X, \mathcal{L}) \rightarrow H^{2 n-2}\left(X-\overline{\mathcal{O}}_{+}, \mathcal{L}\right) \rightarrow H_{\overline{\mathcal{O}}_{+}}^{2 n-1}(X, \mathcal{L}), \\
& H^{2 n-2}(X, \mathcal{L}) \rightarrow H^{2 n-2}\left(X-\overline{\mathcal{O}}_{-}, \mathcal{L}\right) \rightarrow H_{\overline{\mathcal{O}}_{-}}^{2 n-1}(X, \mathcal{L}) .
\end{aligned}
$$

From [Kos61], we have $H^{2 n-2}(X, \mathcal{L})=0$. We can regard $\overline{\mathcal{O}}_{+}$and $\overline{\mathcal{O}}_{-}$as closed convex sets in an open cell of $X$. Hence, from the edge-of-wedge theorem (see [KL83, Théorème 1.1.2]), we have $H_{\overline{\mathcal{O}}_{+}}^{2 n-1}(X, \mathcal{L})=H_{\overline{\mathcal{O}}_{-}}^{2 n-1}(X, \mathcal{L})=0$. Hence, $H^{2 n-2}\left(X-\overline{\mathcal{O}}_{+}, \mathcal{L}\right)=H^{2 n-2}\left(X-\overline{\mathcal{O}}_{-}, \mathcal{L}\right)=0$. We also have the following exact sequence:

$$
0=H^{2 n-1}(X, \mathcal{L}) \rightarrow H^{2 n-1}\left(X-X_{\mathbb{R}}, \mathcal{L}\right) \rightarrow H_{X_{\mathbb{R}}}^{2 n}(x, \mathcal{L}) .
$$

Hence, we have

$$
H^{2 n-2}\left(\mathcal{O}_{0}, \mathcal{L}\right) \hookrightarrow H^{2 n-1}\left(X-X_{\mathbb{R}}, \mathcal{L}\right) \hookrightarrow H_{X_{\mathbb{R}}}^{2 n}(x, \mathcal{L})
$$




\section{LARGE IRREDUCIBLE CONSTITUENTS OF DEGENERATE PRINCIPAL SERIES}

In this case $X_{\mathbb{R}}$ is orientable if and only if $n$ is even. Hence, the local cohomology $H_{X_{\mathbb{R}}}^{2 n}(x, \mathcal{L})$ is the space of hyperfunction sections of the degenerate principal series of $G$ with respect to $\mathcal{L}$ (respectively, $\mathcal{L} \otimes \omega)$ if $n$ is even (respectively, odd). Taking the $K$-finite part, we have

$$
\begin{gathered}
A_{\mathcal{O}_{0}}(\mathbb{1}) \hookrightarrow{ }^{u} \operatorname{Ind}_{P}^{G}\left(\xi_{2 \delta}\right) \quad(\text { if } n \text { is even), } \\
\mathcal{A}_{\mathcal{O}_{0}}(\mathbb{1}) \hookrightarrow{ }^{u} \operatorname{Ind}_{P}^{G}\left(\xi_{2 \delta} \otimes \omega\right) \quad(\text { if } n \text { is odd }) .
\end{gathered}
$$

Such a relatively easy construction of embeddings as in Example 1.2 seems to be difficult to imitate in the general case. However, [Mat88b], [SS90], and [Gin93], etc., suggest some evidence of the existence of the edge-of-wedge embeddings in greater generality. Therefore, we consider the following problem in the setting of Harish-Chandra modules.

Problem D. Let $G$ be a real linear reductive Lie group and let $P$ be a parabolic subgroup. In the terminology of Definition 1.1, suppose that $\mathcal{O}$ is a good open $G$-orbit in $X=G_{\mathbb{C}} / P_{\mathbb{C}}$ and let $\chi$ be a good character of $M_{\mathbb{C}}$. Does there exist a character $\omega$ of $P$ which is trivial on the identity component of $P$ such that $\mathcal{A}_{\mathcal{O}}(\chi) \hookrightarrow{ }^{u} \operatorname{Ind}_{P}^{G}\left(\xi_{2 \delta} \otimes \chi \omega\right)$ ?

If the nilradical of $P$ is commutative, the answer is known by [Sah92], [SS90], [Sah95], and [Zha95]. For instance, suppose that $G$ is the rank $n$ symplectic group $\operatorname{Sp}(n, \mathbb{R})$ and $P$ is the Siegel parabolic subgroup. If $n$ is even, then the cited references give an affirmative answer to the question posed in Problem D. However, if $n$ is odd, Problem D admits a negative answer, except of course for the holomorphic and antiholomorphic discrete series as mentioned above.

For quaternionic discrete series, an affirmative answer to Problem D is established in [Wal03].

The following is the affirmative answer to Problem D for an arbitrary parabolic subgroup of $\mathrm{U}(m, n)$.

Theorem E (Theorem 7.5). Let $G=\mathrm{U}(m, n)$ and let $P$ be a parabolic subgroup. Recall the terminology of Definition 1.1. Let $\chi$ be a good holomorphic character of $M_{\mathbb{C}}$ and let $\mathcal{G}$ denote the set of the good orbits in $X_{P}=G_{\mathbb{C}} / P_{\mathbb{C}}$. Then we have the following description of the socle

$$
\operatorname{Socle}\left({ }^{u} \operatorname{Ind}_{P}^{G}\left(\xi_{2 \delta_{P}} \otimes \chi\right)\right)=\bigoplus_{\mathcal{V} \in \mathcal{G}} A_{\mathcal{V}}(\chi)
$$

For $\operatorname{Sp}(p, q)$ and $\mathrm{SO}^{*}(2 n)$, the situation is similar.

Theorem F (Theorems 6.18 and 6.19). Let $G=\operatorname{Sp}(p, q)$ (respectively, $\mathrm{SO}^{*}(2 n)$ ) and let $P=M N$ be a parabolic subgroup. Suppose that $M$ has the following form

$$
M=\operatorname{GL}\left(k_{1}, \mathbb{H}\right) \times \cdots \times \operatorname{GL}\left(k_{s}, \mathbb{H}\right) \times \operatorname{Sp}\left(p^{\prime}, q^{\prime}\right),
$$

respectively,

$$
M=\mathrm{GL}\left(k_{1}, \mathbb{H}\right) \times \cdots \times \mathrm{GL}\left(k_{s}, \mathbb{H}\right) \times \mathrm{SO}^{*}\left(2 n^{\prime}\right) .
$$

For each positive integer $\ell$, let $m_{M}(\ell)$ denote the number of $i$ such that $k_{i}=\ell$. Furthermore, assume that for each $\ell>p^{\prime}+q^{\prime}$ (respectively, $\left.\ell \leqslant p^{\prime}+q^{\prime}\right), m_{M}(\ell)$ is even. Let $\chi$ be a good holomorphic character of $M_{\mathbb{C}}$. Again let $\mathcal{G}$ denote the set of the good orbits in $X_{P}=G_{\mathbb{C}} / P_{\mathbb{C}}$. Then,

$$
\operatorname{Socle}\left({ }^{u} \operatorname{Ind}_{P}^{G}\left(\xi_{2 \delta_{P}} \otimes \chi\right)\right)=\bigoplus_{\mathcal{V} \in \mathcal{G}} A_{\mathcal{V}}(\chi) .
$$

We also consider the analogous problem for $\operatorname{GL}(n, \mathbb{H})$ and for complex semisimple groups in $\S \S 8$ and 9 . 


\section{H. Matumoto and P. E. Trapa}

\section{Background and notation}

\subsection{General notation}

As usual we denote the quaternionic field, the complex number field, the real number field, the rational number field, the ring of integers, and the set of non-negative integers by $\mathbb{H}, \mathbb{C}, \mathbb{R}, \mathbb{Q}, \mathbb{Z}$, and $\mathbb{N}$, respectively. For a ring $A$ and a left $A$-module $M$, we let $\operatorname{Ann}_{A}(M)$ denote the annihilator of $M$ in $A$.

Let $H$ be a real linear Lie group and let $H_{\mathbb{C}}$ denote its complexification. We write $\mathfrak{h}_{0}$ for its Lie algebra and $\mathfrak{h}$ for its complexification. Given a subalgebra $\mathfrak{h}_{0}^{\prime}$ of $\mathfrak{h}_{0}$, we let $H^{\prime}$ denote the corresponding analytic subgroup of $H$, and adopt analogous conventions for subalgebras of $\mathfrak{h}$ and analytic subgroups of $H_{\mathbb{C}}$. We let $\mathrm{U}(\mathfrak{h})$ denote the universal enveloping algebra of $\mathfrak{h}$.

We let $G$ denote a real reductive linear Lie group with a Cartan involution $\theta$. We write $K$ for the maximal compact subgroup of $G$ corresponding to $\theta$. The corresponding complexified Cartan decomposition is denoted by $\mathfrak{g}=\mathfrak{k} \oplus \mathfrak{s}$. The subgroup $K_{\mathbb{C}}$ acts via Ad on $\mathfrak{s}$.

For a complex reductive Lie algebra $\mathfrak{g}$ and its Cartan subalgebra $\mathfrak{h}$, we let $\Delta(\mathfrak{g}, \mathfrak{h})$ denote the root system for $(\mathfrak{g}, \mathfrak{h})$. For $\alpha \in \Delta(\mathfrak{g}, \mathfrak{h})$, we let $\alpha^{\vee}$ denote the corresponding coroot in $\mathfrak{h}^{*}$.

We let $\mathcal{P}$ denote the set of integral weights,

$$
\mathcal{P}=\left\{\lambda \in \mathfrak{h}^{*} \mid\left\langle\lambda, \alpha^{\vee}\right\rangle \in \mathbb{Z} \text { for all } \alpha \in \Delta(\mathfrak{g}, \mathfrak{h})\right\} .
$$

For a connected reductive complex linear group $G_{\mathbb{C}}$ whose Lie algebra is $\mathfrak{g}$, we set $\mathcal{P}_{G_{\mathbb{C}}}=\left\{\lambda \in \mathfrak{h}^{*} \mid \lambda\right.$ appears as a weight of some finite dimensional representation of $\left.G_{\mathbb{C}} \cdot\right\}$.

\subsection{Nilpotent orbits}

Let $G$ be a linear reductive Lie group. We frequently use an invariant form to identify $\mathfrak{g}_{0}$ and $\mathfrak{g}_{0}^{*}$ (or $\mathfrak{g}$ and $\mathfrak{g}^{*}$ ) without comment. We let $\mathcal{N}_{0}$ denote the nilpotent cone in $\mathfrak{g}_{0}$, and write $\mathcal{N}$ for the nilpotent cone in $\mathfrak{g}$. There are only a finite number of $G_{\mathbb{C}}$ orbits on $\mathcal{N}$ and a finite number of $G$ orbits on $\mathcal{N}_{0}$. Let $\mathcal{N}(\mathfrak{s})=\mathcal{N} \cap \mathfrak{s}$. The action of $K_{\mathbb{C}}$ on $\mathfrak{s}$ preserves $\mathcal{N}(\mathfrak{s})$, and there are only a finite number of $K_{\mathbb{C}}$ orbits on $\mathcal{N}(\mathfrak{s})$.

Fix an orbit $\mathcal{O}$ of $G_{\mathbb{C}}$ on $\mathcal{N}$. A $K$-form of $\mathcal{O}$ is defined to be a $K_{\mathbb{C}}$ orbit on $\mathcal{O} \cap \mathfrak{s}$. We denote the set of $K$ forms of $\mathcal{O}$ by $\operatorname{Irr}(\mathcal{O} \cap \mathfrak{s})$. If $K$ is connected, this notation causes no confusion since it is indeed the case that the irreducible components of $\mathcal{O} \cap \mathfrak{s}$ are precisely the $K_{\mathbb{C}}$ orbits on $\mathcal{O} \cap \mathfrak{s}$. However, if $K$ is disconnected, a typical $K_{\mathbb{C}}$ orbit is a union of such irreducible components. According to [Vog91], each $K$ form of $\mathcal{O}$ is a Lagrangian subvariety of $\mathcal{O}$ and, hence, $\operatorname{Irr}(\mathcal{O} \cap \mathfrak{s})$ is equidimensional.

On the other hand, a real form of $\mathcal{O}$ is defined to be an orbit of $G$ on $\mathcal{O} \cap \mathfrak{g}$. We denote this set by $\operatorname{Irr}\left(\mathcal{O} \cap \mathfrak{g}_{0}\right)$ and a similar caveat applies to this notation.

The Kostant-Sekiguchi correspondence provides a bijection between the set of $K_{\mathbb{C}}$ orbits on $\mathcal{N}(\mathfrak{s})$ and the set of $G$ orbits on $\mathcal{N}_{0}$ (see, e.g., [CM94, ch. 9]). Given a $K_{\mathbb{C}}$ orbit $\mathcal{O}_{K}$, we write $\mathbf{K S}\left(\mathcal{O}_{K}\right)$ for the corresponding real orbit. In fact, for a fixed complex orbit $\mathcal{O}$, the correspondence restricts to a bijection of $\operatorname{Irr}\left(\mathcal{O} \cap \mathfrak{g}_{0}\right)$ and $\operatorname{Irr}(\mathcal{O} \cap \mathfrak{s})$, the real and $K$ forms of $\mathcal{O}$.

\subsection{Primitive ideals}

Let $\mathfrak{g}$ denote a complex reductive Lie algebra. Let $\mathfrak{h}$ denote a Cartan subalgebra in $\mathfrak{g}$ and write $W$ for the Weyl group of $\mathfrak{h}$ in $\mathfrak{g}$. Each infinitesimal character $\lambda \in \mathfrak{h}^{*} / W$ parameterizes a maximal ideal $Z_{\lambda}$ in the center $\mathrm{Z}(\mathfrak{g})$ of $\mathrm{U}(\mathfrak{g})$. 


\section{LARGE IRREDUCIBLE CONSTITUENTS OF DEGENERATE PRINCIPAL SERIES}

Recall that a two-sided ideal in $U(\mathfrak{g})$ is called primitive if it is the annihilator of a simple $U(\mathfrak{g})$ module. If we write $\operatorname{Prim}(\mathfrak{g})$ for the set of primitive ideals in $U(\mathfrak{g})$, then we have

$$
\operatorname{Prim}(\mathfrak{g})=\coprod_{\lambda \in \mathfrak{h}^{*} / W} \operatorname{Prim}_{\lambda}(\mathfrak{g}),
$$

where $\operatorname{Prim}_{\lambda}(\mathfrak{g})$ consists of the subset of primitive ideals containing $Z_{\lambda}$. If $I \in \operatorname{Prim}_{\lambda}(\mathfrak{g})$, then $I$ is said to have infinitesimal character $\lambda$. For each $\lambda$ there is a unique maximal ideal $\operatorname{Jmax}^{\max }(\lambda) \in \operatorname{Prim}_{\lambda}(\mathfrak{g})$.

Given a primitive ideal $I$ in $\mathrm{U}(\mathfrak{g})$, one may consider its associated variety $\operatorname{AV}(I) \subset \mathfrak{g}^{*} \cdot \operatorname{AV}(I)$ is defined to be the closed points of the support of the $\mathrm{S}(\mathfrak{g})=\operatorname{gr} \mathrm{U}(\mathfrak{g})$ module gr $I$ where the gradings are provided by the degree filtration on $\mathrm{U}(\mathfrak{g})$. In fact, $\mathrm{AV}(I)$ is the closure of a single nilpotent orbit in $\mathfrak{g}^{*}$ (see $[\mathrm{Jos} 85]$ ).

\subsection{Associated varieties, asymptotic supports, and the Barbasch-Vogan conjecture}

Let $\mathfrak{g}$ be a complex reductive Lie algebra, let $X$ be a finitely generated $U(\mathfrak{g})$-module, and let $\left(X^{j}\right)$ be a good filtration of $X$. The corresponding graded object $\operatorname{gr} X$ is a finitely generated $\operatorname{grU}(\mathfrak{g})$ module, hence, $\mathrm{S}(\mathfrak{g})$ module. The associated variety $\mathrm{AV}(X)$ of $X$ is defined to be the support of $\operatorname{gr} X$; see [Vog91].

Hereafter, we assume that $X$ is a Harish-Chandra $(\mathfrak{g}, K)$-module. It is not difficult to show that $\mathrm{AV}(X)$ is a union of closures of $K_{\mathbb{C}}$ orbits on $\mathcal{N}(\mathfrak{s})$, and we may write

$$
\operatorname{AV}(X)=\overline{\mathcal{O}_{K}^{1}} \cup \cdots \cup \overline{\mathcal{O}_{K}^{j}}
$$

with each $\mathcal{O}_{K}^{j}$ a $K_{\mathbb{C}}$ orbit on $\mathcal{N}(\mathfrak{s})$. By keeping track of the rank of gr $X$ along each irreducible component $\mathcal{O}_{K}^{j}$, we obtain an integral linear combination

$$
\mathcal{A V}(X)=\sum_{\mathcal{O}_{K}} n_{\mathcal{O}_{K}}\left[\mathcal{O}_{K}\right]
$$

where the sum is over $K_{\mathbb{C}}$ orbits on $\mathcal{N}(\mathfrak{s})$ and each $n$ is an integer. We call $\mathcal{A V}(X)$ the associated cycle of $X$. The associated cycle construction is additive on short exact sequences, and hence descends to the Grothendieck group of virtual Harish-Chandra modules. If $X$ is annihilated by a primitive ideal $I$ (for instance, if $X$ is irreducible), then (in the terminology of $\S 2.2$ ) each of the orbits $\mathcal{O}_{K}^{j}$ appearing in $\mathrm{AV}(X)$ is in fact a $K$ form of the complex orbit $\mathcal{O}$ which is dense in the associated variety of the annihilator of $X$ (see [Vog91]).

Nilpotent orbits also enter the representation theory of $X$ as follows. According to [BV80], the distribution character of $X$ has an asymptotic expansion whose leading term is a linear combination of Fourier transforms of canonical measures of orbits of $G$ on $\mathcal{N}_{0}$. This linear combination is called the asymptotic cycle of $X$ and is denoted by $\mathcal{A S}(X)$.

Recall the Kostant-Sekiguchi correspondence KS of $\S 2.1$. The Barbasch-Vogan conjecture (now a theorem due to Schmid and Vilonen [SV00]) asserts that

$$
\mathcal{A V}(X)=\sum n_{\mathcal{O}_{K}}\left[\mathcal{O}_{K}\right]
$$

if and only if

$$
\mathcal{A S}(X)=\sum n_{\mathcal{O}_{K}}\left[\mathbf{K S}\left(\mathcal{O}_{K}\right)\right]
$$

For a finitely generated $U(\mathfrak{g})$-module $X$, we let $\operatorname{Dim}(X)$ denote the Gelfand-Kirillov dimension of $X$ (cf. [Vog78, $\operatorname{Vog} 91])$. It is well-known that $\operatorname{Dim}(X)=\operatorname{dim} \operatorname{AV}(X)$.

For a Harish-Chandra $(\mathfrak{g}, K)$-module $V$, we let $[V]$ denote the distribution character of $V$. Let $V$ and $M$ be Harish-Chandra $(\mathfrak{g}, K)$-modules. We write $V \approx M$, if a virtual character $[V]-[M]$ 


\section{H. Matumoto and P. E. Trapa}

is a linear combination of the distribution characters of irreducible Harish-Chandra modules whose Gelfand-Kirillov dimensions are strictly smaller than $\operatorname{Dim}(V)$.

\subsection{Induced representations}

Let $G$ be a real reductive Lie group. For a parabolic subgroup $P$ of $G$, we define (unnormalized and normalized) induction as follows. Write $P=M N$ for the Levi decomposition of $P$ (and assume that $M$ is $\theta$-stable). Let $Z$ be a $(\mathfrak{l}, M \cap K)$-module and $(\pi, H)$ a Hilbert space globalization of $Z$. Define ${ }^{u} \operatorname{Ind}_{P}^{G}(Z)$ to be the $K$-finite part of

$$
\left\{f \in C^{\infty}(G) \otimes H \mid f(g \ell n)=\pi\left(\ell^{-1}\right) f(g)(g \in G, \ell \in M, n \in N)\right\} .
$$

We regard ${ }^{u} \operatorname{Ind}_{P}^{G}(Z)$ as a Harish-Chandra $(\mathfrak{g}, K)$-module as usual. In addition, we also consider the normalized induction

$$
{ }^{n} \operatorname{Ind}_{P}^{G}(Z)={ }^{u} \operatorname{Ind}_{P}^{G}\left(Z \otimes \mathbb{C}_{\delta_{P}}\right)
$$

where $\left(\delta_{P}, \mathbb{C}_{\delta_{P}}\right)$ is a one-dimensional representation of $P$ defined as follows. Let $M={ }^{o} M A$ be the Langlands decomposition of $M$ such that ${ }^{\circ} M$ and $A$ are $\theta$-stable. We let $\mathfrak{a}_{0}$ denote the Lie algebra of $A$ and $\log : A \rightarrow \mathfrak{a}_{0}$ the inverse of the exponential map. We put

$$
\delta_{P}(\operatorname{man})=e^{\frac{1}{2} \operatorname{tr}\left(\left.\operatorname{ad}(\log a)\right|_{\mathfrak{n}}\right)} \quad\left(m \in{ }^{o} M, a \in A, n \in N\right) .
$$

\subsection{Harish-Chandra cells}

For Harish-Chandra $(\mathfrak{g}, K)$-modules $X$ and $Y$, we write $X \lesssim Y$ if there exists a finite-dimensional irreducible $G$-representation $E$ such that $Y$ is isomorphic to a subquotient of $X \otimes E$. We write $X \sim Y$ if both $X \lesssim Y$ and $Y \lesssim X$ hold. It is not hard to show that $X \lesssim Y$ implies that $\operatorname{AV}(Y) \subseteq \operatorname{AV}(X)$, and so $X \sim Y$ implies that $\operatorname{AV}(Y)=\operatorname{AV}(X)$.

We fix a positive system of $\Delta(\mathfrak{g}, \mathfrak{h})$ and let $\rho$ denote the half-sum of all of the positive roots; so $\rho$ is the infinitesimal character of the trivial representation. We let $\mathcal{E}$ denote the space of invariant eigendistribution on $G$ with infinitesimal character $\rho ; \mathcal{E}$ has a basis

$$
\mathbb{B}=\{[V] \mid V \text { is an irreducible Harish-Chandra module with infinitesimal character } \rho\} .
$$

We let $W$ denote the Weyl group for $(\mathfrak{g}, \mathfrak{h}) ; W$ acts on $\mathcal{E}$ via the coherent continuation representation [Zuc77].

For each $[V] \in \mathbb{B}$, we write $\operatorname{Cone}(V)$ for the $\mathbb{C}$-subspace of $\mathcal{E}$ spanned by $\{[X] \in \mathbb{B} \mid V \lesssim X\}$. Then Cone $(V)$ is a $W$-submodule of $\mathcal{E}$. Moreover, if $V \lesssim X$, then $\operatorname{Cone}(X) \subseteq \operatorname{Cone}(V)$. Set

$$
\operatorname{Cell}(V)=\operatorname{Cone}(V) / \sum_{X \in \mathbb{B}, V \aleph_{\infty} X} \operatorname{Cone}(X) \text {. }
$$

If we write $[Y]$ again for the image of $[Y] \in \operatorname{Cone}(V)$ in $\operatorname{Cell}(V), \operatorname{Cell}(V)$ has a basis $\{[Y] \in \mathbb{B} \mid V \sim Y\}$. A Harish-Chandra cell is a subquotient of $\mathcal{E}$ which is of the form $\operatorname{Cell}(V)$ for some $[V] \in \mathbb{B}$. Let $V$ be any irreducible Harish-Chandra $(\mathfrak{g}, K)$-module whose infinitesimal character is in $\mathcal{P}_{G_{\mathbb{C}}}$. A standard application of the translation principle implies that there exists some $[X] \in \mathbb{B}$ such that $V \sim X$. We write $\operatorname{Cell}(V)$ for $\operatorname{Cell}(X)$.

\section{Associated cycles of degenerate principal series}

We begin by recalling how to compute the asymptotic cycle of a parabolically induced representation. A much more general statement is contained in [Bar00, Corollary 5.0.10]. We extract only what we need for applications. 


\section{LARGE IRREDUCIBLE CONSTITUENTS OF DEGENERATE PRINCIPAL SERIES}

Theorem 3.1 (Barbasch). Suppose that $G$ is a real reductive linear group with parabolic subgroup $P=M N$. Let $\chi$ denote a character of $M$, and consider the parabolically induced representation $\operatorname{Ind}_{P}^{G}(\chi)$.

(1) Let $\mathcal{O}_{0}$ denote a real nilpotent orbit for $\mathfrak{g}_{0}$. Then $\overline{\mathcal{O}_{0}}$ appears in the asymptotic support of $\operatorname{Ind}_{P}^{G}(\chi)$ if and only if $\mathcal{O}_{0} \cap \mathfrak{n}_{0}$ is nonempty and open in $\mathfrak{n}_{0}$.

(2) Let $P_{\mathbb{C}}$ denote the complexification of $P$ in $G_{\mathbb{C}}$. Suppose that the moment map from the cotangent bundle $T^{*}\left(G_{\mathbb{C}} / P_{\mathbb{C}}\right)$ is birational onto its image. Then if $\mathcal{O}_{0}$ appears in the asymptotic support of $\operatorname{Ind}_{P}^{G}(\chi)$, its multiplicity is exactly one.

We immediately obtain a multiplicity one result.

COROLlary 3.2. Retain the setting of Theorem 3.1(2); in particular, assume the indicated moment map is birational. If $X$ is an irreducible constituent of $\operatorname{Ind}_{P}^{G}(\chi)$ of maximal Gelfand-Kirillov dimension, then $X$ appears with multiplicity one. Moreover, if $X$ and $Y$ are irreducible constituents of $\operatorname{Ind}_{P}^{G}(\chi)$ of maximal Gelfand-Kirillov dimension such that $\operatorname{AV}(X)=\operatorname{AV}(Y)$, then we have $X=Y$.

For certain groups, the above results give a complete description of associated cycles of degenerate principal series. The main result of this section is as follows.

Proposition 3.3. Recall the notation of $\S 2.2$.

(1) Let $G$ be $\mathrm{U}(p, q), \operatorname{Sp}(p, q)$, or $\mathrm{SO}^{*}(2 n)$. Let $P$ be a parabolic subgroup of $G$ and $\chi$ be a onedimensional representation of $P$. Then

$$
\mathcal{A} \mathcal{V}\left(\operatorname{Ind}_{P}^{G}(\chi)\right)=\sum_{\mathcal{O}_{K} \in \operatorname{Irr}(\mathcal{O} \cap \mathfrak{s})}\left[\overline{\mathcal{O}_{K}}\right]
$$

and

$$
\mathcal{A S}\left(\operatorname{Ind}_{P}^{G}(\chi)\right)=\sum_{\mathcal{O}_{\mathbb{R}} \in \operatorname{Irr}\left(\mathcal{O} \cap \mathfrak{g}_{0}\right)}\left[\overline{\mathcal{O}_{\mathbb{R}}}\right] .
$$

(2) Let $G$ be $\operatorname{GL}(n, \mathbb{R})$ or $\mathrm{GL}(n, \mathbb{H})$. Let $P$ be a parabolic subgroup of $G$ and $\chi$ be a one-dimensional representation of $P$. Then $\mathcal{O} \cap \mathfrak{s}$ consists of a single $K_{\mathbb{C}}$ orbit $\mathcal{O}_{K}^{1}$ and likewise $\mathcal{O} \cap \mathfrak{g}_{0}$ consists of a simple $G$ orbit $\mathcal{O}_{\mathbb{R}}^{1}$, and

$$
\mathcal{A} \mathcal{V}\left(\operatorname{Ind}_{P}^{G}(\chi)\right)=\left[\overline{\mathcal{O}_{K}^{1}}\right]
$$

and

$$
\mathcal{A S}\left(\operatorname{Ind}_{P}^{G}(\chi)\right)=\left[\overline{\mathcal{O}_{\mathbb{R}}^{1}}\right] .
$$

Proof. The equivalence of the associated cycle and asymptotic cycle statements follows from the Barbasch-Vogan conjecture ( $\S 2.4)$. The statement in part (2) asserting that $\mathcal{O} \cap \mathfrak{s}$ and $\mathcal{O} \cap \mathfrak{g}_{0}$ are single orbits follows essentially from the existence of Jordan canonical form (see also [CM94, ch. 9]). Finally, using [Hes78], it is easy to verify that the birationality hypothesis in Theorem 3.1(2) is satisfied for each of the degenerate principal series considered in the proposition. Thus, if an orbit $\mathcal{O}_{K}$ contributes to the associated cycle in (3.1) and (3.2), it necessarily appears with multiplicity one.

Combining the above statements gives a proof of part (2) of the proposition. To finish the proof of part (1), it only remains to prove that each orbit indicated in (3.1) actually appears.

We suppose $G=\mathrm{U}(p, q)$. Let $P$ be a parabolic subgroup of $G$. Since $\mathcal{A} \mathcal{V}\left(\operatorname{Ind}_{P}^{G}(\chi)\right)$ does not depend on $\chi$, we assume that $\chi$ is a one-dimensional unitary representation of $P$ such that ${ }^{n} \operatorname{Ind}_{P}^{G}(\chi)$ has an integral infinitesimal character. Then, ${ }^{n} \operatorname{Ind}_{P}^{G}(\chi)$ is decomposed into a direct sum of derived 


\section{H. Matumoto and P. E. Trapa}

functor modules [Mat03, Theorem 3.3.1] explicitly. We may calculate the associated variety of each irreducible constituent of ${ }^{n} \operatorname{Ind}_{P}^{G}(\chi)$ via an algorithm described in [Tra01]. It is straightforward to check the desired conclusion in this case.

For $G=\operatorname{Sp}(m, n)$ or $\mathrm{SO}^{*}(2 n)$, using Theorem 3.1(1), we are reduced to the following computation.

Lemma 3.4. Let $G$ be $\operatorname{Sp}(m, n)$ or $\mathrm{SO}^{*}(2 n)$. Let $P=M N$ be a parabolic subgroup for the real group $G$. Write $\mathcal{O}$ for the Richardson orbit with respect to the complexified Lie algebra $\mathfrak{p}$ of $P$. Let $\mathcal{O}_{0}$ be a real form of $\mathcal{O}$ with respect to $G$ (see $\S 2.2$ ). Then, $\mathcal{O}_{0} \cap \mathfrak{n}_{0}$ is nonempty and open in $\mathfrak{n}_{0}$.

To prove Lemma 3.4, we first develop a general result (Lemma 3.7). So let $G$ be an arbitrary real reductive Lie group, and let $X \in \mathfrak{g}_{0}$ be a nilpotent element. Extend $X$ to a $\mathfrak{s l}_{2}$-triple $(X, H, Y)$. Let $\mathfrak{p}_{0}(X)$ denote the parabolic subalgebra consisting of the nonnegative eigenvalues of $\operatorname{ad}(H)$ on $\mathfrak{g}_{0}$. Recall that $X$ is even if the eigenvalues of $\operatorname{ad}(H)$ are all even.

Definition 3.5. A parabolic subalgebra $\mathfrak{p}_{0}$ of $\mathfrak{g}_{0}$ is called even if there exists an even nilpotent element $X$ such that $\mathfrak{p}_{0}=\mathfrak{p}_{0}(X)$. A parabolic subalgebra $\mathfrak{p}_{0}$ of $\mathfrak{g}_{0}$ is called quasieven if there exists an even parabolic subalgebra $\mathfrak{p}_{0}^{\prime}$ such that $\mathfrak{p}_{0}$ and $\mathfrak{p}_{0}^{\prime}$ have a common Levi part. Finally, a parabolic subgroup $P$ of $G$ is called quasieven if its Lie algebra is quasieven.

Example 3.6. Consider the parabolic subgroups appearing in Definition 6.6 below.

(1) The parabolic subgroup for $\operatorname{Sp}(p, q)$ is quasieven if $n_{i} \leqslant p^{\prime}+q^{\prime}$ for all $i$.

(2) The parabolic subgroup for $\mathrm{SO}^{*}(2 n)$ is quasieven if $n_{i} \leqslant n^{\prime}$ for all $i$ or $n^{\prime}=0$.

Lemma 3.7. Let $\mathfrak{p}_{0}=\mathfrak{m}_{0} \oplus \mathfrak{n}_{0}$ be any quasieven parabolic subalgebra of $\mathfrak{g}_{0}$. Let $\mathcal{O}$ denote the complex Richardson orbit induced from $\mathfrak{l}$. Then each real form of $\mathcal{O}$ intersects $\mathfrak{n}_{0}$ in a dense set.

Proof. If $\mathfrak{p}_{0}$ is an even parabolic subalgebra, then the conclusion of the lemma follows immediately from the Dynkin-Kostant theory. (Here it is important to remark that the Jacobson-Morozov theorem holds for real reductive Lie algebras and real $\mathfrak{s l}(2)$ triples.) Let $\mathfrak{p}_{0}$ be any quasieven parabolic subalgebra. Let $\mathfrak{p}_{0}^{\prime}$ be an even parabolic subalgebra such that $\mathfrak{p}_{0}$ and $\mathfrak{p}_{0}^{\prime}$ have a common Levi part. We write $\mathfrak{n}_{0}$ (respectively, $\mathfrak{n}_{0}^{\prime}$ ) for the nilradical of $\mathfrak{p}_{0}$ (respectively, $\mathfrak{p}_{0}^{\prime}$ ). Arguing as in [CM94, Theorem 7.1.3], it follows that $\operatorname{Ad}(G) \mathfrak{n}_{0}=\operatorname{Ad}(G) \mathfrak{n}_{0}^{\prime}$. The lemma follows.

Example 3.8. For $\mathfrak{g}_{0}=\mathfrak{s p}(2, \mathbb{R})$ (rank 2), there are two maximal parabolic subalgebras (up to the conjugation), namely the Siegel and Jacobi parabolics. The Siegel parabolic subalgebra is even; the Jacobi is not even quasieven. In this case, the corresponding Richardson orbit is subregular nilpotent orbit and there are three real forms. The nilradical of the Jacobi parabolic subalgebra intersects only one of them. Thus, the conclusion of the lemma fails for the Jacobi parabolic subalgebra indicating the necessity of the quasieven hypothesis in general.

Lemma 3.7 together with Example 3.6 for provides a proof of Lemma 3.4 for many, but not all, cases for $\operatorname{Sp}(p, q)$ and $\mathrm{SO}^{*}(2 n)$. For the remaining cases, we must supply an additional argument.

Suppose first that $G=\operatorname{Sp}(p, q)$. Let $P=M N$ with

$$
M=\operatorname{Sp}\left(p^{\prime}, q^{\prime}\right) \times \mathrm{GL}\left(n_{1}, \mathbb{H}\right) \times \cdots \times \mathrm{GL}\left(n_{k}, \mathbb{H}\right) .
$$

Set $n^{\prime}=p^{\prime}+q^{\prime}$ and let

$$
\begin{gathered}
n_{i}^{0}=\min \left(n_{i}, n^{\prime}\right) \\
n_{i}^{1}=n_{i}-n_{i}^{1}
\end{gathered}
$$


of $M$. Set $p^{0}=p^{\prime}+\sum_{i} n_{i}^{0}, q^{0}=q^{\prime}+\sum_{i} n_{i}^{0}$, and $p^{1}=q^{1}=\sum_{i} n_{i}^{1}$. Set $G^{j}=\operatorname{Sp}\left(p^{j}, q^{j}\right)$ and consider the parabolic subgroups $P^{j}=M^{j} N^{j}$ of $G^{j}$ with

$$
\begin{gathered}
M^{0}=\operatorname{Sp}\left(p^{\prime}, q^{\prime}\right) \times \mathrm{GL}\left(n_{1}^{0}, \mathbb{H}\right) \times \cdots \times \mathrm{GL}\left(n_{k}^{0}, \mathbb{H}\right) \\
M^{1}=\mathrm{GL}\left(n_{1}^{1}, \mathbb{H}\right) \times \cdots \times \mathrm{GL}\left(n_{k}^{1}, \mathbb{H}\right) .
\end{gathered}
$$

Let $\mathcal{O}^{j}$ denote the orbit for $\mathfrak{g}^{j}$ induced from there zero orbit of $\mathfrak{m}^{j}$. According to Example 3.6(2), $P^{0}$ is quasieven and, hence, every real form of $\mathcal{O}^{0}$ meets $\mathfrak{n}^{0}$. The key observation from the classification of real forms [CM94, ch. 9] is that the number of real forms of $\mathcal{O}$ is equal to the number of real forms of $\mathcal{O}^{0}$. So we can deduce the statement of Lemma 3.4 for $\mathcal{O}$ from the corresponding statement for $\mathcal{O}^{0}$. We offer a few more details.

Note that $P^{0} \times P^{1}$ embeds in $P$ in a natural way such that

$$
\mathfrak{n}_{0}^{0} \oplus \mathfrak{n}_{0}^{1} \subset \mathfrak{n}_{0} .
$$

As we remarked, $P^{0}$ is quasieven, so we can find representative $N_{1}, \ldots, N_{r} \in \mathfrak{n}_{0}^{0}$ for each of the $r$ real forms of $\mathcal{O}^{0}$. On the other hand, from the classification of real forms, $\mathcal{O}^{1}$ has a single real form. We may find a representative of it, say $N$, in $\mathfrak{n}_{0}^{1}$. With the decomposition of (3.3) in mind, consider the $r$ elements

$$
\left(N_{i}, N\right) \in \mathfrak{n}_{0} .
$$

From the classification of real forms, one may deduce that since the $N_{i}$ are not conjugate by $G^{1}$, none of the $r$ elements $\left(N_{i}, N\right)$ are conjugate by $G$. The emphasized remark of the previous paragraph thus implies that they are a complete set of representative of real forms of $\mathcal{O}$. Lemma 3.4 follows for $\operatorname{Sp}(p, q)$. A very similar argument works for $\mathrm{SO}^{*}(2 n)$. We omit the details.

This completes the proof of Lemma 3.4 and, hence, Proposition 3.3.

To conclude, we introduce some notions in the general setting.

Definition 3.9. Let $G$ be an arbitrary real reductive Lie group. An irreducible Harish-Chandra $(\mathfrak{g}, K)$-module $V$ is called quasi $P$-cofinite if there exists some irreducible finite-dimensional representation $F$ of $M$ such that $V$ is isomorphic to a subquotient of ${ }^{n} \operatorname{Ind}_{P}^{G}(F)$.

The following lemma is easily obtained from the fact that parabolic induction commutes with tensoring with a finite-dimensional representation (the 'Mackey isomorphism') together with the exactness of parabolic induction.

Lemma 3.10. Let $V$ be a quasi $P$-cofinite irreducible Harish-Chandra $(\mathfrak{g}, K)$-module and let $X$ be an irreducible Harish-Chandra $(\mathfrak{g}, K)$-module such that $V \lesssim X$ (with notation as in $\S 2.6$ ). Then $X$ is quasi P-cofinite.

\section{Correspondence of orbits}

Let $G$ be a real reductive linear Lie group and retain the notation of $\S 2.1$. Let $\mathfrak{q}$ be a parabolic subalgebra of $\mathfrak{g}$ and let $Q$ be the corresponding parabolic subgroup of $G_{\mathbb{C}}$. The generalized flag manifold $G_{\mathbb{C}} / Q$ is identified naturally with the set of the parabolic subalgebras of $\mathfrak{g}$ which are

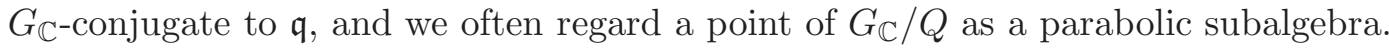

The following proposition is a special case of the Matsuki duality theorem.

Proposition 4.1 [Mat88a]. For each open $G$-orbit $\mathcal{V}$ in $G_{\mathbb{C}} / Q$, there is a unique closed $K_{\mathbb{C} \text {-orbit }}$ $\mathcal{C}$ such that $\mathcal{C} \subseteq \mathcal{V}$. This correspondence gives a bijection of the set of the open $G$-orbits in $G_{\mathbb{C}} / Q$ onto the set of the closed $K_{\mathbb{C} \text {-orbits. }}$

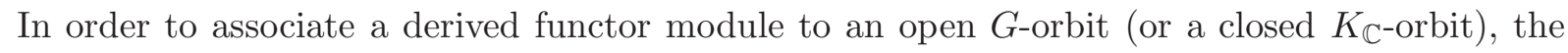
following property of the orbit is required. 


\section{H. Matumoto and P. E. Trapa}

Definition 4.2. A closed $K_{\mathbb{C}^{-o r b i t}} \mathcal{C}$ in $G_{\mathbb{C}} / Q$ is called fine if there is a $\theta$-stable parabolic subalgebra

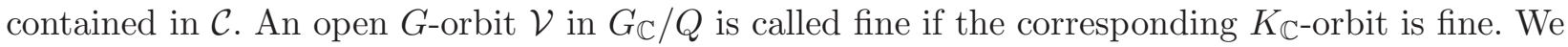
say that a parabolic subgroup $Q$ of $G_{\mathbb{C}}$ is fine with respect to $G$ if all of the open $G$-orbits in $G_{\mathbb{C}} / Q$ are fine.

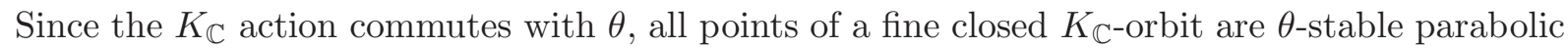
subalgebras. Also note that if $\mathfrak{q}$ is a Borel subalgebra, then all open $G$-orbits are fine [Mat79]; in other words, $Q$ is fine. Our aim here is to discuss other sufficient conditions ensuring that a particular open $G$-orbit is fine. We begin with the following easy fact.

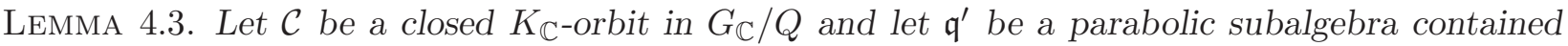
in $\mathcal{C}$. Then there exists a $\theta$-stable Borel subalgebra $\mathfrak{b}$ such that $\mathfrak{b} \subseteq \mathfrak{q}^{\prime}$.

Proof. Let $B$ be a Borel subgroup of $G_{\mathbb{C}}$ such that $B \subseteq Q$. We consider the natural projection

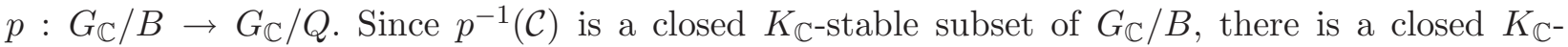

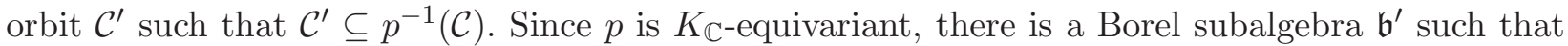
$\mathfrak{b}^{\prime} \in p^{-1}\left(\mathfrak{q}^{\prime}\right) \cap \mathcal{C}^{\prime}$. However, $\mathfrak{b}^{\prime} \in p^{-1}\left(\mathfrak{q}^{\prime}\right)$ means that $\mathfrak{b}^{\prime} \subseteq \mathfrak{q}$, and since $\mathcal{C}^{\prime}$ is fine (by the comment in the paragraph preceding the lemma), $\mathfrak{b}^{\prime}$ is $\theta$-stable. The lemma thus follows.

Proposition 4.4. Retain the notation of Definition 4.2. If $G$ has a compact Cartan subgroup, then any parabolic subgroup of $G_{\mathbb{C}}$ is fine with respect to $G$.

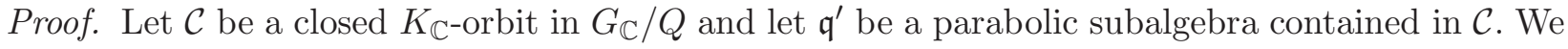
take a $\theta$-stable Borel subalgebra $\mathfrak{b}^{\prime} \subseteq \mathfrak{q}^{\prime}$ as in Lemma 4.3. Then we can choose a compact Cartan subalgebra $\mathfrak{h}$ such that $\mathfrak{h} \subseteq \mathfrak{b}^{\prime}$. Thus, $\mathfrak{q}^{\prime}$ corresponds to a subset $S$ of the set of simple roots for $\left(\mathfrak{b}^{\prime}, \mathfrak{h}\right)$. Since $\theta$ acts trivially on $\mathfrak{h}, S$ is $\theta$-stable. Hence, $\mathfrak{q}^{\prime}$ is $\theta$-stable.

We discuss another sufficient condition.

Definition 4.5. Let $\mathfrak{q}$ be a parabolic subalgebra of $\mathfrak{g}$. We choose a Borel subalgebra $\mathfrak{b}$ such that $\mathfrak{b} \subseteq \mathfrak{q}$. We call $\mathfrak{q}$ neat if $\mathfrak{q}$ is stable under any automorphism of $\mathfrak{g}$ preserving $\mathfrak{b}$. It is easy to see that the condition of being neat does not depend on the choice of $\mathfrak{b}$. A parabolic subgroup $Q$ is called neat if its Lie algebra $\mathfrak{q}$ is neat. (Lemma 4.3 implies that any neat parabolic subgroup is fine.)

We next introduce a little more notation. We let $\mu_{Q}$ denote the moment map for the cotangent

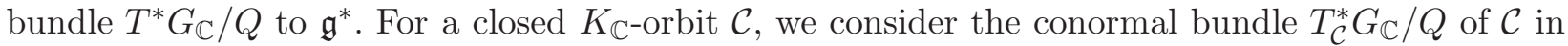
$G_{\mathbb{C}} / Q$. We let $\mathcal{C}^{\vee}$ denote the image of $T_{\mathcal{C}}^{*} G_{\mathbb{C}} / Q$ under $\mu_{Q}$. Let $\mathfrak{q}^{\prime} \in \mathcal{C}$ and write $\mathfrak{u}^{\prime}$ for the nilradical of $\mathfrak{q}^{\prime}$. After unwinding the definitions, it is easy to see that $\mathcal{C}^{\vee}=\operatorname{Ad}\left(K_{\mathbb{C}}\right)(\mathfrak{u} \cap \mathfrak{s})$; here we are implicitly identifying $\mathfrak{g}^{*}$ and $\mathfrak{g}$.

Definition 4.6. Retain the notation above. Let $\mathcal{O}$ be the Richardson orbit in $\mathfrak{g}$ with respect to $\mathfrak{q}$. A closed and fine $G$-orbit $\mathcal{C}$ is called $\operatorname{good}$ if $\operatorname{dim} \mathcal{C}^{\vee}=\operatorname{dim} \mathfrak{s} \cap \mathcal{O}$. An open $G$-orbit is called good if

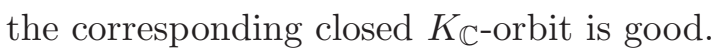

For a good closed $K_{\mathbb{C}^{-o r b i t}} \mathcal{C}, \mathcal{C}^{\vee}$ is the closure of an element of $\operatorname{Irr}(\mathcal{O} \cap \mathfrak{s})$ (with notation as in $\S 2.2)$. If the moment map $\mu_{Q}$ is birational to its image, $\mu_{Q}$ gives bijection of $\mu_{Q}^{-1}(\mathcal{O})$ onto $\mathcal{O}$, and so we have the following lemma.

Lemma 4.7. Assume that the moment map $\mu_{Q}$ is birational onto its image. Then the map $\mathcal{C} \mapsto \mathcal{C}^{\vee}$

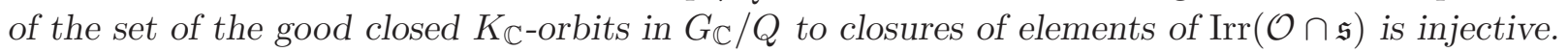

For our purposes, it is important to describe the map $\mathcal{C} \rightsquigarrow \mathcal{C}^{\vee}$. The proof of the following proposition is straightforward. 


\section{LARGE IRREDUCIBLE CONSTITUENTS OF DEGENERATE PRINCIPAL SERIES}

Proposition 4.8. Retain the notation above. Let $X$ be an even nilpotent element of $\mathfrak{g}_{0}$. Choose a $\mathfrak{s l}_{2}$-triple $(X, H, Y)$ such that $X, H, Y \in \mathfrak{g}_{0}$. Let $\mathfrak{q}$ denote the parabolic subalgebra consisting of the nonnegative eigenvalues of $\operatorname{ad}(H)$ on $\mathfrak{g}$. Define the Cayley element $C_{X} \in G_{\mathbb{C}}$ by $C_{X}=$ $\exp ((\pi i / 4)(X+Y))$. Then $\operatorname{Ad}\left(C_{X}\right) \mathfrak{q}$ is a $\theta$-stable parabolic subalgebra of $\mathfrak{g}$. Let $\mathcal{O}_{K}(X)$ denote the

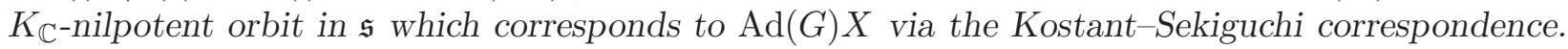

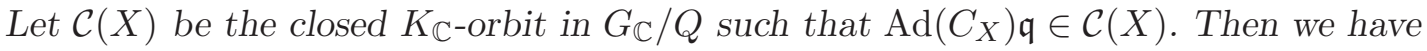

$$
\mathcal{C}(X)^{\vee}=\mathcal{O}_{K}(X) .
$$

In general, we do not know a good conceptual description of the correspondence $\mathcal{C} \rightsquigarrow \mathcal{C}^{\vee}$. However, for classical groups, some combinatorial algorithms computing Richardson orbits in real groups were obtained in [Tra01] and [Tra05] (see also [Yam97]). Examining those algorithms we obtain the following proposition.

Proposition 4.9. Let $G$ be $\mathrm{U}(m, n), \operatorname{Sp}(p, q)$, or $\mathrm{SO}^{*}(2 n)$. Consider the following Levi subalgebra $\mathfrak{l}$ of the complexified Lie algebra $\mathfrak{g}$ of $G$,

$$
\mathfrak{l} \simeq \begin{cases}\mathfrak{g l}\left(n_{1}, \mathbb{C}\right) \oplus \cdots \oplus \mathfrak{g l}\left(n_{k}, \mathbb{C}\right) & \text { if } G=\mathrm{U}(m, n), \\ \mathfrak{g l}\left(2 n_{1}, \mathbb{C}\right) \oplus \cdots \oplus \mathfrak{g l}\left(2 n_{k}, \mathbb{C}\right) \oplus \mathfrak{s p}\left(2 n^{\prime}, \mathbb{C}\right) & \text { if } G=\operatorname{Sp}(p, q), \\ \mathfrak{g l}\left(2 n_{1}, \mathbb{C}\right) \oplus \cdots \oplus \mathfrak{g l}\left(2 n_{k}, \mathbb{C}\right) \oplus \mathfrak{s o}\left(2 n^{\prime}, \mathbb{C}\right) & \text { if } G=\operatorname{SO}^{*}(2 n) .\end{cases}
$$

Let $\mathcal{O}$ denote the complex Richardson orbit induced from $\mathfrak{l}$. Let $\mathfrak{q}$ be an arbitrary parabolic subalgebra of $\mathfrak{g}$ whose Levi part is $\mathfrak{l}$ and let $Q$ denote the analytic subgroup of $G_{\mathbb{C}}$ corresponding to $\mathfrak{q}$. In this case, $Q$ is fine by Proposition 4.4.

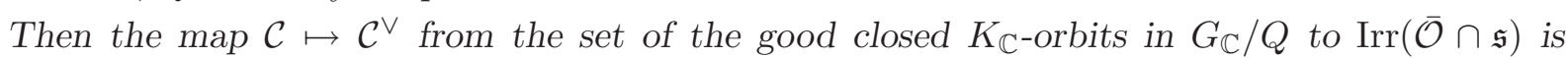
bijective. Moreover, the correspondence $\mathcal{C} \rightsquigarrow \mathcal{C}^{\vee}$ is described explicitly by the combinatorial data described in [Tra01] and [Tra05].

\section{Weakly unipotent primitive ideals associated to a Richardson orbit}

We briefly recall some features of the theory of unipotent primitive ideals. The contents of this section are more or less known (but not collected in one place). Definitions 5.1 and 5.3 provide the notion of weak unipotence and distinguished unipotence for primitive ideals. Propositions 5.5 gives a nice characterizing of distinguished unipotence for primitive ideals whose associated variety is Richardson. Again in the context of Richardson orbits, Proposition 5.7 shows that indeed the notions of weak and distinguished unipotence coincide. (In general, the latter implies the former, but not conversely.) We then turn to specific examples. The main results needed for applications below are Proposition 5.10, 5.12, and 5.14. These may be also deduced from the classification of primitive ideals for classical algebras, but we prefer to avoid invoking the classification.

Let $\mathfrak{g}$ denote a complex reductive Lie algebra and recall the notation of $\S 2.3$. Set $\mathfrak{h}^{s s}=\mathfrak{h} \cap[\mathfrak{g}, \mathfrak{g}]$ and let $G_{\mathbb{C}}^{s s}$ denote the analytic subgroup of $G_{\mathbb{C}}$ corresponding to $[\mathfrak{g}, \mathfrak{g}]$.

Definition 5.1 ([KV95, Definition 12.3] and [Vog87, Definition 12.10]). We have the following.

(1) An irreducible $U(\mathfrak{g})$-module $X$ with an infinitesimal character $\lambda$ is called weakly unipotent if:

(a) the restriction of $\lambda$ to $\mathfrak{h}^{\text {ss }}$ is in the $\mathbb{R}$-linear span of the roots;

(b) for each finite-dimensional $\mathrm{U}(\mathfrak{g})$ module $F, X \otimes_{\mathbb{C}} F$ has finite length (as a $\mathrm{U}(\mathfrak{g})$ module); and

(c) if there exists a nonzero subquotient of $X \otimes F$ annihilated by an ideal of the form $Z_{\mu}$, then $\left\langle\left.\lambda\right|_{\mathfrak{h}^{s s}},\left.\lambda\right|_{\mathfrak{h}^{s s}}\right\rangle \leqslant\left\langle\left.\mu\right|_{\mathfrak{h}^{s s}},\left.\mu\right|_{\mathfrak{h}^{s s}}\right\rangle$.

If, in addition, $\mathcal{O}$ is dense in $\operatorname{AV}(\operatorname{Ann}(X))$, then we say that $X$ is weakly unipotent attached to $\mathcal{O}$. 


\section{H. Matumoto and P. E. Trapa}

(2) A two-sided ideal of $I$ in $\mathrm{U}(\mathfrak{g})$ is called a weakly unipotent primitive ideal if it is the annihilator of a weakly unipotent irreducible $\mathrm{U}(\mathfrak{g})$-module.

If we apply results in $[\operatorname{Vog} 90, \S 7]$, it is not difficult to deduce the following result.

Corollary 5.2. If an irreducible $\mathrm{U}(\mathfrak{g})$-module $X$ with a weakly unipotent annihilator satisfies condition (b) in Definition 5.1, then $X$ is a weakly unipotent $\mathrm{U}(\mathfrak{g})$-module.

Definition 5.3 (Compare [Vog87, Definition 12.6]). Let $\lambda \in \mathfrak{h}^{*}$ be such that $\left.\lambda\right|_{\mathfrak{h}^{\text {ss }}}$ is contained in the real linear span of the roots. Let $I \in \operatorname{Prim}_{\lambda}(\mathfrak{g})$. Let $\operatorname{AV}(I)=\overline{\mathcal{O}}$ and set

$$
\operatorname{Prim}(\mathcal{O})=\left\{I^{\prime} \in \operatorname{Prim}(\mathfrak{g}) \mid \operatorname{AV}\left(I^{\prime}\right)=\overline{\mathcal{O}}\right\},
$$

and

Finally, set

$$
\operatorname{IC}(\mathcal{O})=\left\{\lambda^{\prime} \mid \operatorname{Prim}_{\lambda^{\prime}}(\mathfrak{g}) \cap \operatorname{Prim}(\mathcal{O}) \neq \emptyset\right\}
$$

$$
\operatorname{IC}_{G_{\mathbb{C}}}(\mathcal{O}, \lambda)=\left\{\lambda^{\prime} \in \operatorname{IC}(\mathcal{O}) \mid \lambda^{\prime}-\lambda \in \mathcal{P}_{G_{\mathbb{C}}}\right\} .
$$

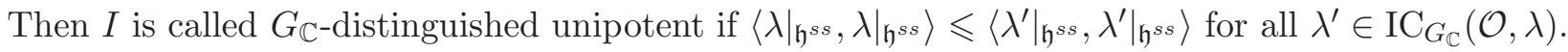
In this case, we say that $I$ is attached to $\mathcal{O}$. If the infinitesimal character of $I$ is in $\mathcal{P}_{G_{\mathbb{C}}}$, we say that $I$ is $G_{\mathbb{C}}$-integral distinguished unipotent attached to $\mathcal{O}$. We let $\operatorname{Dist}_{G_{\mathbb{C}}}(\mathcal{O})$ denote the set of the

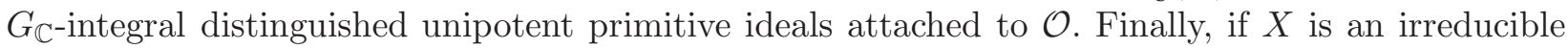
Harish-Chandra module whose annihilator is an element of $\operatorname{Dist}_{G_{\mathbb{C}}}(\mathcal{O})$, we say that $X$ is distinguished unipotent attached to $\mathcal{O}$.

We now describe a general method of constructing $G_{\mathbb{C}}$-integral distinguished unipotent primitive ideals attached to Richardson orbits. So suppose that $\mathcal{O}$ is induced from the zero orbit of a Levi factor $\mathfrak{l}$. Let $\mathfrak{h}$ be a Cartan subalgebra in $\mathfrak{l}$ (and, hence, in $\mathfrak{g}$ ) and fix a positive system $\Delta^{+}(\mathfrak{g}, \mathfrak{h})$ for $\Delta(\mathfrak{g}, \mathfrak{h})$. Let $\mathfrak{b}$ be the Borel subalgebra containing $\mathfrak{h}$ that corresponds to $\Delta^{+}(\mathfrak{g}, \mathfrak{h})$. Put $\Delta^{+}(\mathfrak{l}, \mathfrak{h})=$ $\Delta^{+}(\mathfrak{g}, \mathfrak{h}) \cap \Delta(\mathfrak{l}, \mathfrak{h})$. Write $\delta(\mathfrak{l}) \in \mathfrak{h}^{*}$ (respectively, $\rho \in \mathfrak{h}^{*}$ ) for the half-sum of positive roots of $\mathfrak{h}$ in $\mathfrak{l}$ (respectively, $\mathfrak{g}$ ). Fix a parabolic subalgebra $\mathfrak{q}$ of $\mathfrak{g}$ with Levi decomposition $\mathfrak{q}=\mathfrak{l}+\mathfrak{u}$ such that $\mathfrak{b} \subseteq \mathfrak{q}$. Set

$$
\mathcal{P}^{++}(\mathfrak{l})=\left\{\lambda \in \mathfrak{h}^{*} \mid\left\langle\lambda, \alpha^{\vee}\right\rangle \in\{1,2,3, \ldots\}\left(\alpha \in \Delta^{+}(\mathfrak{l}, \mathfrak{h})\right)\right\}
$$

and

$$
\mathcal{S}_{G_{\mathbb{C}}}(\mathfrak{l})=\left\{\lambda \in \mathcal{P}^{++}(\mathfrak{l}) \cap \mathcal{P}_{G_{\mathbb{C}}} \mid\left\langle\left.\lambda\right|_{\mathfrak{h}^{s s}},\left.\lambda\right|_{\mathfrak{h}^{s s}}\right\rangle \leqslant\left\langle\left.\mu\right|_{\mathfrak{h}^{s s}},\left.\mu\right|_{\mathfrak{h}^{s s}}\right\rangle \text { for all } \mu \in \mathcal{P}^{++}(\mathfrak{l}) \cap \mathcal{P}_{G_{\mathbb{C}}}\right\} .
$$

For $\mu \in \mathcal{P}^{++}(\mathfrak{l})$, let $E_{\mathfrak{l}}(\mu-\rho)$ denote the irreducible finite-dimensional $\mathrm{U}(\mathfrak{l})$-module with a highest weight $\mu-\rho$. Introducing the trivial action of the nilradical of $\mathfrak{q}$, we regard $E_{\mathfrak{l}}(\mu-\rho)$ as a $U(\mathfrak{q})$ module. We define the generalized Verma module induced from $E$ via

$$
M_{\mathfrak{q}}(\mu)=\mathrm{U}(\mathfrak{g}) \otimes_{\mathrm{U}(\mathfrak{q})} E_{\mathfrak{l}}(\mu-\rho) .
$$

For $\mu \in \mathfrak{h}^{*}$, write $L(\mu)$ for the irreducible $\mathrm{U}(\mathfrak{g})$-module with a highest weight $\lambda-\rho$. A $\mathrm{U}(\mathfrak{g})$-module $X$ is called $\mathfrak{q}$-finite if $\operatorname{dim} \mathrm{U}(\mathfrak{q}) v<\infty$ holds for each $v \in X$. For $\mu \in \mathcal{P}^{++}(\mathfrak{l}), L(\mu)$ is $\mathfrak{q}$-finite. Conversely, each $\mathfrak{q}$-finite irreducible $\mathrm{U}(\mathfrak{g})$-module is isomorphic to $L(\mu)$ for some $\mu \in \mathcal{P}^{++}(\mathfrak{l})$. In other words, a $\mathfrak{q}$-finite irreducible $\mathrm{U}(\mathfrak{g})$-module is a (unique) quotient of a generalized Verma module.

Lemma 5.4. Let $\mathcal{O}$ denote the Richardson orbit induced from a parabolic subalgebra $\mathfrak{q}$ of $\mathfrak{g}$. Let $I$ and $J$ be primitive ideals in $\mathrm{U}(\mathfrak{g})$ with integral infinitesimal character $\lambda$ such that $I \subseteq J$. Assume that $I \in \operatorname{Prim}(\mathcal{O})$. Then there exists some irreducible $\mathfrak{q}$-finite $\mathrm{U}(\mathfrak{g})$-module $E$ such that $J$ is the annihilator of $E$ in $\mathrm{U}(\mathfrak{g})$.

Proof. The translation principle easily reduces matters to the case of regular infinitesimal character. Since the $\mathfrak{q}$-finite irreducible $U(\mathfrak{g})$-modules with a given regular integral infinitesimal character form 


\section{LARGE IRREDUCIBLE CONSTITUENTS OF DEGENERATE PRINCIPAL SERIES}

a single right cone in category $\mathbf{O}$, the lemma follows from [LX88, Theorem 3.2]. (Actually, Lusztig$\mathrm{Xi}$ 's result is stated in the context of affine Weyl groups but the same proof is applicable to the case of finite Weyl groups. We also remark that we may of course interchange ' $L$ ' and ' $R$ ' in the statement of their result.)

Lemma 5.4 gives the following two results.

Proposition 5.5. Fix a Richardson orbit $\mathcal{O}$ induced from $\mathfrak{l}$ and retain the above notation (especially that of Definition 5.3 and (5.1)). Then

$$
\operatorname{Dist}_{G_{\mathbb{C}}}(\mathcal{O})=\operatorname{Prim}(\mathcal{O}) \cap \bigcup_{\lambda \in \mathcal{S}_{G_{\mathbb{C}}}(\mathfrak{l})} \operatorname{Prim}_{\lambda}(\mathfrak{g}) .
$$

Proposition 5.6. Let $\lambda \in \mathcal{P}^{++}(\mathfrak{l})$ be such that $\mathcal{P}^{++}(\mathfrak{l}) \cap W \lambda=\{\lambda\}$. Then:

(1) $M_{\mathfrak{q}}(\lambda)$ is irreducible and $J_{\max }(\lambda)=\operatorname{Ann}_{\mathrm{U}(\mathfrak{g})}\left(M_{\mathfrak{q}}(\lambda)\right)$;

(2) $\operatorname{Prim}_{\lambda}(\mathfrak{g}) \cap \operatorname{Prim}(\mathcal{O})=\left\{J_{\max }(\lambda)\right\}$.

Next we consider the weakly unipotent primitive ideals attached to a Richardson orbit.

Proposition 5.7. Retain the above notation. In particular, assume that $\mathcal{O}$ is induced from the zero orbit of a Levi factor $\mathfrak{l}$. Further, assume that $G_{\mathbb{C}}^{s s}$ is simply connected. Fix $\lambda \in \mathcal{S}_{G_{\mathbb{C}}}(\mathfrak{l})$ (with notation as in (5.1)). Fix $I \in \operatorname{Prim}_{\lambda}(\mathfrak{g})$ and assume that there exists some $I^{\prime} \in \operatorname{Prim}(\mathcal{O})$ such that $I^{\prime} \subseteq I$. Then $I$ is weakly unipotent. In particular, each element of $\operatorname{Dist}_{G_{\mathbb{C}}}(\mathcal{O})$ is weakly unipotent. Conversely, if $I \in \operatorname{Prim}(\mathcal{O})$ is weakly unipotent, then $I \in \operatorname{Dist}_{G_{\mathbb{C}}}(\mathcal{O})$. Consequently the notions of weakly unipotent and distinguished unipotent coincide for $\operatorname{Prim}(\mathcal{O})$.

Proof. Fix $I \in \operatorname{Prim}_{\lambda}(\mathfrak{g})$ and assume that there exists some $I^{\prime} \in \operatorname{Prim}(\mathcal{O})$ such that $I^{\prime} \subseteq I$. Then it follows from Lemma 5.4 that $I$ is the annihilator of an irreducible $\mathfrak{q}$-finite $\mathrm{U}(\mathfrak{g})$-module, say $X$. For any finite-dimensional $\mathrm{U}(\mathfrak{g})$-module $F, X \otimes_{\mathbb{C}} F$ is $\mathfrak{q}$-finite. Thus, the infinitesimal character of any irreducible subquotient of $X \otimes_{\mathbb{C}} F$ belongs $\mathcal{P}^{++}(\mathfrak{l})$ (up to the action of the Weyl group). Since $X$ is isomorphic to $L(\mu)$ for some $\mu \in \mathcal{S}_{G_{\mathbb{C}}}(\mathfrak{l})$, it follows from Definition 5.1 and the definition of (5.1) that $I$ is weakly unipotent.

Consider the converse. Let $I \in \operatorname{Prim}(\mathcal{O})$ be weakly unipotent. If $I \notin \operatorname{Dist}_{G_{\mathbb{C}}}(\mathcal{O})$, then there exists some $\mu \in \mathcal{P}^{++}(\mathfrak{h})$ such that $\mu \notin \mathcal{S}_{G_{\mathbb{C}}}(\mathfrak{l})$ and so that $I$ is the annihilator of $L(\mu)$ (by Lemma 5.4). Since the $\mathfrak{q}$-finite irreducible $\mathrm{U}(\mathfrak{g})$-modules with a given regular integral infinitesimal character form a single right cone for category $\mathbf{O}$, it follows that an irreducible generalized Verma module with respect to $\mathfrak{q}$ (say $V$ ) is an irreducible subquotient of $L(\mu) \otimes_{\mathbb{C}} F_{1}$ for some finite-dimensional $\mathrm{U}(\mathfrak{g})$ module $F_{1}$. Thus, any irreducible $\mathfrak{q}$-finite $\mathrm{U}(\mathfrak{g})$-module with an integral infinitesimal character is an irreducible constituent of $V \otimes_{\mathbb{C}} F_{2}$ for some finite-dimensional $\mathrm{U}(\mathfrak{g})$-module $F_{2}$. Since $G_{\mathbb{C}}^{s s}$ is assumed to be simply connected, $\mathcal{S}_{G_{\mathbb{C}}}(\mathfrak{l})$ is nonempty. So for $\eta \in \mathcal{S}_{G_{\mathbb{C}}}(\mathfrak{l})$, there is some finite-dimensional $\mathrm{U}(\mathfrak{g})$-module $F_{3}$ such that $L(\eta)$ is an irreducible constituent of $L(\mu) \otimes_{\mathbb{C}} F_{3}$. This contradicts the assumption that $I$ is weakly unipotent.

We now consider examples. Suppose first that $\mathfrak{g}=\mathfrak{g l}(n, \mathbb{C}), G_{\mathbb{C}}=\operatorname{GL}(n, \mathbb{C})$, and $\mathfrak{l} \cong \mathfrak{g l}\left(n_{1}, \mathbb{C}\right) \oplus$ $\cdots \oplus \mathfrak{g l}\left(n_{k}, \mathbb{C}\right)$; in coordinates

$$
\delta(\mathfrak{l})=(\overbrace{\left(n_{1}-1\right) / 2, \ldots,-\left(n_{1}-1\right) / 2}^{n_{1}}, \ldots, \overbrace{\left(n_{k}-1\right) / 2, \ldots,-\left(n_{k}-1\right) / 2}^{n_{k}}) .
$$




\section{H. Matumoto and P. E. Trapa}

Write $\mathcal{O}$ for the Richardson orbit induced from the zero orbit of $\mathfrak{l}$. We put

$$
\begin{gathered}
\varepsilon_{i}= \begin{cases}0 & \text { if } n_{i} \text { is odd, } \\
1 & \text { if } n_{i} \text { is even. }\end{cases} \\
\varepsilon=(\overbrace{\varepsilon_{1}, \ldots, \varepsilon_{1}}^{n_{1}}, \ldots, \overbrace{\varepsilon_{k}, \ldots, \varepsilon_{k}}^{n_{k}}) .
\end{gathered}
$$

The next two lemmas are simple computational exercises.

Lemma 5.8. In the above setting, $\mathcal{P}^{++}(\mathfrak{l}) \cap W \lambda=\{\lambda\}$ for all $\lambda \in \mathcal{P}^{++}(\mathfrak{l})$ such that $\left.\lambda\right|_{\mathfrak{h}^{s s}}=$ $\left.\left(\delta(\mathfrak{l}) \pm \frac{1}{2} \varepsilon\right)\right|_{\mathfrak{h}^{s s}}$.

Lemma 5.9. Retain the above setting. Put

$$
s(\mathfrak{l})=\left\{\delta(\mathfrak{l})+\eta \frac{1}{2} \varepsilon \mid \eta= \pm 1 \text { and the length of }\left.\left(\delta(\mathfrak{l})+\eta \frac{1}{2} \varepsilon\right)\right|_{\mathfrak{h}^{s s}}\right.
$$

is less than or equal to the length of $\left.\left.\left(\delta(\mathfrak{l})-\eta \frac{1}{2} \varepsilon\right)\right|_{\mathfrak{h}^{s s}}\right\}$;

i.e., roughly speaking, $s(\mathfrak{l})$ consists of the shorter of the two elements $\delta(\mathfrak{l}) \pm \frac{1}{2} \varepsilon$ (or both if they have the same length). Then

$$
\mathcal{S}_{G_{\mathbb{C}}}(\mathfrak{l})=\left\{\lambda \in \mathcal{P}^{++}(\mathfrak{l})|\lambda|_{\mathfrak{h}^{s s}}=\left.\mu\right|_{\mathfrak{h}^{s s}} \text { for some } \mu \in s(\mathfrak{l})\right\} .
$$

In particular, at least one of $\delta(\mathfrak{l}) \pm \frac{1}{2} \varepsilon$ is in $\mathcal{S}_{G_{\mathbb{C}}}(\mathfrak{l})$.

Hence, from Propositions 5.6 and 5.7, we obtain the following.

Proposition 5.10. For $G_{\mathbb{C}}=\operatorname{GL}(n, \mathbb{C})$,

$$
\operatorname{Dist}_{G_{\mathbb{C}}}(\mathcal{O})=\left\{J_{\max }(\lambda)|\lambda|_{\mathfrak{h}^{s s}}=\left.\mu\right|_{\mathfrak{h}^{s s}} \text { for some } \mu \in s(\mathfrak{l})\right\}
$$

where $s(\mathfrak{l})$ is defined in Lemma 5.8.

We now turn our attention to the cases of $G_{\mathbb{C}}=\operatorname{Sp}(n, \mathbb{C})$ or $G_{\mathbb{C}}=\operatorname{SO}(2 n, \mathbb{C})$. Fix a Cartan subgroup $\mathfrak{h}$ of $\mathfrak{g}$. Then we can choose an orthonormal basis $e_{1}, \ldots, e_{n}$ of $\mathfrak{h}^{*}$ such that

$$
\Delta(\mathfrak{g}, \mathfrak{h})= \begin{cases}\left\{ \pm e_{i} \pm e_{j} \mid 1 \leqslant i<j \leqslant n\right\} & \text { if } \mathfrak{g}=\mathfrak{s o}(2 n, \mathbb{C}) \\ \left\{ \pm e_{i} \pm e_{j} \mid 1 \leqslant i<j \leqslant n\right\} \cup\left\{ \pm 2 e_{i} \mid 1 \leqslant i \leqslant n\right\} & \text { if } \mathfrak{g}=\mathfrak{s p}(n, \mathbb{C}) .\end{cases}
$$

Let $E_{1}, \ldots, E_{n}$ be the dual basis of $\mathfrak{h}$ to $e_{1}, \ldots, e_{n}$. We fix a simple system for $\Delta(\mathfrak{g}, \mathfrak{h})$ in each case as follows. If $G_{\mathbb{C}}=\operatorname{SO}(2 n, \mathbb{C})$, then let $\Pi=\left\{e_{1}-e_{2}, \ldots, e_{n-1}-e_{n}, e_{n-1}+e_{n}\right\}$, and if $G_{\mathbb{C}}=\operatorname{Sp}(n, \mathbb{C})$, set $\Pi=\left\{e_{1}-e_{2}, \ldots, e_{n-1}-e_{n}, 2 e_{n}\right\}$. We let $\Delta^{+}$denote the corresponding positive system of $\Delta(\mathfrak{g}, \mathfrak{h})$. Let $\mathfrak{b}$ denote the corresponding Borel subalgebra containing $\mathfrak{h}$.

Fix a Levi subalgebra of the following form:

$$
\mathfrak{l} \cong \begin{cases}\mathfrak{g l}\left(2 k_{1}, \mathbb{C}\right) \oplus \cdots \oplus \mathfrak{g l}\left(2 k_{s}, \mathbb{C}\right) \oplus \mathfrak{s p}\left(n^{\prime}, \mathbb{C}\right) & \text { if } \mathfrak{g}=\mathfrak{s p}(n, \mathbb{C}) \\ \mathfrak{g l}\left(2 k_{1}, \mathbb{C}\right) \oplus \cdots \oplus \mathfrak{g l}\left(2 k_{s}, \mathbb{C}\right) \oplus \mathfrak{s o}\left(2 n^{\prime}, \mathbb{C}\right) & \text { if } \mathfrak{g}=\mathfrak{s o}(2 n, \mathbb{C})\end{cases}
$$

Set $k_{i}^{*}=k_{1}+\cdots+k_{i}$ for $1 \leqslant i \leqslant s$ and $k_{0}^{*}=0$. We assume that $\mathfrak{h} \subseteq \mathfrak{l}$ and that $E_{2 k_{i-1}^{*}+1}, E_{2 k_{i-1}^{*}+2}, \ldots, E_{2 k_{i}^{*}}$ are contained in the $\mathfrak{g l}\left(2 k_{i}, \mathbb{C}\right)$-factor in the above direct sum decomposition.

If $\mathfrak{g}=\mathfrak{s p}(n, \mathbb{C})$, in coordinates

$$
\delta(\mathfrak{l})=(\overbrace{k_{1}-\frac{1}{2}, k_{1}-\frac{3}{2}, \ldots,-k_{1}+\frac{1}{2}}^{2 k_{1}}, \ldots, \overbrace{k_{s}-\frac{1}{2}, k_{s}-\frac{3}{2}, \ldots,-k_{s}+\frac{1}{2}}^{2 k_{s}}, n^{\prime}, n^{\prime}-1, \ldots, 1) .
$$




\section{LARGE IRREDUCIBLE CONSTITUENTS OF DEGENERATE PRINCIPAL SERIES}

If $\mathfrak{g}=\mathfrak{s o}(2 n, \mathbb{C})$, in coordinates

$$
\delta(\mathfrak{l})=(\overbrace{k_{1}-\frac{1}{2}, k_{1}-\frac{3}{2}, \ldots,-k_{1}+\frac{1}{2}}^{2 k_{1}}, \ldots, \overbrace{k_{s}-\frac{1}{2}, k_{s}-\frac{3}{2}, \ldots,-k_{s}+\frac{1}{2}}^{2 k_{s}}, n^{\prime}-1, n^{\prime}-2, \ldots, 0) .
$$

Finally, let $\mathfrak{q}$ be a parabolic subalgebra with a Levi part $\mathfrak{l}$.

For $\vec{\eta}=\left(\eta_{1}, \ldots, \eta_{s}\right) \in\{ \pm 1\}^{s}$, define

$$
[\vec{\eta}]=(\overbrace{\eta_{1}, \ldots, \eta_{1}}^{2 k_{1}}, \ldots, \overbrace{\eta_{k}, \ldots, \eta_{k}}^{2 k_{s}}, \overbrace{0, \ldots, 0}^{n^{\prime}}) .
$$

Let $\mathcal{O}$ denote the Richardson orbit induced from the zero orbit of $\mathfrak{l}$. The next lemma follows easily.

LEMma 5.11. Retain the above setting. Then

$$
\mathcal{S}_{G_{\mathbb{C}}}(\mathfrak{l})=\left\{\delta(\mathfrak{l})+\frac{1}{2}[\vec{\eta}] \mid \vec{\eta} \in\{ \pm 1\}^{s}\right\} .
$$

In particular, any two elements in $\mathcal{S}_{G_{\mathbb{C}}}(\mathfrak{l})$ are conjugate under the Weyl group action.

Proposition 5.12. Retain the above setting. Set $\vec{\eta}_{0}=(\overbrace{-1, \ldots,-1}^{s}$ and $\lambda_{\mathfrak{q}}=\delta+\left[\vec{\eta}_{0}\right]$. Then

$$
\operatorname{Dist}_{G_{\mathbb{C}}}(\mathcal{O})=\left\{\operatorname{Ann}_{\mathrm{U}(\mathfrak{g})}\left(M_{\mathfrak{q}}\left(\lambda_{\mathfrak{q}}\right)\right)\right\} .
$$

Proof. From [Vog84, Proposition 8.5], $M_{\mathfrak{q}}\left(\lambda_{\mathfrak{q}}\right)$ is irreducible. Hence, from Lemma 5.11, we have $\operatorname{Ann}_{\mathrm{U}(\mathfrak{g})}\left(M_{\mathfrak{q}}\left(\lambda_{\mathfrak{q}}\right)\right) \in \operatorname{Dist}_{G_{\mathbb{C}}}(\mathcal{O})$. Let $I \in \operatorname{Dist}_{G_{\mathbb{C}}}(\mathcal{O})$. According to Proposition 5.5 and Lemma 5.11, there is some $\vec{\eta} \in\{ \pm 1\}^{s}$ such that $I=\operatorname{Ann}_{\mathrm{U}(\mathfrak{g})}(L(\delta(\mathfrak{l})+[\vec{\eta}]))$. Since $E_{\mathfrak{l}}(\delta(\mathfrak{l})+[\vec{\eta}])$ is one-dimensional, the Bernstein degree (see, e.g., $[\operatorname{Vog} 78])$ of $M_{\mathfrak{q}}(\delta(\mathfrak{l})+[\vec{\eta}])$ is one. On the other hand, since $\mathcal{O}$ is dense in the associated variety of both $L_{\mathfrak{q}}(\delta(\mathfrak{l})+[\vec{\eta}])$ and $M_{\mathfrak{q}}(\delta(\mathfrak{l})+[\vec{\eta}])$, it follows that $L_{\mathfrak{q}}(\delta(\mathfrak{l})+[\vec{\eta}])$ is the unique constituent of $M_{\mathfrak{q}}(\delta(\mathfrak{l})+[\vec{\eta}])$ of maximal Gelfand-Kirillov dimension. On the other hand, from [BJ77, 4.10 Corollar], for each $\vec{\eta} \in\{ \pm 1\}^{s}$, we have $\operatorname{Ann}_{\mathrm{U}(\mathfrak{g})}\left(M_{\mathfrak{q}}(\delta(\mathfrak{l})+[\vec{\eta}])\right)=\operatorname{Ann}_{\mathrm{U}(\mathfrak{g})}\left(M_{\mathfrak{q}}\left(\lambda_{\mathfrak{q}}\right)\right)$. Hence, we have $\operatorname{Ann}_{\mathrm{U}(\mathfrak{g})}\left(M_{\mathfrak{q}}\left(\lambda_{\mathfrak{q}}\right)\right) \subset I$, both of which have the same associated variety (the closure of $\mathcal{O})$. Then [BK76, Korollar 3.6] implies that indeed $I=\operatorname{Ann}_{\mathrm{U}(\mathfrak{g})}\left(M_{\mathfrak{q}}\left(\lambda_{\mathfrak{q}}\right)\right)$.

Definition 5.13. In the setting of Proposition 5.12, we let $I_{\mathcal{O}}$ denote the unique element of $\operatorname{Dist}_{G_{\mathbb{C}}}(\mathcal{O})$. In addition, we let $\lambda(\mathcal{O})$ denote the infinitesimal character of $I_{\mathcal{O}}$ (namely $\lambda_{\mathfrak{q}}$ ).

Proposition 5.14. Retain the above setting; in particular, recall the notation of Definition 5.1, Proposition 5.12, and Definition 5.13.

(1) If $\mathfrak{g}=\mathfrak{s p}(n, \mathbb{C})$, then $I_{\mathcal{O}}$ is the unique integral weakly unipotent primitive ideal associated to $\mathcal{O}$.

(2) If $\mathfrak{g}=\mathfrak{s o}(2 n, \mathbb{C})$ and $k_{1}+\cdots+k_{s}<n$, then $I_{\mathcal{O}}$ is the unique integral weakly unipotent primitive ideal associated to $\mathcal{O}$.

(3) If $\mathfrak{g}=\mathfrak{s o}(2 n, \mathbb{C})$ and $k_{1}+\cdots+k_{s}=n$, then $\operatorname{Ann}_{\mathrm{U}(\mathfrak{g})}\left(M_{\mathfrak{q}}(\delta(\mathfrak{l}))\right)$ is the unique integral weakly unipotent primitive ideal associated to $\mathcal{O}$.

Proof. Part (1) follows from Propositions 5.12 and 5.7. Parts (2) and (3) are obtained by applying Proposition 5.7 to $G_{\mathbb{C}}=\operatorname{Spin}(2 n, \mathbb{C})$. We omit the details.

Example 5.15. It is interesting to note that, in general, $I_{\mathcal{O}}$ (of Definition 5.13) is not necessarily maximal. For example, from [Mat03, Theorem 3.2.2 and Lemma 3.6.1], we have the following example. Let $\mathfrak{g}=\mathfrak{s p}(n, \mathbb{C})$ and $\mathfrak{l}=\mathfrak{g l}(2 k, \mathbb{C}) \oplus \mathfrak{s p}(n-2 k, \mathbb{C})$. Then $I_{\mathcal{O}}$ is maximal if and only if $3 k>n$.

Remark 5.16. Recall that if $\mathfrak{g}=\mathfrak{s o}(2 n, \mathbb{C})$ and $k_{1}+\cdots+k_{s}=n$, then the corresponding Richardson orbit is called very even. 


\section{H. Matumoto and P. E. Trapa}

\section{The groups $\operatorname{Sp}(p, q)$ and $\mathrm{SO}^{*}(2 n)$}

In this section, we assume that $G$ is either $\operatorname{Sp}(p, q)$ or $\mathrm{SO}^{*}(2 n)$ and prove the theorems outlined in the introduction relating cohomologically induced representations to certain degenerate principal series. We begin by considering some weakly unipotent derived functor modules. As a starting point, the following result follows immediately from Proposition 4.9.

Corollary 6.1. Let $G=\operatorname{Sp}(p, q)$ or $\mathrm{SO}^{*}(2 n)$. Suppose that $\mathcal{O}$ is a complex orbit induced from a Levi factor of the form appearing in Proposition 4.9. Recall the notation of $\S 2.2$ and write

$$
\operatorname{Irr}(\mathcal{O} \cap \mathfrak{s})=\left\{\mathcal{O}_{K}^{1}, \ldots, \mathcal{O}_{K}^{r}\right\} .
$$

Let $\mathfrak{p}$ be an arbitrary parabolic subalgebra of $\mathfrak{g}$ whose Levi part is $\mathfrak{l}$. Then, for each $j$ there exists a $\theta$-stable parabolic subalgebra

$$
\mathfrak{q}^{j}=\mathfrak{l}^{j} \oplus \mathfrak{u}^{j}
$$

such that $\mathfrak{p}$ and $\mathfrak{q}^{j}$ are $\operatorname{Ad}\left(G_{\mathbb{C}}\right)$-conjugate and

$$
K_{\mathbb{C}} \cdot\left(\mathfrak{u}^{j} \cap \mathfrak{s}\right)=\overline{\mathcal{O}_{K}^{j}} .
$$

Moreover,

$$
G_{\mathbb{C}} \cdot\left(\mathfrak{u}^{j} \cap \mathfrak{s}\right)=\overline{\mathcal{O}}
$$

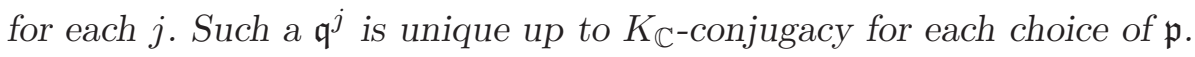

We now attach a derived functor module to each $\mathcal{O}_{K}^{j}$. Retain the setting as above and fix $\mathcal{O}_{K}^{j} \in \operatorname{Irr}(\mathcal{O} \cap \mathfrak{s})$. We fix a parabolic subalgebra $\mathfrak{p}$ of $\mathfrak{g}$ whose Levi part is $\mathfrak{l}$ as in Corollary 6.1. Let $\mathfrak{q}^{j}=\mathfrak{l}^{j} \oplus \mathfrak{u}^{j}$ be the $\theta$-stable parabolic defined by Corollary 6.1. Fix a Cartan $\mathfrak{h}$ in $\mathfrak{l}$, and choose a system of positive roots for $\mathfrak{h}$ in $\mathfrak{g}$ that contains the roots of $\mathfrak{p}$. Since $\mathfrak{p}$ and $\mathfrak{q}^{j}$ are $\operatorname{Ad}\left(G_{\mathbb{C}}\right)$-conjugate, we fix $g^{j} \in G_{\mathbb{C}}$ such that $\operatorname{Ad}\left(g^{j}\right) \mathfrak{p}=\mathfrak{q}^{j}$. Put $\mathfrak{h}^{j}=\operatorname{Ad}\left(g^{j}\right) \mathfrak{h}$. By abuse of notation, we identify $\left(\mathfrak{h}^{j}\right)^{*}$ and $\mathfrak{h}$ via $\operatorname{Ad}\left(g^{j}\right)$. Write $\delta$ for the half-sum of the positive roots. Recall the infinitesimal character $\lambda(\mathcal{O}) \in \mathfrak{h}^{*} / W$ of Definition 5.13, and choose a representative, also denoted $\lambda(\mathcal{O})$, dominant with respect to our choice of positive roots. Set

$$
\lambda^{j}=\lambda(\mathcal{O})-\delta
$$

Since $\lambda(\mathcal{O})$ differs from $\delta(\mathfrak{l})$ by a shift orthogonal to $\delta\left(\mathfrak{l}^{j}\right)$, it follow that $\lambda^{j}$ differs from $\delta\left(\mathfrak{u}^{j}\right)$ by a shift orthogonal to $\delta(\mathfrak{l})$. Moreover, $\lambda^{j}$ is integral, and hence it exponentiates to a one-dimensional $\left(\mathfrak{l}^{j}, L^{j} \cap K\right)$ module. We may thus form the derived functor module $A\left(\mathcal{O}_{K}^{j}\right):=A_{\mathfrak{q}^{j}}\left(\lambda^{j}\right)$ as in $[\mathrm{KV} 95$, ch. 5].

Proposition 6.2. Retain the setting of the previous paragraph and Corollary 6.1. Then $A\left(\mathcal{O}_{K}^{j}\right)$ is an irreducible unitary representation of $G$ with infinitesimal character $\lambda(\mathcal{O})$ (Definition 5.13). Moreover,

$$
\operatorname{AV}\left(A\left(\mathcal{O}_{K}^{j}\right)\right)=\overline{\mathcal{O}_{K}^{j}}
$$

and

$$
\operatorname{Ann}_{\mathrm{U}(\mathfrak{g})}\left(A\left(\mathcal{O}_{K}^{j}\right)\right)=I_{\mathcal{O}} .
$$

In fact, $\mathcal{O}_{K}^{j}$ occurs with multiplicity one in the associated cycle of $A\left(\mathcal{O}_{K}^{j}\right)$.

Proof. All of the assertions except the last two are contained in $[\operatorname{Vog} 88, \S 6]$. (The key hypotheses are that the induction is in the weakly fair range, see Remark 6.3, and the conclusion of (6.2).) The final assertion follows from the argument of [PT02, Proposition 6.2]. We obtain $\operatorname{Ann}_{\mathrm{U}(\mathfrak{g})}\left(A\left(\mathcal{O}_{K}^{j}\right)\right)=I_{\mathcal{O}}$ from the final assertion and Proposition 5.12 (also see [Vog86, Proposition 16.8]). 


\section{LARGE IRREDUCIBLE CONSTITUENTS OF DEGENERATE PRINCIPAL SERIES}

Remark 6.3. In the terminology of [KV95, Definition 0.52], $\lambda^{j}$ is in the weakly fair range for $\mathfrak{q}^{j}$. In fact, it is clear that $A\left(\mathcal{O}_{K}^{j}\right)$ is the most singular module possible, given that $\delta\left(\mathfrak{u}^{j}\right)$ does not exponentiate to $L^{j}$. Loosely, one may say that $A\left(\mathcal{O}_{K}^{j}\right)$ is on the edge of the weakly fair range.

Remark 6.4. The definition of $A\left(\mathcal{O}_{K}^{j}\right)$ appears to depend on the choice of $\mathfrak{p}$. Later we will see that $A\left(\mathcal{O}_{K}^{j}\right)$ indeed only depends on $\mathcal{O}_{K}^{j}$.

Next we introduce the degenerate principal series of $\operatorname{Sp}(p, q)$ and $\mathrm{SO}^{*}(2 n)$ of particular interest to us. The key is to arrange for them to have the infinitesimal character $\lambda(\mathcal{O})$ of Definition 5.13. The following lemma is a simple exercise.

Lemma 6.5. Retain the setting of Corollary 6.1. There is a Levi subgroup $M$ of $G$ such that $\mathcal{O}$ is induced from the zero orbit of the complexified Lie algebra $\mathfrak{m}$ of $M$. Such a subgroup $M$ is unique up to $\operatorname{Ad}(G)$-conjugation. Moreover, we may assume that $M$ is $\theta$-stable.

If $G=\operatorname{Sp}(p, q)$, then we have

$$
M \simeq \mathrm{GL}\left(k_{1}, \mathbb{H}\right) \times \cdots \times \mathrm{GL}\left(k_{s}, \mathbb{H}\right) \times \mathrm{Sp}\left(p^{\prime}, q^{\prime}\right)
$$

with $p=p^{\prime}+\sum k_{i}$ and $q=q^{\prime}+\sum k_{i}$.

If $G=\mathrm{SO}^{*}(2 n)$, then we have

$$
M \simeq \mathrm{GL}\left(k_{1}, \mathbb{H}\right) \times \cdots \times \mathrm{GL}\left(k_{s}, \mathbb{H}\right) \times \mathrm{SO}^{*}\left(2 n^{\prime}\right)
$$

with $n=n^{\prime}+\sum k_{i}$.

To each Richardson orbit appearing here, we define a corresponding degenerate principal series $\mathcal{I}_{P}(\mathcal{O} ; \vec{\eta})$ for each polarization and some additional data $\vec{\eta}$.

Definition 6.6. Retain the setting and notation of Lemma 6.5 and, in particular, fix a $\theta$-stable Levi subgroup $M$ as in the lemma. Let $P$ be any parabolic subgroup of $G$ with a Levi decomposition $P=M N$. For $\mathrm{GL}(n, \mathbb{H})$, we let $\operatorname{det}^{1 / 2}$ denote the following character. Given $A \in \operatorname{GL}(n, \mathbb{H})$, let $A_{\mathbb{C}}$ denote its image in $\mathrm{GL}(2 n, \mathbb{C})$ (under any of the natural embeddings). Then $\operatorname{det}\left(A_{\mathbb{C}}\right)$ is a well-defined real number, and we let $\operatorname{det}^{1 / 2}(A)$ denote its positive real fourth root. The result is a character $\operatorname{det}^{1 / 2}$ of $\mathrm{GL}(n, \mathbb{H})$ with weight $\left(\frac{1}{2}, \ldots, \frac{1}{2}\right)$. Fix $\vec{\eta}=\left(\eta_{1}, \ldots, \eta_{s}\right) \in\{ \pm 1\}^{s}$. For $G=\operatorname{Sp}(p, q)$ (respectively, $G=\mathrm{SO}^{*}(2 n)$ ), we let $\operatorname{det}^{\tilde{\eta} / 2}$ denote the character of $M$ which is trivial on $\operatorname{Sp}\left(p^{\prime}, q^{\prime}\right)$ (respectively, $\left.\mathrm{SO}^{*}\left(2 n^{\prime}\right)\right)$ but which restricts to $\operatorname{det}^{\eta_{i} / 2}$ on each $\mathrm{GL}\left(k_{i}, \mathbb{H}\right)$ factor. Finally we set

$$
\mathcal{I}_{P}(\mathcal{O} ; \vec{\eta})={ }^{n} \operatorname{Ind}_{P}^{G}\left(\operatorname{det}^{\tilde{\eta} / 2}\right),
$$

where the induction is normalized (see $\S 2.5)$.

The following lemma is immediate.

Lemma 6.7. Retain the notation and setting of Definition 6.6. In particular, fix P. Fix also $\vec{\eta}, \vec{\eta}_{1} \in$ $\{ \pm 1\}^{s}$. Then there is a parabolic subgroup $P^{\prime}$ satisfying the following conditions:

(1) there is a Levi decomposition $P=L N^{\prime}$;

(2) $P^{\prime}$ is $\operatorname{Ad}(G)$-conjugate to $P$;

(3) $\mathcal{I}_{P}\left(\mathcal{O} ; \vec{\eta}_{1}\right) \cong \mathcal{I}_{P^{\prime}}(\mathcal{O} ; \vec{\eta})$.

The Harish-Chandra module $\mathcal{I}_{P}(\mathcal{O} ; \vec{\eta})$ may depend on the choice of the polarization $P$ and the additional data $\vec{\eta} \in\{ \pm 1\}^{s}$. However, a result of Harish-Chandra tells us that the distribution character of a parabolically induced representation from an admissible representation of a Levi subgroup does not depend on the choice of polarization. Hence, we have the following result. 


\section{H. Matumoto and P. E. Trapa}

Proposition 6.8. We retain the setting and notation of Definition 6.6. Let $\vec{\eta}, \vec{\eta}_{1} \in\{ \pm 1\}^{s}$ and let $P$ and $P^{\prime}$ be parabolic subgroups with a common Levi subgroup $M$. Then the distribution character $\left[\mathcal{I}_{P}(\mathcal{O} ; \vec{\eta})\right]$ coincides with $\left[\mathcal{I}_{P^{\prime}}\left(\mathcal{O} ; \vec{\eta}_{1}\right)\right]$ and, hence, depends only on $\mathcal{O}$.

Proposition 6.9. Retain the setting and notation of Definition 6.6 and recall Definition 5.13. Then

$$
\operatorname{Ann}_{\mathrm{U}(\mathfrak{g})}\left(\mathcal{I}_{P}(\mathcal{O} ; \vec{\eta})\right)=I_{\mathcal{O}}
$$

Proof. The associated variety of the annihilator of a representation induced from $\pi_{M}$ is the orbit induced from the associated variety of the annihilator of $\pi_{M}$. Hence, the associated variety of the annihilator of $\mathcal{I}_{P}(\mathcal{O} ; \vec{\eta})$ is $\overline{\mathcal{O}}$. Since there is a perfect pairing between $\mathcal{I}_{P}(\mathcal{O} ; \vec{\eta})$ and $M_{\mathfrak{p}}(\delta(\mathfrak{m})-[\vec{\eta}])$, we see that the annihilator of $\mathcal{I}_{P}(\mathcal{O} ; \vec{\eta})$ is dual to $\operatorname{Ann}_{\mathrm{U}(\mathfrak{g})}\left(M_{\mathfrak{p}}(\delta(\mathfrak{m})-[\vec{\eta}])\right)$. From the argument in the proof of Proposition 5.12 we see that $\operatorname{Ann}_{\mathrm{U}(\mathfrak{g})}\left(M_{\mathfrak{p}}(\delta(\mathfrak{m})-[\vec{\eta}])\right)$ coincides with the annihilator of an irreducible generalized Verma module. Hence, $\operatorname{Ann}_{\mathrm{U}(\mathfrak{g})}\left(\mathcal{I}_{P}(\mathcal{O} ; \vec{\eta})\right)$ is a primitive ideal. Since $\mathcal{I}_{P}(\mathcal{O} ; \vec{\eta})$ has infinitesimal character $\lambda(\mathcal{O})$, the current proposition follows from Proposition 5.12.

As a consequence, we conclude that the degenerate principal series of Definition 6.6 are a source of unitary weakly unipotent representations.

Theorem 6.10. Retain the setting and notation of Definition 6.6. Each irreducible constituent of $I_{P}(\mathcal{O} ; \vec{\eta})$ is unitarizable. Moreover, if $\mathcal{O}$ is not very even (Remark 5.16), then each irreducible constituent of $I_{P}(\mathcal{O} ; \vec{\eta})$ is weakly unipotent (Definition 5.1).

Proof. We seek to show that ${ }^{n} \operatorname{Ind}_{P}^{G}\left(\left(\operatorname{det}^{\tilde{\eta} / 2}\right)^{t}\right)$ is irreducible for $0 \leqslant t<1$. Then from a standard argument using a Jantzen filtration (see, for example, [Vog84]), we can deduce that each irreducible constituent of $I_{P}(\mathcal{O} ; \vec{\eta})$ is unitarizable. From [Mat04, Corollary 5.1.2], ${ }^{n} \operatorname{Ind}_{P}^{G}(\mathbb{1})$ is irreducible; this handles the case of $t=0$. Using [Mat04, Lemma 4.1.3], we can reduce the irreducibility of ${ }^{n} \operatorname{Ind}_{P}^{G}\left(\left(\operatorname{det}^{\tilde{\eta} / 2}\right)^{t}\right)$ for $0<t<1$ to that of ${ }^{n} \operatorname{Ind}_{\bar{P}}^{\bar{G}}\left(\left(\operatorname{det}^{1 / 2}\right)^{t} A\right)$ where $\bar{G}=\mathrm{GL}\left(k_{1}+\cdots+k_{s}, \mathbb{H}\right), \bar{P}$ is a parabolic subgroup of $\bar{G}$ with a Levi part $\bar{M}=\mathrm{GL}\left(k_{1}, \mathbb{H}\right) \times \cdots \times \operatorname{GL}\left(k_{s}, \mathbb{H}\right)$, and $\operatorname{det}^{1 / 2}$ is a one-dimensional representation of $\bar{M}$ which restricts to $\operatorname{det}^{1 / 2}$ on each $\operatorname{GL}\left(k_{i}, \mathbb{H}\right)$ factor. Tensoring the one-dimensional representation $\left(\operatorname{det}^{1 / 2}\right)^{-t}$ of $\bar{G}$ with ${ }^{n} \operatorname{Ind} \bar{G}_{\bar{P}}\left(\left(\operatorname{det}^{1 / 2}\right)^{t}\right)$ gives the unitarily induced representation ${ }^{n} \operatorname{Ind} \bar{P}_{\bar{P}}(\mathbb{1})$, which is irreducible (see, for example, [Vog86]). We have thus shown that each irreducible constituent of $I_{P}(\mathcal{O} ; \vec{\eta})$ is indeed unitarizable.

From Proposition 5.12, 5.14, and 6.9, any irreducible constituent of $I_{P}(\mathcal{O} ; \vec{\eta})$ is weakly unipotent for each orbit $\mathcal{O}$ which is not very even. The proof is complete.

As preparation for Theorem 6.12, we need the following result.

Proposition 6.11. Let $Z$ be an irreducible Harish-Chandra $(\mathfrak{g}, K)$-module with an infinitesimal character in $\mathcal{P}_{G_{\mathbb{C}}}$ (with notation as in $\S 2.1$ ). Fix a Richardson orbit $\mathcal{O}$ as in Corollary 6.1 and assume that $\operatorname{Ann}_{\mathrm{U}(\mathfrak{g})}(Z) \in \operatorname{Prim}(\mathcal{O})$ (see Definition 5.3). Then $V$ is quasi P-cofinite (Definition 3.9). In particular, each $A\left(\mathcal{O}_{K}^{j}\right)$ (defined before Proposition 6.2) is quasi P-cofinite.

Proof. Let $Z$ be as in the proposition. Recall the notation of $\S 2.6$. From the translation principle, we see that there is some $[Y] \in \mathbb{B}$ such that $\operatorname{Ann}_{\mathrm{U}(\mathfrak{g})}(Y) \in \operatorname{Prim}(\mathcal{O})$ and $Y \sim Z$. From Lemma 3.10, we have only to show that $Y$ is quasi $P$-cofinite.

It follows from [McG98] that there exists a $j$ so that $Y$ and $A\left(\mathcal{O}_{K}^{j}\right)$ belong to the same cell. (McGovern actually proves that the number of cells consisting of representations annihilated by an element of $\operatorname{Prim}(\mathcal{O})$ is exactly the number $r$ of elements in $\operatorname{Irr}(\mathcal{O} \cap \mathfrak{s})=\left\{\mathcal{O}_{K}^{1}, \ldots, \mathcal{O}_{K}^{r}\right\}$.) The $r$ representations $A\left(\mathcal{O}_{K}^{j}\right)$ each have different associated varieties, and since associated varieties are constant on cells, they must belong to distinct cells. Hence, each cell of representations whose 


\section{LARGE IRREDUCIBLE CONSTITUENTS OF DEGENERATE PRINCIPAL SERIES}

annihilator is in $\operatorname{Prim}(\mathcal{O})$ contains a unique element of the form $A\left(\mathcal{O}_{K}^{j}\right)$. Let $\mathcal{C}^{j}$ denote the cell containing $A\left(\mathcal{O}_{K}^{j}\right)$.

Let $X$ be an irreducible constituent of ${ }^{n} \operatorname{Ind}_{P}^{G}(\mathbb{1})$ such that $\mathcal{O}_{K}^{j}$ is dense in an irreducible component of $\mathrm{AV}(X)$; such an $X$ exists by the computation of Proposition 3.3. Since $\mathrm{Ann}_{\mathrm{U}(\mathfrak{g})}(X)=$ $\operatorname{Ann}_{\mathrm{U}(\mathfrak{g})}\left({ }^{n} \operatorname{Ind}_{P}^{G}(\mathbb{1})\right) \in \operatorname{Prim}(\mathcal{O})$, the discussion of the previous paragraph implies that $X$ is an element of $\mathcal{C}^{j}$. From Lemma 3.10, we conclude $Y$ is quasi $P$-cofinite. The proposition follows.

Theorem 6.10 showed us that the degenerate principal series of Definition 6.6 were a source of weakly unipotent representations attached to $\mathcal{O}$. The following theorem implies that all such unipotent representations are obtained this way (apart from the very even case).

Theorem 6.12. Retain the notation of Definition 6.6 and Proposition 6.2.

(1) In the notation introduced at the end of $\S 2.4$,

$$
\mathcal{I}_{P}(\mathcal{O} ; \vec{\eta}) \approx \bigoplus_{j=1}^{r} A\left(\mathcal{O}_{K}^{j}\right) .
$$

That is, $\mathcal{I}_{P}(\mathcal{O} ; \vec{\eta})$ has a composition series in which each $A\left(\mathcal{O}_{K}^{j}\right)$ appears exactly once. Moreover, $A\left(\mathcal{O}_{K}^{1}\right), \ldots, A\left(\mathcal{O}_{K}^{r}\right)$ exhaust the irreducible constituent of maximal Gelfand-Kirillov dimension.

(2) Suppose that $X$ is an integral weakly unipotent representation attached to $\mathcal{O}$ (Definition 5.1). Assume that $\mathcal{O}$ is not very even (Remark 5.16). Then there exists $j$ such that

$$
X \cong A\left(\mathcal{O}_{K}^{j}\right) .
$$

(3) Suppose that $X$ is a distinguished unipotent representation attached to $\mathcal{O}$ (Definition 5.1). Then there exists $j$ such that

$$
X \cong A\left(\mathcal{O}_{K}^{j}\right) .
$$

Proof. Suppose that $X$ is any representation whose infinitesimal character lies in $\mathcal{P}_{G_{\mathbb{C}}}$ and whose annihilator lies in $\operatorname{Prim}(\mathcal{O})$. Then Proposition 6.11 implies that there is some finite-dimensional representation $F$ such that $X$ is isomorphic to an irreducible constituent of ${ }^{n} \operatorname{Ind}_{P}^{G}(F)$. However, at the infinitesimal character $\lambda(\mathcal{O}),{ }^{n} \operatorname{Ind}_{P}^{G}(F)$ must be of the form $\mathcal{I}_{P}(\mathcal{O} ; \vec{\eta})$ given in Definition 6.6, and we know from Proposition 6.8 that all such representations have the same distribution character.

Take $X=A\left(\mathcal{O}_{K}^{j}\right)$ in the preceding paragraph. We conclude that each $A\left(\mathcal{O}_{K}^{j}\right)$ appears as a composition factor of each $\mathcal{I}_{P}(\mathcal{O} ; \vec{\eta})$. Corollary 3.2 says that each $A\left(\mathcal{O}_{K}^{j}\right)$ appears exactly once. Propositions 3.3(1) and 6.2 and the additivity of the associated cycle on modules of the same maximal Gelfand-Kirillov dimension shows there can be no other constituents of maximal GelfandKirillov dimension. We thus obtain the first assertion of the current proposition. Now parts (2) and (3) follow from part (1) and the previous paragraph.

Using the translation principle, it turns out that we deduce from the 'most singular' case of Theorem 6.12 results about other less singular infinitesimal characters. This is the content of Proposition 6.13. To formulate it, we need further notation.

For each $1 \leqslant j \leqslant r$, fix a $\theta$-stable parabolic subalgebra $\mathfrak{q}^{j}=\mathfrak{l}^{j} \oplus \mathfrak{u}^{j}$ as in Proposition 4.9. Since $\mathfrak{p}$ and $\mathfrak{q}^{j}$ are $\operatorname{Ad}\left(G_{\mathbb{C}}\right)$-conjugate, we fix $g^{j} \in G_{\mathbb{C}}$ such that $\operatorname{Ad}\left(g^{j}\right) \mathfrak{p}=\mathfrak{q}^{j}$. Set $\mathfrak{h}^{j}=\operatorname{Ad}\left(g^{j}\right) \mathfrak{h}$. Let $\chi: M_{\mathbb{C}} \rightarrow \mathbb{C}^{\times}$be a holomorphic one-dimensional representation. We define $\chi^{j}=\chi \circ \operatorname{Ad}\left(g^{j}\right)^{-1}$, a holomorphic character of the analytic subgroup $L_{\mathbb{C}}^{j}$ of $G_{\mathbb{C}}$ corresponding to $\mathfrak{l}^{j}$.

Write $X_{P}$ for the complexified generalized flag variety $G_{\mathbb{C}} / P_{\mathbb{C}}$. For each $1 \leqslant j \leqslant r$, let $\mathcal{V}^{j}$ denote the (open) $G$-orbit in $G_{\mathbb{C}} / P_{\mathbb{C}}$ containing $\mathfrak{q}^{j}$. Proposition 4.9 implies that $\mathcal{V}^{1}, \ldots, \mathcal{V}^{r}$ exhaust all of the good open $G$-orbits in $X_{P}$. 


\section{H. Matumoto and P. E. Trapa}

For $X \in \mathfrak{m}$, define

$$
\delta_{P}(X)=\frac{1}{2} \operatorname{tr}\left(\left.\operatorname{ad}_{\mathfrak{g}}(X)\right|_{\mathfrak{n}}\right) .
$$

Then $\delta$ is a one-dimensional representation of $\mathfrak{m}$, and $2 n \delta$ lifts to a holomorphic group homomorphism $\xi_{2 n \delta_{P}}: M_{\mathbb{C}} \rightarrow \mathbb{C}^{\times}$for any $n \in \mathbb{Z}$. As usual, we may regard $\xi_{2 \delta}$ as a one-dimensional representation of $P$. With this notation $\xi_{0}=\xi_{0 \delta_{P}}$ is the trivial representation of $P$.

Let $\omega_{P}$ be the canonical line bundle on $X_{P}$ and let $\mathcal{L}_{\chi}$ denote the homogeneous holomorphic line bundle on $X_{P}$ associated to the character $\chi$. So, for instance, $\omega_{P}=\mathcal{L}_{\xi_{2 \delta_{P}}}$. For a character $\chi$ and $1 \leqslant j \leqslant r$, we may consider the derived functor module

$$
A_{\mathcal{V}^{j}}(\chi)=A_{\mathfrak{q}^{j}}\left(\chi^{j}\right)=H^{\operatorname{dim}\left(\mathfrak{k} \cap \mathfrak{u}^{j}\right)}\left(\mathcal{V}, \mathcal{L}_{\chi} \otimes \omega_{P}\right)_{K \text {-finite }}
$$

Fix a $\theta$-stable Cartan subalgebra $\mathfrak{h}_{0}$ of $\mathfrak{g}_{0}$ and a Borel subalgebra $\mathfrak{b}$ of $\mathfrak{g}$ such that $\mathfrak{h} \subseteq \mathfrak{b} \subseteq \mathfrak{p}$ and $\mathfrak{h} \subseteq \mathfrak{m}$. Let $\Delta^{+}(\mathfrak{g}, \mathfrak{h})$ denote the positive system of $\Delta(\mathfrak{g}, \mathfrak{h})$ corresponding to $\mathfrak{b}$.

Let $\chi$ be a holomorphic character of $M_{\mathbb{C}}$ and write $d \chi$ for the differential of $\chi$. In addition, write $d \chi$ for the restriction of $d \chi: \mathfrak{m} \rightarrow \mathbb{C}$ to $\mathfrak{h}$. We call $\chi P$-good (respectively, $P$-weakly fair) if $\left\langle d \chi+\rho, \alpha^{\vee}\right\rangle>0$ (respectively, $\left\langle d \chi+\delta_{P}, \alpha^{\vee}\right\rangle \geqslant 0$ ) for all $\alpha \in \Delta^{+}(\mathfrak{g}, \mathfrak{h})$. If $\chi$ is $P$-good (respectively, $P$-weakly fair), then $A_{\mathcal{V}^{j}}(\chi)$ is in the good range (respectively, the weakly fair range) in the sense of $[\operatorname{Vog} 88]$.

Proposition 6.13. Retain the notation introduced just before the proposition (especially that of (6.4).) In particular, let $\chi$ be a $P$-weakly fair holomorphic character of $M_{\mathbb{C}}$. Let $\mathcal{I}_{P}(\chi)$ denote the degenerate principal series representation ${ }^{n} \operatorname{Ind}_{P}^{G}\left(\mathbb{C}_{\delta_{P}} \otimes \chi\right)$. Then (in the notation introduced at the end of $\S 2.4$ ),

$$
\mathcal{I}_{P}(\chi) \approx \bigoplus_{j=1}^{r} A_{\mathcal{V}^{j}}(\chi) .
$$

Proof. First, we remark that for any $\vec{\eta} \in\{ \pm 1\}^{s}$, $\operatorname{det}^{\tilde{\eta} / 2} \otimes \xi_{-\delta_{P}}$ can be extended to a holomorphic character of $M_{\mathbb{C}}($ say $\chi(\vec{\eta}))$. Then we easily see $\mathcal{I}_{P}(\mathcal{O}, \vec{\eta})=\mathcal{I}_{P}(\chi(\vec{\eta}))$. Moreover, if we appropriately choose $\vec{\eta} \in\{ \pm 1\}^{s}$, then $\operatorname{det}^{\tilde{\eta} / 2} \otimes \mathbb{C}_{-\delta_{P}}$ is $P$-weakly fair. Hereafter we fix such a choice for $\vec{\eta}$. We need the following lemma, which is well known (see, for example, [Vog88] and [Vog90]).

Lemma 6.14. For each $P$-weakly fair $\chi$, we have $T_{d \chi+\rho}^{d \chi(\vec{\eta})+\rho}\left(A_{\mathcal{V}^{j}}(\chi)\right)=A_{\mathcal{V}^{j}}(\chi(\vec{\eta}))=A\left(\mathcal{O}_{K}^{j}\right)$. Here, $T_{d \chi^{\prime}+\rho}^{d \chi+\rho}$ means the translation functor from $d \chi+\rho$ to $d \chi(\vec{\eta})+\rho$ defined by tensoring with the irreducible finite-dimensional representation with lowest weight $d \chi(\vec{\eta})-d \chi$.

Continuation of the proof of Proposition 6.13. From Corollary 3.2 and arguing as in the proof of Theorem 6.12, we have only to show that $A_{\mathcal{V}^{j}}(\chi)$ appears in $\mathcal{I}_{P}(\chi)$ as an irreducible constituent. Let $Z$ be an irreducible constituent of $\mathcal{I}_{P}(\chi)$. From the proof of Proposition 6.11, we know that $Z$ lies in the same cell as some $A_{\mathcal{V}^{j}}(\chi)$. Thus, $\operatorname{AV}(Z)=\overline{\mathcal{O}_{K}^{j}}$ for some $j$. Put $Z^{\prime}=T_{\underline{d \chi+\rho}}^{d \chi(\vec{\eta})+\rho}(Z)$. Since the differential of $\operatorname{det}^{\tilde{\eta} / 2}$ is $[\vec{\eta}]$, we have $d \chi(\vec{\eta})+\rho=\delta(\mathfrak{m})+[\vec{\eta}]$. Since $\operatorname{AV}\left(Z^{\prime}\right)=\overline{\mathcal{O}_{K}^{j}}$, we conclude $\operatorname{Ann}_{\mathrm{U}(\mathfrak{g})}\left(Z^{\prime}\right) \in \operatorname{Dist}_{G_{\mathbb{C}}}(\mathcal{O})$. Theorem 6.12(2) implies that $Z^{\prime} \cong A\left(\mathcal{O}_{K}^{j}\right)$. On the other hand, from Lemma 6.14, we have $A\left(\mathcal{O}_{K}^{j}\right)=T_{d \chi+\rho}^{d \chi(\vec{\eta})+\rho}\left(A_{\mathcal{V}^{j}}(\chi)\right)$. Therefore, from [Vog90, Proposition 7.7], we have $Z \cong A_{\mathcal{V}^{j}}(\chi)$.

As a corollary of the above theorem, we obtain a nice characterization of constituents of maximal Gelfand-Kirillov dimension in integral degenerate principal series.

Corollary 6.15. Let $P$ be a parabolic subgroup of $G$ and let $X$ be a representation of $G$ parabolically induced from a one-dimensional representation of $P$. Assume that the infinitesimal character of $X$ lies in $\mathcal{P}_{G_{\mathbb{C}}}$. Then any irreducible constituent of $X$ of the maximal Gelfand-Kirillov dimension is a derived functor module in the weakly fair range. 


\section{LARGE IRREDUCIBLE CONSTITUENTS OF DEGENERATE PRINCIPAL SERIES}

Proof. We may write $X$ as $\mathcal{I}_{P}(\chi)$ for some holomorphic character. We easily see that there is some parabolic subgroup $P^{\prime}$ whose Levi part coincides with that of $P$ such that $\chi$ is $P^{\prime}$-weakly fair. Since $\mathcal{I}_{P}(\chi)$ and $\mathcal{I}_{P^{\prime}}(\chi)$ have the same distribution character (Proposition 6.8), the corollary follows from Proposition 6.13.

Remark 6.16. In the proof of Corollary $6.15, P^{\prime}$ is not necessarily $\operatorname{Ad}(G)$-conjugate to $P$. So, although an irreducible constituent of $X$ in Corollary 6.15 can be written in the form $A_{\mathfrak{q}}(\lambda), \mathfrak{q}$ is not necessarily $\operatorname{Ad}\left(G_{\mathbb{C}}\right)$-conjugate to $\mathfrak{p}$. This means that $A_{\mathfrak{q}}(\lambda)$ is not necessarily attached to an open $G$-orbit in the complexified generalized flag manifold $X_{P}$.

To conclude this section, we turn our attention to edge-of-wedge-type embeddings. We begin with the following lemma in the general setting. In its statement, we let $M^{h}$ denote the Hermitian dual of a Harish-Chandra module $M$ (see e.g., [KV95, § VI.2]).

Lemma 6.17. Let $G$ be a real reductive group. Let $I$ be a Harish-Chandra $(\mathfrak{g}, K)$-module and let $\Psi: I^{h} \rightarrow I$ be a $(\mathfrak{g}, K)$-homomorphism. We invoke the following three assumptions:

(a1) $\operatorname{Dim}(\operatorname{Kernel}(\Psi))<\operatorname{Dim}\left(I^{h}\right)$;

(a2) let $V$ be an arbitrary irreducible constituent of $I$ such that $\operatorname{Dim}(V)=\operatorname{Dim}(I)$, then $V^{h} \cong V$ and the multiplicity of $V$ in $I$ is one;

(a3) let $W$ be any submodule of $I$, then $\operatorname{Dim}(W)=\operatorname{Dim}(I)$.

Let $\operatorname{Socle}(I)$ denote the largest semisimple submodule of $I$. Then:

(1) $\operatorname{Socle}(I)=\Psi\left(I^{h}\right)$;

(2) $\operatorname{Dim}(I / \operatorname{Socle}(I))<\operatorname{Dim}(I)$.

In particular, any irreducible constituent $Y$ of $I$ such that $\operatorname{Dim}(Y)=\operatorname{Dim}(I)$ is a submodule of $I$.

Proof. Let $Y$ be any irreducible submodule of $I$. From assumption (a3), we have $\operatorname{Dim}(Y)=\operatorname{Dim}(I)$. From assumption (a1), we see $\Psi\left(I^{h}\right)$ contains an irreducible constituent isomorphic to $Y$. However, since the multiplicity of $Y$ in $I$ is one (by assumption (a2)), it follows that indeed $Y$ is in the image $\Psi\left(I^{h}\right)$. Hence, $\operatorname{Socle}(I) \subseteq \Psi\left(I^{h}\right)$.

In order to show assertion (1), we thus have only to show that $\Psi\left(I^{h}\right) \cong I^{h} / \operatorname{Kernel}(\Psi)$ is semi simple. Let $W$ be any irreducible quotient of $I^{h} / \operatorname{Kernel}(\Psi)$. Then $W$ is also a quotient of $I^{h}$ and so $W^{h}$ is realized as a submodule of $I$. According to assumption (a2), $W \simeq W^{h}$, and thus from the previous paragraph, we conclude that the image $\Psi\left(I^{h}\right)$ contains a submodule isomorphic to $W$. By assumption (a2), $W$ has multiplicity one in $\Psi\left(I^{h}\right)$. We conclude that any irreducible quotient $W$ of $\Psi\left(I^{h}\right)$ is also a submodule. Thus, $\Psi\left(I^{h}\right)$ is semisimple and assertion (1) follows. Assertion (2) follows from assumption (a1) and assertion (1).

We consider the following special case. Fix a parabolic subgroup $P$ of $G$ with a $\theta$-stable Levi part $M$. Consider the unnormalized generalized Verma modules as above,

$$
{ }^{u} M_{\mathfrak{p}}(\mu)=\mathrm{U}(\mathfrak{g}) \otimes_{\mathrm{U}(\mathfrak{p})} \mathbb{C}_{\mu} .
$$

Let $\chi$ be a holomorphic character of $M_{\mathbb{C}}$. Recall that there is a perfect pairing between ${ }^{u} M_{\mathfrak{p}}\left(-2 \delta_{P}-d \chi\right)$ and $\mathcal{I}_{P}(\chi)$ (where $\mathcal{I}(\chi)$ is defined in Proposition 6.13).

The following is an edge-of-wedge embedding result for $\operatorname{Sp}(p, q)$.

Theorem 6.18. Let $G=\operatorname{Sp}(p, q)$ and fix a parabolic subgroup $P=M N$ with

$$
M=\mathrm{GL}\left(k_{1}, \mathbb{H}\right) \times \cdots \times \mathrm{GL}\left(k_{s}, \mathbb{H}\right) \times \mathrm{Sp}\left(p^{\prime}, q^{\prime}\right) .
$$

For each positive integer $\ell$, let $m_{M}(\ell)$ be the number of $i$ between 1 and $s$ such that $k_{i}=\ell$. Assume the following. 


\section{H. Matumoto and P. E. Trapa}

(C1) For each $\ell>p^{\prime}+q^{\prime}, m_{M}(\ell)$ is even.

Let $\chi$ be a $P$-weakly fair holomorphic character of $M_{\mathbb{C}}$. Then we have the following.

(a) There is an embedding of generalized Verma modules

$$
\psi_{\chi}:{ }^{u} M_{\mathfrak{p}}\left(-2 \delta_{P}-d \chi\right) \hookrightarrow{ }^{u} M_{\mathfrak{p}}(d \chi) .
$$

This induces an intertwining operator

$$
\Psi_{\chi}: \mathcal{I}_{P}\left(\chi^{-1} \otimes \xi_{-2 \delta_{P}}\right) \rightarrow \mathcal{I}_{P}(\chi)
$$

(cf. [CS90, § 2]).

(b) We have $\operatorname{Socle}\left(\mathcal{I}_{P}(\chi)\right)=\Psi_{\chi}\left(\mathcal{I}_{P}\left(\chi^{-1} \otimes \xi_{-2 \delta_{P}}\right)\right.$ ). Moreover, $\operatorname{Socle}\left(\mathcal{I}_{P}(\chi)\right) \approx \mathcal{I}_{P}(\chi)$ (with notation as in the end of $\S 2.4)$.

(c) We have that the decomposition of Proposition 6.13 is indeed the socle,

$$
\operatorname{Socle}\left(\mathcal{I}_{P}(\chi)\right)=\bigoplus_{j=1}^{r} A_{\mathcal{V}^{j}}(\chi) \text {. }
$$

Proof. Set $I=\mathcal{I}_{P}(\chi)$. Then $I^{h}=\mathcal{I}\left(\chi^{-1} \otimes \xi_{-2 \delta_{P}}\right)$. Part (a) is obtained in [Mat03, Theorem 5.1.2]. (This is where assumption (C1) is needed.) Thus, to establish the theorem, we need only verify that the assumptions (a1)-(a3) of Lemma 6.17 are satisfied. Since $\operatorname{Kernel}(\Psi)$ has a perfect pairing with ${ }^{u} M_{\mathfrak{p}}(d \chi) /{ }^{u} M_{\mathfrak{p}}\left(-2 \delta_{P}-d \chi\right)$ and $\operatorname{Dim}\left({ }^{u} M_{\mathfrak{p}}(d \chi) / M_{\mathfrak{p}}\left(-2 \delta_{P}-d \chi\right)\right)<\operatorname{Dim}\left(M_{\mathfrak{p}}\left(-2 \delta_{P}-d \chi\right)\right)=\operatorname{Dim}(I)$, we have $\operatorname{Dim}(\operatorname{Kernel}(\Psi))<\operatorname{Dim}(I)$. This is assumption (a1).

Condition (a2) follows from Proposition 6.13. Moreover, since ${ }^{u} M_{\mathfrak{p}}\left(-2 \delta_{P}-d \chi\right)$ is irreducible, any submodule of $I$ also has a perfect pairing with $M_{\mathfrak{p}}\left(-2 \delta_{P}-d \chi\right)$. This implies that any submodule of $I$ has the same Gelfand-Kirillov dimension as $I$. This is assumption (a3) and the proof is complete.

The following result for $\mathrm{SO}^{*}(2 n)$ is obtained in a similar way.

Theorem 6.19. Let $G=\mathrm{SO}^{*}(2 n)$ and let $P=M N$ be a parabolic subgroup with

$$
M=\mathrm{GL}\left(k_{1}, \mathbb{H}\right) \times \cdots \times \mathrm{GL}\left(k_{s}, \mathbb{H}\right) \times \mathrm{SO}^{*}\left(2 n^{\prime}\right) .
$$

For each positive integer $\ell$, let $m_{M}(\ell)$ be the number of $i$ between 1 and $s$ such that $k_{i}=\ell$. Assume the following.

(C2) For each $\ell \leqslant n^{\prime}, m_{M}(\ell)$ is even.

Let $\chi$ be a $P$-weakly fair holomorphic character of $M_{\mathbb{C}}$. Then, the conclusions (a)-(c) of Theorem 6.18 hold in the present context.

\section{The group $\mathrm{U}(m, n)$}

For $\mathrm{U}(m, n)$, Theorems 7.3 and 7.4 below establish results analogous to those proved for $\operatorname{Sp}(p, q)$ in Corollary 6.15 and Theorem 6.18. It is possible to imitate the approach of $\S 6$ in the context of $\mathrm{U}(p, q)$ and, in particular, it is possible to define a version of the representation $\mathcal{I}_{P}(\mathcal{O})$ considered in Definition 6.6. However, such a $\mathcal{I}_{P}(\mathcal{O})$ is not necessarily a class one degenerate principal series. Moreover, the argument leading to the proof of Corollary 6.15 cannot be imitated for $\mathrm{U}(m, n)$. In this section we instead prove the main results of Theorems 7.3 and 7.4 in a different way.

Henceforth in this section we fix $G=\mathrm{U}(m, n)$. We fix a maximal compact subgroup $K \simeq \mathrm{U}(m) \times$ $\mathrm{U}(n)$ of $G$ and let $\theta$ denote the corresponding Cartan involution. As usual, we let $\mathfrak{g}$ (respectively, $\mathfrak{k}$ ) denote the complexified Lie algebra of $G$ (respectively, $K)$; so $\mathfrak{g} \cong \mathfrak{g l}(m+n, \mathbb{C})$ and $\mathfrak{k} \cong \mathfrak{g l}(m, \mathbb{C}) \oplus$ $\mathfrak{g l}(n, \mathbb{C})$. 


\section{LARGE IRREDUCIBLE CONSTITUENTS OF DEGENERATE PRINCIPAL SERIES}

Let $P$ be a parabolic subgroup of $G$ and choose a $\theta$-stable maximally split Cartan subgroup ${ }^{s} H$ of $G$ such that ${ }^{s} H \subseteq P$. (We remark that all Cartan subgroups of $G$ are connected.) Let $M$ denote the $\theta$-stable Levi part of $P$ (and so ${ }^{s} H \subseteq M$ ), and write $N$ for the nilradical of $P$. We let ${ }^{s} \mathfrak{h}$ the complexified Lie algebra of ${ }^{s} H$.

Fix a Borel subalgebra $\mathfrak{b}$ such that ${ }^{s} \mathfrak{h} \subseteq \mathfrak{b} \subseteq \mathfrak{p}$. For simplicity, write $\Delta$ for $\Delta\left(\mathfrak{g},{ }^{s} \mathfrak{h}\right)$. We let $\Delta^{+}$ denote the system of positive roots corresponding to $\mathfrak{b}$ and let $\Pi$ denote the corresponding basis of $\Delta$. We choose an orthonormal basis $e_{1}, \ldots, e_{m+n}$ of ${ }^{s} \mathfrak{h}^{*}$ so that

$$
\begin{gathered}
\Delta=\left\{e_{i}-e_{j} \mid 1 \leqslant i, j \leqslant m+n, i \neq j\right\}, \\
\Pi=\left\{e_{1}-e_{2}, \ldots, e_{m+n-1}-e_{m+n}\right\} .
\end{gathered}
$$

We modify our original choice of $\mathfrak{b}$ appropriately so that $\theta\left(e_{i}\right)=e_{m+n-i+1}$ for all $1 \leqslant i \leqslant \min \{m, n\}$ and $\theta\left(e_{i}\right)=e_{i}$ for all $\min \{m, n\}<i<m+n-\min \{m, n\}$.

Let $\kappa=\left(k_{1}, \ldots, k_{s}\right)$ be a finite sequence of positive integers such that

$$
k=k_{1}+\cdots+k_{s} \leqslant \min \{m, n\} .
$$

Put $k_{i}^{*}=k_{1}+\cdots+k_{i}$ for $1 \leqslant i \leqslant s$ and $k_{0}^{*}=0$. Hence, $k_{s}^{*}=k$. Set $m^{\prime}=m-k$ and $n^{\prime}=n-k$. Define a subset $S(\kappa)$ of $\Pi$ as follows,

$$
S(\kappa)=\Pi-\left\{e_{k_{i}^{*}}-e_{k_{i}^{*}+1}, e_{m+n-k_{i}^{*}}-e_{m+n-k_{i}^{*}+1} \mid 1 \leqslant i \leqslant s\right\} .
$$

We easily see that there exists some $\kappa=\left(k_{1}, \ldots, k_{s}\right)$ as above such that $\mathfrak{p}$ is the standard parabolic subalgebra containing the Borel subalgebra $\mathfrak{b}$ corresponding to $S(\kappa)$, i.e. such that $S(\kappa)$ is a basis of $\Delta\left(\mathfrak{m},{ }^{s} \mathfrak{h}\right) \cap \Delta^{+}$.

Formally, we let $\mathrm{U}(0,0)$ denote the trivial group $\{1\}$ and we let $\operatorname{GL}(\kappa, \mathbb{C})$ denote the product $\operatorname{GL}\left(k_{1}, \mathbb{C}\right) \times \cdots \times \operatorname{GL}\left(k_{s}, \mathbb{C}\right)$. Then,

$$
M_{\kappa} \cong \mathrm{GL}(\kappa, \mathbb{C}) \times \mathrm{U}\left(m^{\prime}, n^{\prime}\right),
$$

and $\mathrm{GL}(\kappa, \mathbb{C})$ and $\mathrm{U}\left(m^{\prime}, n^{\prime}\right)$ can be identified with subgroups of $M$. The Cartan involution $\theta$ induces Cartan involutions on $M, \mathrm{GL}(\kappa, \mathbb{C}), \mathrm{U}\left(m^{\prime}, n^{\prime}\right)$ and we denote them by the same letter $\theta$.

Next we consider induced representations from characters of parabolic subgroups. Let $\mu$ and $\nu$ be complex numbers such that $\mu+\nu \in \mathbb{Z}$. We define a one-dimensional representation $\left(\eta_{\mu, \nu}^{k}, \mathbb{C}_{\mu, \nu}^{k}\right)$ of $\mathrm{GL}(k, \mathbb{C})$ as follows:

$$
\eta_{\mu, \nu}^{k}(g)=\operatorname{det}(g)^{\mu} \overline{\operatorname{det}(g)}^{-\nu} \quad(g \in \mathrm{GL}(k, \mathbb{C})) .
$$

For $h \in \mathbb{Z}$, we define a one-dimensional representation $\left(\eta_{h}^{p, q}, \mathbb{C}_{h}^{p, q}\right)$ of $\mathrm{U}(p, q)$ as follows:

$$
\eta_{h}^{p, q}(g)=\operatorname{det}(g)^{h} \quad(g \in \mathrm{U}(p, q)) .
$$

Let $\mathbf{u}=\left(u_{1}, \ldots, u_{s}\right)$ and $\mathbf{v}=\left(v_{1}, \ldots, v_{s}\right)$ be sequences of complex numbers such that $u_{i}+v_{i} \in \mathbb{Z}$ for all $1 \leqslant i \leqslant s$. Let $h \in \mathbb{Z}$. We define a one-dimensional representation $\mathbb{C}_{\mathbf{u} ; h ; \mathbf{v}}^{\kappa}$ of $\operatorname{GL}(k, \mathbb{C}) \times \mathrm{U}(m-$ $k, n-k)$ by

$$
\mathbb{C}_{\mathbf{u} ; h ; \mathbf{v}}^{\kappa}=\mathbb{C}_{u_{1}, v_{1}}^{k_{1}} \otimes \cdots \otimes \mathbb{C}_{u_{s}, v_{s}}^{k_{s}} \otimes \mathbb{C}_{h}^{m-k, n-k} .
$$

When we identify $M$ with $\mathrm{GL}(\kappa, \mathbb{C}) \times \mathrm{U}(m-k, n-k)$, there are ambiguities arising from automorphisms of $\operatorname{GL}(\kappa, \mathbb{C})$, i.e. any identification we choose may be twisted by complex conjugation. We choose the identification so that the differential of the restriction of $\mathbb{C}_{\mathbf{u}, h, \mathbf{v}}^{\kappa}$ to ${ }^{s} H$ is

$$
\sum_{j=1}^{s} \sum_{i=1}^{k_{j}}\left[u_{j} e_{k_{j-1}^{*}+i}+v_{j} e_{m+n-k_{j-1}^{*}-i+1} \in{ }^{s} \mathfrak{h}^{*}\right]+h \sum_{\ell=1}^{m+n-2 k} e_{k+\ell},
$$




\section{H. Matumoto and P. E. Trapa}

i.e. we assign $e_{1}, \ldots, e_{k}$ (respectively, $e_{m+n-k+1}, \ldots, e_{m+n}$ ) to the holomorphic (respectively, antiholomorphic) part. We consider the following degenerate principal series representation of $G$,

$$
\mathcal{I}_{P}[\mathbf{u} ; h ; \mathbf{v}]={ }^{n} \operatorname{Ind}_{P}^{G}\left(\mathbb{C}_{\mathbf{u} ; h ; \mathbf{v}}^{\kappa}\right) .
$$

We let $\delta_{m, n}^{\kappa}$ denote the element of $\mathbb{C}^{s}$ whose $i$ th entry is $\left(m+n-k_{i}^{*}+k_{i}\right) / 2$. If $m=n=k$, we regard $\mathbb{C}_{h}^{0,0}$ as the trivial representation of the trivial group. In this case $\mathcal{I}_{P}[\mathbf{u} ; h ; \mathbf{v}]$ does not depend on $h$.

We let $J(\mathbf{u}, h, \mathbf{v})$ denote the annihilator of $\mathcal{I}_{P}[\mathbf{u} ; h ; \mathbf{v}]$ in the universal enveloping algebra $\mathrm{U}(\mathfrak{g})$.

We define a normalized generalized Verma module as follows. Let $\mathfrak{p}$ be a parabolic subalgebra of $\mathfrak{g}=\mathfrak{g l}(m+n)$ with Levi decomposition $\mathfrak{p}=\mathfrak{l}+\mathfrak{n}$. We define a one-dimensional representation $-\rho_{\mathfrak{p}}$ of $\mathfrak{l}$ by $-\rho_{\mathfrak{p}}(X)=\frac{1}{2}\left(\left.\operatorname{ad}(X)\right|_{\mathfrak{n}}\right)(X \in \mathfrak{l})$. For a one-dimensional representation $\xi$ of $\mathfrak{l}$ we extend $\xi \otimes-\rho_{\mathfrak{p}}$ to a one-dimensional representation of $\mathfrak{p}$ as usual and define ${ }^{n} M_{\mathfrak{p}}(\xi)=\mathrm{U}(\mathfrak{g}) \otimes_{\mathrm{U}(\mathfrak{p})}\left(\xi \otimes-\rho_{\mathfrak{p}}\right)$.

We define a weight on ${ }^{s} \mathfrak{h}$ by

$$
\xi(\mathbf{u}, h, \mathbf{v})=\sum_{i=1}^{s} u_{i}\left(\sum_{j=1}^{k_{i}} e_{k_{i-1}^{*}+j}\right)+h \sum_{j=1}^{m+n-2 k} e_{k+j}+\sum_{i=1}^{s} v_{s-i}\left(\sum_{j=1}^{k_{i}} e_{m+n-k+k_{i-1}^{*}+j}\right) .
$$

Obviously, $\xi(\mathbf{u}, h, \mathbf{v})$ can be extended to a one-dimensional representation of $\mathfrak{l}$. We let $\overline{\mathfrak{p}}_{\kappa}$ denote the opposite parabolic subalgebra to $\mathfrak{p}_{\kappa}$.

Recall that ${ }^{n} \mathcal{I}_{P}[\mathbf{u} ; h ; \mathbf{v}]$ and ${ }^{n} M_{\mathfrak{p}_{\kappa}}(-\xi(\mathbf{u}, h, \mathbf{v}))$ admit perfect pairing; ${ }^{n} M_{\overline{\mathfrak{p}}_{\kappa}}(\xi(\mathbf{u}, h, \mathbf{v}))$ and ${ }^{n} M_{\mathfrak{p}_{\kappa}}(-\xi(\mathbf{u}, h, \mathbf{v}))$ also admit a perfect pairing. Hence, we have the following lemma.

Lemma 7.1. The annihilator $J(\mathbf{u}, h, \mathbf{v})$ coincides with the annihilator of ${ }^{n} M_{\overline{\mathfrak{p}}_{\kappa}}(\xi(\mathbf{u}, h, \mathbf{v}))$.

Let $\mathbf{c}=\left(c_{1}, \ldots, c_{\ell}\right)$ be a sequence of nonnegative integers such that $c_{1}+\cdots+c_{\ell}=m+n$. Put $c_{i}^{*}=c_{1}+\cdots+c_{i}$ and $c_{0}^{*}=0$. We define a subset $S[\mathbf{c}]$ of $\Pi$ as follows,

$$
S[\mathbf{c}]=\Pi-\left\{e_{c_{i}^{*}}-e_{c_{i}^{*}+1} \mid 1 \leqslant i \leqslant \ell\right\} .
$$

We let $\mathfrak{p}(\mathbf{c})$ denote the standard parabolic subalgebra of $\mathfrak{g}$ associated to $S[\mathbf{c}]$. Under an appropriate realization of $\mathfrak{g}$ as $\mathfrak{g l}(m+n, \mathbb{C}), \mathfrak{p}(\mathbf{c})$ is the block-upper-triangular parabolic subalgebra of $\mathfrak{g l}(m+n, \mathbb{C})$ with blocks of sizes $c_{1}, \ldots, c_{\ell}$ along the diagonal. If $c_{i}=0$ for some $i$, 'a block of size 0 ' means nothing: we simply neglect it. We let $\overline{\mathfrak{p}}(\mathbf{c})$ denote the parabolic subalgebra opposite to $\mathfrak{p}(\mathbf{c})$. For a sequence $\mathbf{h}=\left(h_{1}, \ldots, h_{\ell}\right)$ of complex numbers, we define a weight on ${ }^{s} \mathfrak{h}$ as follows:

$$
\xi(\mathbf{h})=\sum_{i=1}^{\ell} h_{i}\left(\sum_{j=1}^{c_{i}} e_{c_{i-1}^{*}+j}\right)
$$

Fix a degenerate principal series representation ${ }^{n} \mathcal{I}_{P}[\mathbf{u} ; h ; \mathbf{v}]$ with an integral infinitesimal character. In particular, $\mathbf{u}$ and $\mathbf{v}$ are fixed so that $\mathbf{u}-\delta_{m, n}^{\kappa}, \mathbf{v}+\delta_{m, n}^{\kappa} \in \mathbb{Z}^{s}$. We define $\mathbf{h}=\left(h_{1}, \ldots, h_{2 s+1}\right)$ as follows:

$$
\begin{gathered}
h_{1}=h, \\
h_{2 i}=u_{s-i+1} \quad(1 \leqslant i \leqslant s), \\
h_{2 i+1}=v_{i} \quad(1 \leqslant i \leqslant s) .
\end{gathered}
$$

We also define a sequence of positive integer $\mathbf{c}$ by

$$
\begin{aligned}
c_{1} & =m+n-2 k, \\
c_{2 i}=c_{2 i+1} & =k_{s-i+1} \quad(1 \leqslant i \leqslant s) .
\end{aligned}
$$

Finally, we define

$$
\Xi(\mathbf{u}, h, \mathbf{v})=\left\{\tau \in \mathfrak{S}_{2 s+1} \mid h_{\tau(1)} \geqslant \cdots \geqslant h_{\tau(2 s+1)}\right\}
$$




\section{LARGE IRREDUCIBLE CONSTITUENTS OF DEGENERATE PRINCIPAL SERIES}

Obviously $\Xi(\mathbf{u}, h, \mathbf{v})$ is nonempty. For $\tau \in \mathfrak{S}_{2 s+1}$, put $\mathbf{c}^{\tau}=\left(c_{\tau(1)}, \ldots, c_{\tau(2 s+1)}\right)$ and $\mathbf{h}^{\tau}=\left(h_{\tau(1)}, \ldots, h_{\tau(2 s+1)}\right)$. From [BJ77, 4.10 Corollar] and Lemma 7.1, we have the following.

Proposition 7.2. If $\tau \in \Xi(\mathbf{u}, h, \mathbf{v})$, then $J(\mathbf{u}, h, \mathbf{v})$ coincides with the annihilator of ${ }^{n} M_{\overline{\mathfrak{p}}\left(\mathbf{c}^{\tau}\right)}\left(\xi\left(\mathbf{h}^{\tau}\right)\right)$.

From [Vog84], it follows that ${ }^{n} M_{\overline{\mathfrak{p}}\left(\mathbf{c}^{\tau}\right)}\left(\xi\left(\mathbf{h}^{\tau}\right)\right)$ is irreducible for $\tau \in \Xi(\mathbf{u}, h, \mathbf{v})$. Hence, we see that $J(\mathbf{u}, h, \mathbf{v})$ is a primitive ideal.

We now discuss $\theta$-stable parabolic subalgebras with respect to $G$ (cf. [Vog97, Example 4.5]). Let $\ell$ be a positive integer. Let $\mathbb{P}_{\ell}(m, n)$ denote the set

$$
\left\{\left(\left(m_{1}, \ldots, m_{\ell}\right),\left(n_{1}, \ldots, n_{\ell}\right)\right) \in \mathbb{N}^{\ell} \times \mathbb{N}^{\ell} \mid \sum_{i=1}^{\ell} m_{i}=m, \sum_{i=1}^{\ell} n_{i}=n, \text { and } m_{j}+n_{j}>0 \text { for all } 1 \leqslant j \leqslant \ell\right\} .
$$

We also put $\mathbb{P}(m, n)=\bigcup_{\ell>0} \mathbb{P}_{\ell}(m, n)$ and $\mathbb{P}(0,0)=\mathbb{P}_{0}(0,0)=\{(\emptyset, \emptyset)\}$. If $(\mathbf{m}, \mathbf{n}) \in \mathbb{P}(m, n)$ satisfies $(\mathbf{m}, \mathbf{n}) \in \mathbb{P}_{\ell}(m, n)$, we call $\ell$ the length of $(\mathbf{m}, \mathbf{n})$. For $(\mathbf{m}, \mathbf{n}) \in \mathbb{P}(m, n)$, we define

$$
I_{(\mathbf{m}, \mathbf{n})}=\operatorname{diag}\left(I_{m_{1}},-I_{n_{1}}, \ldots, I_{m_{\ell}},-I_{n_{\ell}}\right)
$$

Here, for a positive integer $I_{k}$ means the $k \times k$ identity matrix. (If $k=0$, we simply ignore $I_{0}$.) Then we can consider the following realization of $G$,

$$
G=\left\{\left.g \in \mathrm{GL}(m+n, \mathbb{C})\right|^{\mathrm{t}} \bar{g} I_{(\mathbf{m}, \mathbf{n})} g=I_{(\mathbf{m}, \mathbf{n})}\right\} .
$$

Let $\theta$ be the Cartan involution given by conjugation by $I_{(\mathbf{m}, \mathbf{n})}$. In this realization, we let $\mathfrak{q}(\mathbf{m}, \mathbf{n})$ denote the block-upper-triangular parabolic subalgebra of $\mathfrak{g}=\mathfrak{g l}(m+n, \mathbb{C})$ with blocks of sizes $p_{1}+q_{1}, \ldots, p_{\ell}+q_{\ell}$ along the diagonal. Then, $\mathfrak{q}(\mathbf{m}, \mathbf{n})$ is a $\theta$-stable parabolic subalgebra. The corresponding Levi subgroup $\mathrm{U}(\mathbf{m}, \mathbf{n})$ consists of diagonal blocks,

$$
\mathrm{U}(\mathbf{m}, \mathbf{n}) \cong \mathrm{U}\left(m_{1}, n_{1}\right) \times \cdots \times \mathrm{U}\left(m_{\ell}, n_{\ell}\right) .
$$

We let $\mathfrak{g}(\mathbf{m}, \mathbf{n})$ denote the complexified Lie algebra of $U(\mathbf{m}, \mathbf{n})$, and write $\mathfrak{v}(\mathbf{m}, \mathbf{n})$ for the nilradical of $\mathfrak{q}(\mathbf{m}, \mathbf{n})$.

Via the above construction of $\mathfrak{q}(\mathbf{m}, \mathbf{n}), K_{\mathbb{C}}$-conjugacy classes of $\theta$-stable parabolic subalgebras of $G$ are parametrized by $\mathbb{P}(m, n)$.

Let $(\mathbf{m}, \mathbf{n})=\left(\left(m_{1}, \ldots, m_{\ell}\right),\left(n_{1}, \ldots, n_{\ell}\right)\right) \in \mathbb{P}_{\ell}(m, n)$ and $\mathbf{h}=\left(h_{1}, \ldots, h_{\ell}\right) \in \mathbb{Z}^{\ell}$. We define $\left(\eta_{\mathbf{h}}, \mathbb{C}_{\mathbf{h}}\right)$ to be the one-dimensional representation of $\mathbf{U}(\mathbf{m}, \mathbf{n})$ which restricts to $\mathbb{C}_{h_{i}}^{m_{i}, n_{i}}$ on each $\mathrm{U}\left(m_{i}, n_{i}\right)$ factor. We let $\mathcal{A}_{(\mathbf{m}, \mathbf{n})}[\mathbf{h}]$ denote the derived functor module

$$
A_{\mathfrak{q}(\mathbf{m}, \mathbf{n})}\left(\eta_{\mathbf{h}}\right) .
$$

Recall the terminology of the good, weakly fair, and mediocre ranges. (The first two are standard, e.g. [KV95, Introduction], the third is introduced in [Tra01].) A simple check shows that $\mathcal{A}_{(\mathbf{m}, \mathbf{n})}[\mathbf{h}$ ] is in the good range for $\mathfrak{q}(\mathbf{m}, \mathbf{n})$ if and only if $h_{i} \geqslant h_{i+1}$ for all $1 \leqslant i<\ell$, and $\mathcal{A}_{(\mathbf{m}, \mathbf{n})}[\mathbf{h}]$ is a derived functor module in the weakly fair range for $\mathfrak{q}(\mathbf{m}, \mathbf{n})$ if and only if

$$
h_{i}-h_{i+1} \geqslant-\frac{m_{i}+n_{i}+m_{i+1}+n_{i+1}}{2} \quad(1 \leqslant i<\ell) .
$$

For $(\mathbf{m}, \mathbf{n}) \in \mathbb{P}(m, n)$,

$$
\operatorname{Dim}\left(\mathcal{A}_{\mathbf{m}, \mathbf{n}}[\mathbf{h}]\right) \leqslant \frac{1}{2} \operatorname{dim} \mathcal{O}(\mathbf{m}, \mathbf{n}) .
$$

We call $(\mathbf{m}, \mathbf{n}) \in \mathbb{P}(m, n)$ normal if

$$
\operatorname{Dim}\left(\mathcal{A}_{\mathbf{m}, \mathbf{n}}[\mathbf{h}]\right)=\frac{1}{2} \operatorname{dim} \mathcal{O}(\mathbf{m}, \mathbf{n})
$$




\section{H. Matumoto and P. E. Trapa}

holds in the good range. It is known that for a normal $(\mathbf{m}, \mathbf{n}) \in \mathbb{P}_{\ell}(m, n)$ and any mediocre $\mathbf{h} \in \mathbb{Z}^{\ell}$, we have

$$
\operatorname{Dim}\left(\mathcal{A}_{\mathbf{m}, \mathbf{n}}[\mathbf{h}]\right)=\frac{1}{2} \operatorname{dim} \mathcal{O}(\mathbf{m}, \mathbf{n}) \quad \text { and } \quad \operatorname{AV}\left(\mathcal{A}_{\mathbf{m}, \mathbf{n}}[\mathbf{h}]\right)=\operatorname{AV}\left(\mathcal{A}_{\mathbf{m}, \mathbf{n}}\left[\mathbf{h}^{\prime}\right]\right),
$$

where $\mathbf{h}^{\prime}$ is any parameter in the good range (cf. [Tra01]).

Fix a sequence of positive integers $\mathbf{c}=\left(c_{1}, \ldots, c_{\ell}\right)$ such that $c_{1}+\cdots+c_{\ell}=m+n$. Put

$$
\mathbb{O}(\mathbf{c})=\left\{(\mathbf{m}, \mathbf{n}) \in \mathbb{P}_{\ell}(m, n) \mid(\mathbf{m}, \mathbf{n}) \text { is normal and } m_{i}+n_{i}=c_{i} \text { for all } 1 \leqslant i \leqslant \ell\right\}
$$

This set is of importance when determining the irreducible constituents of degenerate principal series of maximal Gelfand-Kirillov dimension. We take that up now.

THEOREM 7.3. In the notation of this section, consider a degenerate principal series representation of the form $\mathcal{I}_{P}[\mathbf{u} ; h ; \mathbf{v}]$ whose infinitesimal character is a weight-lattice translate of the infinitesimal character of the trivial representation. Define $\mathbf{c}$ and $\mathbf{h}$ from $(\mathbf{u}, h, \mathbf{v})$ as above. For $\tau \in \Xi(\mathbf{u}, h, \mathbf{v})$ (and using the notation at the end of $\S 2.4$ ), we have

$$
\mathcal{I}_{P}[\mathbf{u} ; h ; \mathbf{v}] \approx \bigoplus_{(\mathbf{m}, \mathbf{n}) \in \mathbb{O}\left(\mathbf{c}^{\tau}\right)} \mathcal{A}_{(\mathbf{m}, \mathbf{n})}\left[\check{\mathbf{h}}^{\tau}\right]
$$

here $\check{\mathbf{h}}^{\tau}=\left(\check{h}_{1}^{\tau}, \ldots, \check{h}_{2 s+1}^{\tau}\right)$ is defined by

$$
\check{h}_{i}^{\tau}=h_{\tau(i)}-\frac{m+n-c_{\tau(i)}}{2}+c_{i-1}^{\tau *} \quad \text { for } 1 \leqslant i \leqslant 2 s+1,
$$

where

$$
c_{i-1}^{\tau *}=c_{\tau(1)}+\cdots+c_{\tau(i-1)} \quad \text { for } 2 \leqslant i \leqslant 2 s+1,
$$

and $c_{0}^{\tau *}=0$.

Proof. Applying the translation principle into the weakly fair range [Vog84, Vog88], we may assume that $h_{\tau(1)} \gg \cdots \gg h_{\tau(2 s+1)}$.

For simplicity, we say that an infinitesimal character is $\rho$-integral if it is a weight lattice translate of the infinitesimal character of the trivial representation. From [BV83] (see also [Tra01, §6]) it follows that the item below holds.

(*) If $J$ is a primitive ideal with $\rho$-integral infinitesimal character, and $\mathcal{O}_{K} \in \operatorname{Irr}(\mathcal{O} \cap \mathfrak{s})$ where $\mathcal{O}$ is dense in the associated variety of $J$, then there is a unique irreducible Harish-Chandra module $A\left(J, \mathcal{O}_{K}\right)$ whose annihilator is $J$ and whose associated variety is the closure of $\mathcal{O}_{K}$.

Now let $\mathcal{O}$ denote the Richardson orbit associated to $\mathfrak{p}$. From the discussion, $J(\mathbf{u}, h, \mathbf{v})$ is primitive and its associated variety is the closure of $\mathcal{O}$. According to the definitions it follows that every constituent of maximal Gelfand-Kirillov dimension in $\mathcal{I}_{P}[\mathbf{u} ; h ; \mathbf{v}]$ is annihilated by $J(\mathbf{u}, h, \mathbf{v})$. Thus, if we set $J=J(\mathbf{u}, h, \mathbf{v})$ and write the associated cycle of $\mathcal{I}_{P}[\mathbf{u} ; h ; \mathbf{v}]$ according to Proposition 3.3(1) as

$$
\sum_{\mathcal{O}_{K}^{j} \in \operatorname{Irr}(\mathcal{O} \cap \mathfrak{s})}\left[\mathcal{O}_{K}^{j}\right]
$$

item $(*)$ and the additivity of the associated cycle on constituents of maximal Gelfand-Kirillov dimension immediately imply that

$$
\mathcal{I}_{P}[\mathbf{u} ; h ; \mathbf{v}] \approx \bigoplus_{\mathcal{O}_{K}^{j} \in \operatorname{Irr}(\mathcal{O} \cap \mathfrak{s})} A\left(J, \mathcal{O}_{K}^{j}\right) .
$$

From [Vog86, Proposition 16.8], we conclude that the annihilator of $\mathcal{A}_{(\mathbf{m}, \mathbf{n})}\left[\check{\mathbf{h}}^{\tau}\right]$ contains the annihilator of ${ }^{n} M_{\overline{\mathfrak{p}}\left(\mathbf{c}^{\tau}\right)}\left(\xi\left(\mathbf{h}^{\tau}\right)\right)$. Since $\operatorname{Dim}\left(\mathcal{A}_{(\mathbf{m}, \mathbf{n})}\left[\check{\mathbf{h}}^{\tau}\right]\right)=\frac{1}{2} \operatorname{dim} \mathcal{O}$, Proposition 7.2 implies that 


\section{LARGE IRREDUCIBLE CONSTITUENTS OF DEGENERATE PRINCIPAL SERIES}

the annihilator of $\mathcal{A}_{(\mathbf{m}, \mathbf{n})}\left[\check{\mathbf{h}}^{\tau}\right]$ coincides with $J$. On the other hand, it is easy to check (using the algorithm of $[$ Tra01, §5], for instance) that

$$
\left\{\operatorname{AV}\left(\mathcal{A}_{(\mathbf{m}, \mathbf{n})}\left[\check{\mathbf{h}}^{\tau}\right]\right) \mid(\mathbf{m}, \mathbf{n}) \in \mathbb{O}\left(\mathbf{c}^{\tau}\right)\right\}=\operatorname{Irr}(\mathcal{O} \cap \mathfrak{s}) .
$$

Thus,

$$
\bigoplus_{\mathcal{O}_{K}^{j} \in \operatorname{Irr}(\mathcal{O} \cap \mathfrak{s})} A\left(J, \mathcal{O}_{K}^{j}\right)=\bigoplus_{(\mathbf{m}, \mathbf{n}) \in \mathbb{O}\left(\mathbf{c}^{\tau}\right)} \mathcal{A}_{(\mathbf{m}, \mathbf{n})}\left[\check{\mathbf{h}}^{\tau}\right],
$$

and the theorem now follows from (7.2).

Next, we consider the case of the $P$-weakly fair range. (The terminology ' $P$-weakly fair' is introduced just before Proposition 6.13.) In that case, we have a result analogous to Theorem 6.18: the socle of the degenerate principal series is just the direct sum of the irreducible constituents of maximal Gelfand-Kirillov dimension and, moreover, each constituent corresponds to an open orbit in the complexified generalized flag manifold. The following result is proved in the same way as Theorem 6.18 .

Theorem 7.4. Assume $\mathbf{u}=\left(u_{1}, \ldots, u_{s}\right), \mathbf{v}=\left(v_{1}, \ldots, v_{s}\right) \in \mathbb{Z}^{s}$, and $h \in \mathbb{Z}$ satisfy

$$
u_{1} \geqslant \cdots \geqslant u_{s} \geqslant h \geqslant v_{s} \geqslant \cdots \geqslant v_{1} .
$$

Set

$$
\mathbf{c}(m, n, \kappa)=\left(k_{1}, \ldots, k_{s}, m+n-2 k_{s}^{*}, k_{s}, \ldots, k_{1}\right) \in \mathbb{N}^{2 s+1} .
$$

Let $\delta_{m, n}^{\kappa}$ denote the element of $\mathbb{C}^{s}$ whose the $i$ th entry is $\left(m+n-k_{i-1}^{*}\right) / 2$, where $k_{i}^{*}=k_{1}+\cdots+k_{i}$.

(1) We have

$$
\operatorname{Socle}\left(\mathcal{I}_{P}\left[\mathbf{u}+\delta_{m, n}^{\kappa} ; h ; \mathbf{v}-\delta_{m, n}^{\kappa}\right]\right) \cong \bigoplus_{(\mathbf{m}, \mathbf{n}) \in \mathbb{O}(\mathbf{c}(m, n ; \kappa))} \mathcal{A}_{(\mathbf{m}, \mathbf{n})}\left[u_{1}, \ldots, u_{s}, h, v_{s}, \ldots, v_{1}\right] .
$$

(2) There exists an embedding of generalized Verma modules

$$
{ }^{n} M_{\mathfrak{p}_{\kappa}}\left(-\xi\left(\mathbf{u}+\delta_{m, n}^{\kappa}, h, \mathbf{v}-\delta_{m, n}^{\kappa}\right)\right) \hookrightarrow{ }^{n} M_{\mathfrak{p}_{\kappa}}\left(-\xi\left(\mathbf{v}-\delta_{m, n}^{\kappa}, h, \mathbf{u}+\delta_{m, n}^{\kappa}\right)\right) .
$$

which induces an intertwining operator

$$
\varphi: \mathcal{I}_{P}\left[\mathbf{v}-\delta_{m, n}^{\kappa} ; h ; \mathbf{u}+\delta_{m, n}^{\kappa}\right] \rightarrow \mathcal{I}_{P}\left[\mathbf{u}+\delta_{m, n}^{\kappa} ; h ; \mathbf{v}-\delta_{m, n}^{\kappa}\right]
$$

Moreover,

$$
\operatorname{Image}(\varphi)=\operatorname{Socle}\left(\mathcal{I}_{P}\left[\mathbf{u}+\delta_{m, n}^{\kappa} ; h ; \mathbf{v}-\delta_{m, n}^{\kappa}\right]\right) .
$$

Since $\mathrm{U}(m, n)$ has a compact Cartan subgroup, Proposition 4.4 implies that $K_{\mathbb{C} \text {-conjugacy classes }}$ of $\theta$-stable parabolic subalgebras $\mathfrak{q}=\mathfrak{l}+\mathfrak{u}$ parametrize the open $G$-orbits on the complexified generalized flag variety $X_{P}=G_{\mathbb{C}} / P_{\mathbb{C}}$. Let $\chi$ be a holomorphic character of $M_{\mathbb{C}}$. We let $\mathcal{L}_{\chi}\left(\right.$ respectively, $\left.\omega_{P}\right)$ denote the holomorphic line bundle on $X_{P}$ associated to $\chi$ (respectively, the canonical line bundle on $\left.X_{P}\right)$. For an open $G$-orbit $\mathcal{V}$ in $X_{P}$, we consider the associated derived functor module

$$
A_{\mathcal{V}}(\chi)=H^{\operatorname{dim}(\mathfrak{k} \cap \mathfrak{u})}\left(\mathcal{V}, \mathcal{L}_{\chi} \otimes \omega_{P}\right)_{K \text {-finite }}
$$

We may thus rewrite Theorem 7.4 as follows.

Corollary 7.5. Recall the definition of the $P$-weakly fair range given before Proposition 6.13. For each $P$-weakly fair holomorphic character $\chi$ of $M_{\mathbb{C}}$, we have

$$
\operatorname{Socle}\left({ }^{n} \operatorname{Ind}_{P}^{G}\left(\mathbb{C}_{\delta_{P}} \otimes \chi\right)\right) \cong \bigoplus \mathcal{A}_{\mathcal{V}}(\chi),
$$

where the sum is taken over all good open $G$-orbits in $X_{P}$ (Definition 4.6). 


\section{H. Matumoto and P. E. Trapa}

\section{The group $\mathrm{GL}(n, \mathbb{H})$}

We turn to $G=\operatorname{GL}(n, \mathbb{H})$ and consider a parabolic subgroup $P=M N$ of $G$ with

$$
M \simeq \mathrm{GL}\left(k_{1}, \mathbb{H}\right) \times \cdots \times \mathrm{GL}\left(k_{s}, \mathbb{H}\right) .
$$

Here, $k_{1}+\cdots+k_{s}=n$. Write $\mathcal{O}$ for the Richardson orbit induced from the zero orbit of $\mathfrak{m}$. In this case $\operatorname{Irr}(\mathcal{O} \cap \mathfrak{s})$ consists of a single $K_{\mathbb{C}}$ orbit $\mathcal{O}_{K}$. Define

$$
\mathcal{I}(\mathcal{O})={ }^{n} \operatorname{Ind}_{P}^{G}(\mathbb{1}) .
$$

This is unitarily induced and it follows from $[\operatorname{Vog} 86]$ that $\mathcal{I}(\mathcal{O})$ is irreducible. The associated variety of $\mathcal{I}(\mathcal{O})$ is $\overline{\mathcal{O}_{K}^{1}}$ (as can be deduced from Theorem 3.1, for instance) and it has the special unipotent infinitesimal character associated to $\mathcal{O}(\mathrm{cf}$. [BV85]). Thus, the results of [BV85] imply that $\mathcal{I}(\mathcal{O})$ is annihilated by $\mathrm{J}^{\max }(\lambda(\mathcal{O}))$.

The structure of the Harish-Chandra cells for $\mathrm{GL}(n, \mathbb{H})$ is easier than that for $\operatorname{Sp}(p, q)$ and $\mathrm{SO}^{*}(2 n)$ (see, e.g., [McG98]). From an argument similar to that leading to Theorem 6.12, we obtain the following result.

Theorem 8.1. We have that $\mathcal{I}(\mathcal{O})$ is the unique weakly unipotent representation attached to $\mathcal{O}$ with integral infinitesimal character.

On the other hand, for $G=\mathrm{GL}(n, \mathbb{H})$ the unique open $G$-orbit in the complexified flag manifold $X_{P}=G_{\mathbb{C}} / P_{\mathbb{C}}$ is not necessarily good. In fact, we have the following result.

Proposition 8.2. The unique open $G$-orbit in $X_{P}$ (say $\mathcal{V}$ ) is good (Definition 4.6) if and only if $P$ is $G$-conjugate to its opposite parabolic subgroup.

Proof. First, we remark it is obvious that $\mathfrak{p}$ is neat in the sense of Definition 4.5 if and only if $P$ is $G$-conjugate to its opposite parabolic subgroup. So the if part of the current proposition follows from Lemma 4.3. The only-if part is proved as follows. Let $\mathcal{C}$ be the unique $K_{\mathbb{C}}$ orbit contained in $\mathcal{V}$. Let $\mathfrak{q}$ be any parabolic subalgebra contained in $\mathcal{C}$. Then, from Lemma 4.3, there is $\theta$-stable Borel subalgebra $\mathfrak{b}$ such that $\mathfrak{b} \subseteq \mathfrak{q}$. We choose a $\theta$-stable Cartan subalgebra $\mathfrak{h}$ such that $\mathfrak{h} \subseteq \mathfrak{b}$. If we consider the basis of the root system $\Delta(\mathfrak{g}, \mathfrak{h})$ corresponding to $\mathfrak{b}, \theta$ induces the nontrivial automorphism of the Dynkin diagram. Hence, we see that $\theta(\mathfrak{q})$ is $\operatorname{Ad}\left(G_{\mathbb{C}}\right)$-conjugate to the opposite parabolic subalgebra. So, $\mathfrak{p}$ is $\operatorname{Ad}\left(G_{\mathbb{C}}\right)$-conjugate to the opposite parabolic subalgebra. The theorem follows.

We have the following analog of Theorem 6.12.

Theorem 8.3. Let $P$ be a parabolic subgroup of $G=\mathrm{GL}(n, \mathbb{H})$ such that $P$ is $G$-conjugate to its opposite parabolic subgroup. Let $\mathfrak{q}=\mathfrak{l}+\mathfrak{u}$ be a $\theta$-stable parabolic subalgebra in the unique closed

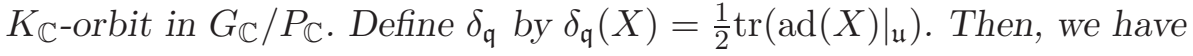

$$
\mathcal{I}(\mathcal{O}) \cong A_{\mathfrak{q}}\left(-\delta_{\mathfrak{q}}\right) \text {. }
$$

(This is a derived functor module on the 'edge' of the weakly fair range in the sense of Remark 6.3.)

Proof. One may verify that $A_{\mathfrak{q}}\left(-\delta_{\mathfrak{q}}\right)$ is irreducible and integrally weakly unipotent attached to $\mathcal{O}$. So the result follows from Theorem 8.1.

As in the case of $\operatorname{Sp}(p, q)$ and $\mathrm{SO}^{*}(2 n)$, we have the following result which is deduced in the same way as Corollary 6.15.

Corollary 8.4. Let $P$ be a parabolic subgroup of $G=\mathrm{GL}(n, \mathbb{H})$ and let $X$ be a representation of $G$ parabolically induced from a one-dimensional representation of $P$. Assume that $P$ is $G$-conjugate to 


\section{LARGE IRREDUCIBLE CONSTITUENTS OF DEGENERATE PRINCIPAL SERIES}

its opposite parabolic subgroup. Moreover, assume that $X$ has integral infinitesimal character. Then any irreducible constituent of $X$ of the maximal Gelfand-Kirillov dimension is a derived functor module in the weakly fair range.

We define $\mathcal{I}_{P}(\chi)$ and $A_{\mathcal{V}}(\chi)$ in the same way as the case of $\operatorname{Sp}(p, q)$ and $\mathrm{SO}^{*}(2 n)$. Since every involution in the Weyl group of the type A is a Duflo involution, [Mat93, Proposition 2.1.2] implies the following result.

Theorem 8.5. Let $G=\mathrm{GL}(n, \mathbb{H})$ and assume that the parabolic subgroup $P=M N$ is $G$-conjugate to its opposite. Let $\chi$ be a $P$-weakly fair holomorphic character of $M_{\mathbb{C}}$. Then we have the following.

(a) There is an embedding of generalized Verma module

$$
\psi_{\chi}:{ }^{u} M_{\mathfrak{p}}\left(-2 \delta_{P}-d \chi\right) \hookrightarrow{ }^{u} M_{\mathfrak{p}}(d \chi)
$$

which induces an intertwining operator

$$
\Psi_{\chi}: \mathcal{I}_{P}\left(\chi^{-1} \otimes \xi_{-2 \delta_{P}}\right) \rightarrow \mathcal{I}_{P}(\chi) .
$$

(b) We have $\operatorname{Socle}\left(\mathcal{I}_{P}(\chi)\right)=\Psi_{\chi}\left(\mathcal{I}_{P}\left(\chi^{-1} \otimes \xi_{-2 \delta_{P}}\right)\right)$ and, moreover,

$$
\operatorname{Socle}\left(\mathcal{I}_{P}(\chi)\right) \approx \mathcal{I}_{P}(\chi) \text {. }
$$

(c) We have

$$
\operatorname{Socle}\left(\mathcal{I}_{P}(\chi)\right) \cong A_{\mathcal{V}}(\chi)
$$

\section{Complex groups}

Let $G$ be a connected complex reductive Lie group. Let $\mathfrak{g}$ be the Lie algebra of $G, \mathrm{U}(\mathfrak{g})$ the universal enveloping algebra of $\mathfrak{g}, \mathfrak{h}$ a Cartan subalgebra of $\mathfrak{g}$, and $\Delta$ the root system with respect to $(\mathfrak{g}, \mathfrak{h})$. Let $W$ be the Weyl group of the pair $(\mathfrak{g}, \mathfrak{h})$ and let $w_{0}$ denote the longest element of $W$. Fix a positive system $\Delta^{+}$of $\Delta$ and let $\Pi$ denote the corresponding basis of $\Delta$. Fix a triangular decomposition $\mathfrak{g}=\overline{\mathfrak{n}} \oplus \mathfrak{h} \oplus \mathfrak{n}$ such that $\mathfrak{n}=\sum_{\alpha \in \Delta^{+}} \mathfrak{g}_{\alpha}$ and $\bar{n}=\sum_{-\alpha \in \Delta^{+}} \mathfrak{g}_{\alpha}$. Set $\mathfrak{b}=\mathfrak{h}+\mathfrak{n}$.

Let $\mathfrak{g}_{0}$ denote the normal real form of $\mathfrak{g}$ which is compatible with the above decomposition and let $X \leadsto \bar{X}$ denote the corresponding complex conjugation. Then there is an anti-automorphism of $\mathrm{U}(\mathfrak{g})$, the so-called Chevalley anti-automorphism, denoted $u \mapsto{ }^{\mathrm{t}} u$ which satisfies the following when restricted to $\mathfrak{g}$ :

(1) ${ }^{\mathrm{t}} \mathfrak{g}_{0}=\mathfrak{g}_{0}$;

(2) ${ }^{t} \mathfrak{n}=\overline{\mathfrak{n}},{ }^{\mathrm{t}} \overline{\mathfrak{n}}=\mathfrak{n}$;

(3) ${ }^{\mathrm{t}} X=X(X \in \mathfrak{h})$.

Define a homomorphism of real Lie algebras $\mathfrak{g} \rightarrow \mathfrak{g} \times \mathfrak{g}$ via $X \leadsto(X, \bar{X})$ for $X \in \mathfrak{g}$. Then the image of this homomorphism is a real form of $\mathfrak{g} \times \mathfrak{g}$, and so we can regard $\mathfrak{g} \times \mathfrak{g}$ as the complexification $\mathfrak{g}_{\mathbb{C}}$ of $\mathfrak{g}$. Then $\mathfrak{k}_{\mathbb{C}}:=\left\{\left(X,-{ }^{t} X\right) \mid X \in \mathfrak{g}\right\}$ is identified with the complexification of a compact form of $\mathfrak{g}$ and, of course, $\mathfrak{k}_{\mathbb{C}}$ is also identified (as a complex Lie algebra) with $\mathfrak{g}$ via $X \leadsto\left(X,-{ }^{\mathrm{t}} X\right)$. We may regard $G \times G$ as the complexification $G_{\mathbb{C}}$ of $G$.

Let $P$ be a standard parabolic subgroup of $G$; namely, assume that the Lie algebra $\mathfrak{p}$ of $P$ contains $\mathfrak{b}$. Let $M$ (respectively, $\mathfrak{m}$ ) denote the Levi part of $P$ (respectively, $\mathfrak{p}$ ) stable under the Chevalley anti-automorphism. Write $\mathfrak{n}_{\mathfrak{p}}$ for the nilradical of $\mathfrak{p}$. Let $S$ be the subset of $\Pi$ corresponding to $\mathfrak{p}$, i.e. $S$ is the basis of the root system of $\mathfrak{h}$ in $\mathfrak{m}$. Put $S^{\prime}=\left\{-w_{0} \alpha \mid \alpha \in S\right\}$. Let $\mathfrak{p}^{\prime}$ denote the standard parabolic subalgebra corresponding to $S^{\prime}$. Let $w_{\mathfrak{p}}$ denote the longest element of the Weyl group with respect to $(\mathfrak{m}, \mathfrak{h})$. 


\section{H. Matumoto and P. E. Trapa}

Under the above identification $G_{\mathbb{C}} \cong G \times G$, the complexification $P_{\mathbb{C}}$ of $P$ is identified with a subgroup $P \times P$ of $G \times G$, and the complex generalized flag variety $X=G_{\mathbb{C}} / P_{\mathbb{C}}$ is identified with $G / P \times G / P . X$ can be regarded as the set of parabolic subalgebras of $\mathfrak{g}$ which are $\operatorname{Ad}\left(G_{\mathbb{C}}\right)$-conjugate to $\operatorname{Lie}\left(P_{\mathbb{C}}\right)=\mathfrak{p}_{\mathbb{C}} \cong \mathfrak{p} \times \mathfrak{p}$.

The following is well known.

Proposition 9.1. We have that $X$ has a unique $G$-orbit (say $\mathcal{O}_{0}$ ). The following five conditions are equivalent to each other.

(1) $\mathcal{O}_{0}$ contains a $\theta$-stable parabolic subalgebra of $\mathfrak{g}_{\mathbb{C}}$;

(2) $\mathfrak{p}$ and the parabolic subalgebra opposite to $\mathfrak{p}$ are $G$-conjugate;

(3) $\mathfrak{p}=\mathfrak{p}^{\prime}$;

(4) $S$ is stable under the action of $-w_{0}$;

(5) $w_{0} w_{\mathfrak{p}}=w_{\mathfrak{p}} w_{0}$ (namely $w_{0} w_{\mathfrak{p}}$ is an involution).

For $X \in \mathfrak{m}$, define $\delta_{\mathfrak{p}}(X)=\frac{1}{2} \operatorname{tr}\left(\left.\operatorname{ad}_{\mathfrak{g}}(X)\right|_{\mathfrak{n}_{\mathfrak{p}}}\right)$. Then, $\delta_{\mathfrak{p}}$ is a one-dimensional representation of $\mathfrak{m}$, and $2 n \delta_{\mathfrak{p}}$ lifts to a holomorphic group homomorphism $\xi_{2 n \delta_{\mathfrak{p}}}: M \rightarrow \mathbb{C}^{\times}$for all $n \in \mathbb{Z}$. Identifying $M_{\mathbb{C}} \cong M \times M$, for $n, m \in 2 \mathbb{Z}$ we can regard $\chi_{n, m}=\xi_{n \delta_{\mathfrak{p}}} \otimes \xi_{m \delta_{\mathfrak{p}}}$ as a real analytic group homomorphism $M \rightarrow \mathbb{C}^{\times}$. Taking account of the natural projection $P \rightarrow M$, we regard $\chi_{n, m}$ as a real analytic one-dimensional representation of $P$. We may thus consider an unnormalized degenerate principal series representation,

$$
{ }^{u} \operatorname{Ind}_{P}^{G}\left(\chi_{n, m}\right)=\left\{f \in C^{\infty}(G) \mid f(g p)=\chi_{n, m}(p)^{-1} f(g)(g \in G, p \in P)\right\}_{\mathfrak{k}_{\mathbb{C}} \text {-finite }} .
$$

If $\mathfrak{p}=\mathfrak{b}$, then $\delta_{\mathfrak{b}}=\rho=\frac{1}{2} \sum_{\alpha \in \Delta^{+}} \alpha$. For $t \in \mathbb{R}$, we define an unnormalized generalized Verma module,

$$
{ }^{u} M_{\mathfrak{p}}(t)=\mathrm{U}(\mathfrak{g}) \otimes_{\mathrm{U}(\mathfrak{p})} \mathbb{C}_{t \delta_{\mathfrak{p}}} .
$$

Here, $\mathbb{C}_{t \delta_{\mathfrak{p}}}$ means the one-dimensional module of $\mathfrak{p}$ such that $\mathfrak{m}$ acts on it by $t \delta_{\mathfrak{p}}$. If $\mathfrak{p}=\mathfrak{b}$, we simply write ${ }^{u} M(t)={ }^{u} M_{\mathfrak{b}}(t)$. We let $I_{\mathfrak{p}}(t)$ denote the annihilator of $M_{\mathfrak{p}}(t)$ in $\mathrm{U}(\mathfrak{g})$. From [Vog84], $M_{\mathfrak{p}}(t)$ is irreducible for $t \leqslant-1$. Therefore, $I_{\mathfrak{p}}(t)$ is a primitive ideal for $t \leqslant-1$.

Under the identification $\mathfrak{g}_{\mathbb{C}} \cong \mathfrak{g} \times \mathfrak{g}$, we identify $\mathrm{U}\left(\mathfrak{g}_{\mathbb{C}}\right) \cong \mathrm{U}(\mathfrak{g}) \otimes \mathrm{U}(\mathfrak{g})$. We write $u \rightsquigarrow \check{u}(u \in \mathrm{U}(\mathfrak{g}))$ for the anti-automorphism of $\mathrm{U}(\mathfrak{g})$ generated by $X \leadsto-X(X \in \mathfrak{g})$. If $V$ is a $\mathrm{U}(\mathfrak{g})$-bimodule, we can regard $V$ as a $\mathrm{U}\left(\mathfrak{g}_{\mathbb{C}}\right)$-module as follows,

$$
\left(u_{1} \otimes u_{2}\right) v={ }^{\mathrm{t}} \check{u}_{1} v \check{u}_{2} \quad\left(u_{1}, u_{2} \in \mathrm{U}(\mathfrak{g}), v \in V\right) .
$$

In particular, we may regard $\mathrm{U}(\mathfrak{g}) / I_{\mathfrak{p}}(t)$ as a $\mathrm{U}\left(\mathfrak{g}_{\mathbb{C}}\right)$-module.

We quote the following.

Theorem 9.2 [CD77, Corollaire 2.12 and Corollaire 6.3]. If $n$ is an even integer such that $n \geqslant 2$, then ${ }^{u} \operatorname{Ind}_{P}^{G}\left(\chi_{n, n}\right)$ is isomorphic to $\mathrm{U}(\mathfrak{g}) / I_{\mathfrak{p}}(-n)$ as $\mathrm{U}\left(\mathfrak{g}_{\mathbb{C}}\right)$-modules.

Together with [BK76, Korollar 3.6] and the primitivity of $I_{\mathfrak{p}}(-n)(n \geqslant 0)$, we have the following.

Corollary 9.3. If $n \geqslant 2$, then ${ }^{u} \operatorname{Ind}_{P}^{G}\left(\chi_{n, n}\right)$ has a unique irreducible submodule (say $Y_{n}$ ). The Gelfand-Kirillov dimension of $Y_{n}$ is equal to that of ${ }^{u} \operatorname{Ind}_{P}^{G}\left(\chi_{n, n}\right)$. The Gelfand-Kirillov dimension of ${ }^{u} \operatorname{Ind}_{P}^{G}\left(\chi_{n, n}\right) / Y_{n}$ is strictly smaller than that of $Y_{n}$.

Since ${ }^{u} \operatorname{Ind}_{P}^{G}\left(\chi_{2,2}\right)$ is the induced module associated with the canonical bundle, we only consider the case of $n=2$. For simplicity, we put $I=I_{\mathfrak{p}}(-2)=I_{\mathfrak{p}^{\prime}}(0)$. (The latter equality follows from [BJ77, 4.10 Corollar] where is it proved that $I_{\mathfrak{p}}(-n)=I_{\mathfrak{p}^{\prime}}(n-2)$.) Let $M$ and $N$ be $\mathrm{U}(\mathfrak{g})$-modules. We know that $\operatorname{Hom}_{\mathbb{C}}(M, N)$ has a natural structure of a $\mathrm{U}(\mathfrak{g})$-bimodule (using the Chevalley anti-automorphism), and so we may consider $\operatorname{Hom}_{\mathbb{C}}(M, N)$ as a $\mathrm{U}\left(\mathfrak{g}_{\mathbb{C}}\right)$ module. 


\section{LARGE IRREDUCIBLE CONSTITUENTS OF DEGENERATE PRINCIPAL SERIES}

We denote the $\mathfrak{k}_{\mathbb{C}}$-finite part of $\operatorname{Hom}(M, N)$ by $L(M, N)$. The functor $V \rightsquigarrow L\left({ }^{u} M(0), V\right)$ defines an equivalence of categories between a category of highest weight modules and the category of Harish-Chandra $\left(\mathfrak{g}_{\mathbb{C}}, \mathfrak{k}_{\mathbb{C}}\right.$ )-modules (Bernstein-Gelfand-Joseph-Enright, cf. [BG80, Jos79]). Via this equivalence of categories, the two-sided ideals of $\mathrm{U}(\mathfrak{g}) / I$ correspond to the $\mathrm{U}(\mathfrak{g})$-submodules of ${ }^{u} M(0) / I^{u} M(0)$. In particular $Y_{2}$ corresponds to the irreducible highest weight module with the highest weight $\tau \rho-\rho$ (see [Jos79] and also [Jos83, p. 43]). Here $\tau \in W$ is the Duflo involution associated to the primitive ideal $I$. For integral weights $\mu, \nu \in \mathfrak{h}^{*}$, we let $V(\nu, \mu)$ denote the Langlands (Zhelobenko) subquotient of ${ }^{u} \operatorname{Ind}_{B}^{G}\left(\xi_{\nu+\rho} \otimes \xi_{\mu+\rho}\right)$. From [Jos79, Proposition 4.5], we have the following.

TheOREM 9.4. The unique irreducible submodule $Y_{2}$ of ${ }^{u} \operatorname{Ind}_{P}^{G}\left(\chi_{2,2}\right)$ is isomorphic to $V(-\tau \rho,-\rho)=$ $V\left(w_{0} \tau w_{0} \rho, \rho\right)$.

Using the equivalent conditions in Proposition 9.1, we may compare $Y_{2}$ with

$$
\mathcal{A}_{\mathcal{O}_{0}}=H^{\operatorname{dim} \mathfrak{n}_{\mathfrak{p}}}\left(\mathcal{O}_{0}, \mathcal{L}\right)_{\mathfrak{E}^{-} \text {-finite }}
$$

For orientation, we include the following result.

Theorem 9.5 [Enr79, VZ84]. Assume that $\mathfrak{p}$ satisfies the equivalent conditions in Proposition 9.1. Then we have

$$
\mathcal{A}_{\mathcal{O}_{0}} \cong{ }^{u} \operatorname{Ind}_{P}^{G}\left(\chi_{2,0}\right) \cong{ }^{u} \operatorname{Ind}_{P}^{G}\left(\chi_{0,2}\right) .
$$

Since $2 \delta_{\mathfrak{p}}=\rho-w_{0} w_{\mathfrak{p}} \rho$, we see that $\mathcal{A}_{\mathcal{O}_{0}}$ is the Langlands subquotient $V\left(-w_{0} w_{\mathfrak{p}} \rho,-\rho\right)$ of ${ }^{u} \operatorname{Ind}_{B}^{G}\left(\xi_{-w_{0} w_{\mathfrak{p}} \rho+\rho} \otimes \xi_{-\rho+\rho}\right)$. Since ${ }^{u} M_{\mathfrak{p}}(-2)$ is the irreducible highest weight module of the highest weight $-2 \delta_{\mathfrak{p}}=w_{0} w_{\mathfrak{p}} \rho-\rho$, we have the following.

Theorem 9.6. Assume that $\mathfrak{p}$ satisfies the equivalent conditions in Proposition 1.2(1). Then $\mathcal{A}_{\mathcal{O}_{0}}$ is isomorphic to the unique submodule of ${ }^{u} \operatorname{Ind}_{P}^{G}\left(\chi_{2,2}\right)$ if and only if $w_{0} w_{\mathfrak{p}}$ is a Duflo involution in $W$.

In the case that $w_{0} w_{\mathfrak{p}}$ is a Duflo involution, the embedding of $\mathcal{A}_{\mathcal{O}_{0}}$ into ${ }^{u} \operatorname{Ind}{ }_{P}^{G}\left(\chi_{2,2}\right)$ can be regarded as giving rise to intertwining operators between the following degenerate principal series representation:

$$
\begin{aligned}
{ }^{u} \operatorname{Ind}_{P}^{G}\left(\chi_{2,0}\right) & \hookrightarrow{ }^{u} \operatorname{Ind}_{P}^{G}\left(\chi_{2,2}\right), \\
{ }^{u} \operatorname{Ind}_{P}^{G}\left(\chi_{0,2}\right) & \hookrightarrow{ }^{u} \operatorname{Ind}_{P}^{G}\left(\chi_{2,2}\right) .
\end{aligned}
$$

In fact, the following result holds.

Proposition 9.7 [Mat93]. Let $t$ be a nonnegative even integer. Then we have

$$
{ }^{u} M_{\mathfrak{p}}(-t-2) \hookrightarrow{ }^{u} M_{\mathfrak{p}}(t)
$$

if and only if $w_{0} w_{\mathfrak{p}}$ is a Duflo involution in $W$.

Taking account of the isomorphism ${ }^{u} \operatorname{Ind}_{P}^{G}\left(\chi_{m, n}\right) \cong\left({ }^{u} M_{\mathfrak{p}}(-m) \otimes{ }^{u} M_{\mathfrak{p}}(-n)\right)_{\mathfrak{k}_{\mathbb{C}} \text {-finite }}^{*}$, the intertwining operators of (9.1) and (9.2) are induced from the embeddings the generalized Verma modules in the proposition.

Finally, if $W$ is a Weyl group of the type A, each involution in $W$ is a Duflo involution [Duf77]. Hence, we obtain the following.

Corollary 9.8. If $G=\mathrm{GL}(n, \mathbb{C})$ and $\mathfrak{p}$ satisfies the equivalent conditions in Proposition 1.1, the socle of ${ }^{u} \operatorname{Ind}_{P}^{G}\left(\chi_{2,2}\right)$ is isomorphic to $\mathcal{A}_{\mathcal{O}_{0}}$. 


\section{H. Matumoto and P. E. Trapa}

\section{ACKNOWLEDGEMENTS}

During the initial stages of this project, the authors participated in the Representation Theory of Lie Groups program at the Institute for Mathematical Sciences (IMS) at the National University of Singapore. It is our pleasure to thank the IMS for its warm hospitality. The first author is supported in part by Grant-in-Aid for Scientific Research (No. 50272597). The second author is supported by NSF grant DMS-0300106.

\section{REFERENCES}

Bar00 D. Barbasch, Orbital integrals of nilpotent orbits, in The mathematical legacy of Harish-Chandra, Proceedings of Symposia in Pure Mathematics, vol. 68 (American Mathematical Society, Providence, RI, 2000), 97-110.

BV80 D. Barbasch and D. A. Vogan Jr., The local structure of characters, J. Funct. Anal. 37 (1980), $27-55$.

BV83 D. Barbasch and D. A. Vogan Jr., Weyl group representations and nilpotent orbits, in Representation theory of reductive groups, Progress in Mathematics, vol. 40 (Birkhäuser, Boston, MA, 1983), 21-33.

BV85 D. Barbasch and D. A. Vogan Jr., Unipotent representations of complex semisimple Lie groups, Ann. of Math. (2) 121 (1985), 41-110.

BG80 J. N. Bernstein and S. I. Gelfand, Tensor products of finite-and infinite-dimensional representations of semisimple Lie algebras, Compositio Math. 41 (1980), 245-285.

BJ77 W. Borho and J. C. Jantzen, Über primitive Ideale in der Einhüllenden einer halbeinfachen Lie-Algebra, Invent. Math. 39 (1977), 1-53.

BK76 W. Borho and H. Kraft, Über die Gelfand-Kirillov Dimension, Math. Ann. 220 (1976), 1-24.

CM94 D. H. Collingwood and W. M. McGovern, Nilpotent orbits in semisimple Lie algebras (Chapman and Hall, London, 1994).

CS90 D. H. Collingwood and B. Shelton, A duality theorem for extensions of induced highest weight modules, Pacific J. Math. 146 (1990), 227-237.

CD77 N. Conze-Berline and M. Duflo, Sur les représentations induites des groupes semi-simples complexes, Compositio Math. 34 (1977), 307-336.

Duf77 M. Duflo, Sur la classification des idéau primitifs dans l'algèbre enveloppante d'une algèbre de Lie semi-simple, Ann. of Math. (2) 105 (1977), 107-120.

Enr79 T. J. Enright, Relative Lie algebra cohomology and unitary representations of complex Lie groups, Duke Math. J. 46 (1979), 513-525.

Gin93 S. Gindikin, Holomorphic language for $\bar{\partial}$-cohomology and representations of real semisimple Lie groups, in The Penrose transform and analytic cohomology in representation theory, South Hadley, MA, 1992, Contemporary Mathematics, vol. 154 (American Mathematical Society, Providence, RI, 1993), 103-115.

Hes78 W. Hesselink, Polarizations in classical groups, Math. Z. 160 (1978), 217-234.

Jos79 A. Joseph, Dixmier's problem for Verma and principal series submodules, J. London Math. Soc. (2) 20 (1979), 193-204.

Jos83 A. Joseph, On the classification of primitive ideals in the enveloping algebra of a semisimple Lie algebra, Lecture Notes in Mathematics, vol. 1024 (Springer, Berlin, 1983), 103-115.

Jos85 A. Joseph, On the associated variety of a primitive ideal, J. Algebra 93 (1985), 509-523.

KL83 M. Kashiwara and Y. Laurent, Théorèmes d'annulation et deuxième microlocalisation (Université de Paris-Sud, Paris 1983).

KV95 A. W. Knapp and D. A. Vogan Jr., Cohomological induction and unitary representations, Princeton Mathematical Series, vol. 45 (Princeton University Press, Princeton, NJ, 1995).

Kos61 B. Kostant, Lie algebra cohomology and the generalized Borel-Weil theorem, Ann. of Math. (2) 74 (1961), 329-387. 


\section{LARGE IRREDUCIBLE CONSTITUENTS OF DEGENERATE PRINCIPAL SERIES}

LX88 G. Lusztig and N. Xi, Canonical left cells in affine Weyl groups, Adv. Math. 72 (1988), 284-288.

Mat79 T. Matsuki, The orbits of affine symmetric spaces under the action of minimal parabolic subgroups, J. Math. Soc. Japan 31 (1979), 331-357.

Mat88a T. Matsuki, Closure relations for orbits on affine symmetric spaces under the action of parabolic subgroups, Hiroshima Math. J. 18 (1988), 59-67.

Mat88b H. Matumoto, Cohomological Hardy space for $\mathrm{SU}(2,2)$, in Representations of Lie groups, Kyoto, Hiroshima, 1986, Advanced Studies in Pure Mathematics, vol. 14 (Academic Press, Boston, MA, 1988), 469-497.

Mat93 H. Matumoto, On the existence of homomorphisms between scalar generalized Verma modules, Contemporary Mathematics, vol. 145 (American Mathematical Society, Providence, RI, 1993), 259-274.

Mat96 H. Matumoto, On the representations of $\mathrm{U}(m, n)$ unitarily induced from derived functor modules, Compositio Math. 100 (1996), 1-39.

Mat03 H. Matumoto, The homomorphisms between scalar generalized Verma modules associated to maximal parabolic subalgebras, Duke Math. J. 131 (2006), 75-118.

Mat04 H. Matumoto, On the representations of $\mathrm{Sp}(p, q)$ and $\mathrm{SO}^{*}(2 n)$ unitarily induced from derived functor modules, Composito Math. 140 (2004), 1059-1096.

McG98 W. M. McGovern, Cells of Harish-Chandra modules for real classical groups, Amer. J. Math. 120 (1998), 211-228.

PT02 A. Paul and P. E. Trapa, One-dimensional representations of $\mathrm{U}(p, q)$ and the Howe correspondence, J. Funct. Anal. 195 (2002), 129-166.

Sah92 S. Sahi, The Capelli identity and unitary representations, Compositio Math. 81 (1992), 247-260.

Sah95 S. Sahi, Jordan algebras and degenerate principal series, J. reine angew. Math. 462 (1995), 1-18.

SS90 S. Sahi and E. M. Stein, Analysis in matrix space and Speh's representations, Invent. Math. 101 (1990), 379-393.

SV00 W. Schmid and K. Vilonen, Characteristic cycles and wave front cycles of representations of reductive Lie groups, Ann. of Math. (2) 151 (2000), 1071-1118.

Tra01 P. E. Trapa, Annihilators and associated varieties of $A_{\mathfrak{q}}(\lambda)$ modules for $\mathrm{U}(p, q)$, Compositio Math. 129 (2001), 1-45.

Tra05 P. E. Trapa, Richardson orbits for real Lie groups, J. Algebra 286 (2005), 361-385.

Vog78 D. A. Vogan Jr., Gelfand-Kirillov dimension of Harish-Chandra modules, Invent. Math. 48 (1978), 75-98.

Vog84 D. A. Vogan Jr., Unitarizability of certain series of representations, Ann. of Math. (2) 120 (1984), 141-187.

Vog86 D. A. Vogan Jr., The unitary dual of GL(n) over an archimedean field, Invent. Math. 83 (1986), 449-505.

Vog87 D. A. Vogan Jr., Unitary representations of reductive Lie groups, Annals of Mathematics Studies, vol. 118 (Princeton University Press, Princeton, NJ, 1987).

Vog88 D. A. Vogan Jr., Irreducibility of discrete series representations for semisimple symmetric spaces, in Representations of Lie groups, Kyoto, Hiroshima, 1986, Advanced Studies in Pure Mathematics, vol. 14 (Academic Press, Boston, MA, 1988), 191-221.

Vog90 D. A. Vogan Jr., Dixmier algebras, sheets, and representation theory, in Operator algebras, unitary representations, enveloping algebras, and invariant theory, Paris, 1989, Progress in Mathematics, vol. 92 (Birkhäuser, Boston, MA, 1990), 333-395.

Vog91 D. A. Vogan Jr., Associated varieties and unipotent representations, in Proceedings of the Bowdoin Conference on Harmonic Analysis, Progress in Mathematics, vol. 101 (Birkhäuser, Boston, MA, 1991).

Vog97 D. A. Vogan Jr., Cohomology and group representations, in Representation theory and automorphic forms, Edinburgh, 1996, Proceedings of Symposia in Pure Mathematics, vol. 61 (American Mathematical Society, Providence, RI, 1997), 219-243. 


\section{LARGE IRREDUCIBLE CONSTITUENTS OF DEGENERATE PRINCIPAL SERIES}

VZ84 D. A. Vogan Jr. and G. Zuckerman, Unitary representations with non-zero cohomology, Compositio Math. 53 (1984), 51-90.

Wal03 N. R. Wallach, Generalized Whittaker vectors for holomorphic and quaternionic representations, Comment. Math. Helv. 78 (2003), 266-307.

Won93 H. Wong, Dolbeault cohomologies and Zuckerman modules, in The Penrose transform and analytic cohomology in representation theory, South Hadley, MA, 1992, Contemporary Mathematics, vol. 154 (American Mathematical Society, Providence, RI, 1993), 217-223.

Yam97 A. Yamamoto, Orbits in the flag variety and images of the moment map for classical groups. I, Represent. Theory 1 (1997), 329-404.

Zha95 G. K. Zhang, Jordan algebras and generalized principal series representations, Math. Ann. 302 (1995), 773-786.

Zuc77 G. Zuckerman, Tensor products of finite and infinite dimensional representations of semisimple Lie groups, Ann. of Math. (2) 106 (1977), 295-308.

Hisayosi Matumoto hisayosi@ms.u-tokyo.ac.jp

Graduate School of Mathematical Sciences, University of Tokyo, 3-8-1 Komaba Meguro-ku, Tokyo 153-8902, Japan

Peter E. Trapa ptrapa@math.utah.edu

Department of Mathematics, University of Utah, Salt Lake City, UT 84112, USA 